WAPD-TM-205

AEC RESEARCH AND

DEVELOPMENT REPORT

\title{
EURIPUS-3 AND \\ DAEDALUS--MONTE CARLO \\ DENSITY \\ CODES FOR THE \\ IBM-704
}

February 1960

CONTRACT AT-11-1-GEN-14

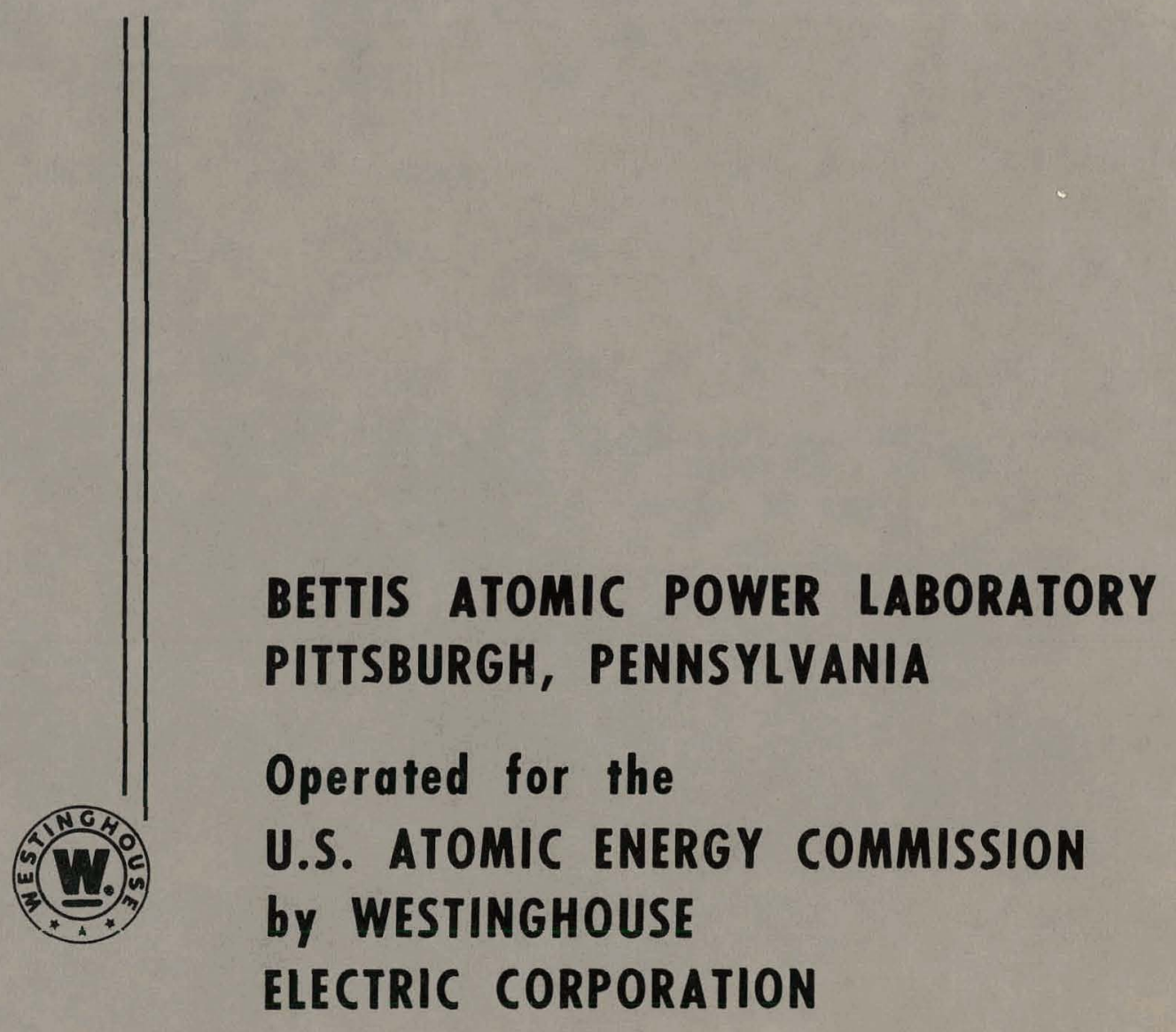




\section{DISCLAIMER}

This report was prepared as an account of work sponsored by an agency of the United States Government. Neither the United States Government nor any agency Thereof, nor any of their employees, makes any warranty, express or implied, or assumes any legal liability or responsibility for the accuracy, completeness, or usefulness of any information, apparatus, product, or process disclosed, or represents that its use would not infringe privately owned rights. Reference herein to any specific commercial product, process, or service by trade name, trademark, manufacturer, or otherwise does not necessarily constitute or imply its endorsement, recommendation, or favoring by the United States Government or any agency thereof. The views and opinions of authors expressed herein do not necessarily state or reflect those of the United States Government or any agency thereof. 


\section{DISCLAIMER}

Portions of this document may be illegible in electronic image products. Images are produced from the best available original document. 
EURIPUS-3 AND DAEDALUS-MMNTE CARLO DENSITY CODES FOR THE IBM-704

Harvey J. Amster

Heidi G。 Kuehn

Jerome Spanier

Contract $\cdot$ AT-1. $-1-$ GEN-14

February 1960

Price $\$ 2.25$

Available from the Office of Technical Services,

Department of Commerce,

Washington 25, D。C。

NOTE

This document is an "interim memorandum prepared primarily for internal reference and does not represent a final expression of the opinion of Westinghouse. When this memorandum is distributed externally, it is with the express understanding that Westinghouse makes no representation as to completeness, accuracy, or usability of information contained therein.

BETTIS ATOMIO TOWER LABORATORY

PITTSBURGH, PENNSYLVIANIA

OPERATED FOR THE U. S. ATOMIC ENERGY COMMISSION BY

WESTINGHOUSE ELECTRIC CORPORATION 
Director, Development Division, PNROO, AEC.

Manager, Pittsburgh Naval Reactors. Operations Office, AEC 10

Argonne National Laboratory, W. F. Miller

Brookhaven National Laboratory, J. Chernick

Brookhaven National Laboratory, M. Rose

Case Institute of Technology, R. S. Varga

David Taylor Model Basin, H. Polachek

Knolls Atomic Power Laboratory, R. Ehrlich

Los Alamos Scientific Laboratory, B. Carlson

New York University, R. Richtmyer

Oak Ridge National Laboratory, V. E. Anderson

Oak Ridge National Laboratory, A. Householder

University of California Radiation Laboratory, Livermore, S. Fernbach

This report was prepared as an account of Government sponsored work. Neither the United States, nor the Commission, nor any person acting on bchalf of these agencies:

A. Makes any warranty or representation, expressed or implied, with respect to the accuracy, completeness, or usefulness of the information contained in this report, or that the use of any information, apparatus, method, or process disclosed in this report may not infringe privattely: owned rights; or

B. Assumes any liabilities with respect to the use of, or for damages resulting from the use of any information, apparatus, method, or process disc1oned in thic report.

As used in the above, "person acting on behalf of these agencies" includes any employe or contractor of these agencies to the extent that such employe or contractor prepares, handles or distributes, or provides access to, any information pursuant to his cmployment or contract with the Commission. 


\section{TABLE OF CONTENTS}

\section{$\underline{\text { Page }}$}

ABSTRA:CT

iv

PART I: ANALYTIC AND PHYSICAL DESCRIPTION

PART II: STATISTICAL ANALYSIS

PART III: EXAMPLES TESTING FORMULATION AND ACCURACY

PART IV: A PROOF THAT DAEDALUS IS UNBIASED

PART V: CODE OPERATION 


\section{A.BSTRACT}

EURIPUS-3 calculates the onemdimensional spatial density of neutrons slowing-down past a given energy in an infinite homogeneous medium consisting of hydrogen and one other isotope with arbitrary mass and energy-dependent differential-elastic and absorption cross sections. DAEDALUS determines the corresponding spatial distribution of angular integrals of an arbitrary function times the vector flux density. Spatial moments of all density functions are furnished directly. Although scattering angles are calculated by Monte Carlo, the spatial distributions and, in DAEDALUS, the energy distribution are obtained partly from an analytic treatment which, besides saving time, enables the output to be in the form of actual density functions at specified planes and energies, rather than histograms covering finite intervals. At certain steps in the computation of both the spatial and energy distributions, part of the analytic treatment is replaced by Monte Carlo in order either to maximize efficiency and/or to avoid round-off error. The neutron source may be monoenergetic with either isotropic or monodirectional angular distributions, or else the source may be that from deuterons bombarding deuterons. The volume displaced by a cylindrical tube from an accelerator to the source can be accounted, for in the neutron first flight but not thereafter. 


\section{PART I: ANALYTIC AND PHYSICAL. DESCRIPTION}

Harvey J. Amster

\section{I。 INTRODUCTION}

The codes here described were produced for three reasons: to test neutron cross sections by comparing accurate calculations with foil-activation experiments (Ref.1), to test the approximations of transport codes (Ref。2) in an extreme situation, and to evaluate the benefits of combining Monte Carlo with analytic calculations.

The density functions calculated by these codes are somewhat unusual for Monte Carlo: Each history of EURIPUS yields a sample of the limit as $\Delta z$. goes to zero of $1 / \Delta z$ times the number of neutrons per second that slow down past a given energy at distances between $z$ and $z+\Delta z$ from a plane containing the source; each history of DAEDALUS calculates a sample of the limit, as $\Delta z$ and $\Delta E$ go to zero, of $1 /(\Delta z \Delta E)$ times the number of neutrons between $z$ and $z+\Delta z$ that have energies between $E$ and $E+\Delta E$ (the neutrons in $\Delta E$, however, have a unique direction for each history). Both codes directly provide the spatial moments of each density function. Unlike pure analog Monte Carlo, which could furnish an estimate of the density functions only for finite intervals $\Delta \mathrm{E}$ and $\Delta \mathrm{z}$ and only by using the results of many histories, the present codes yield an estimate of the density functions at all specified distances for each history. The possibility for such output is due to the analytic treatment, which will now be described。 


\section{DERIVATION OF SPATIAL DENSITY FUNCTIONS}

In a homogeneous medium, the directions and energies of a particular neutron alowing down can be calculated by Monte Carlo without reference to the location of each colligion. With these angles and energies as parameters; it is possible to calculate the probability that the neutron had any particular collision between parallel planes at distances $z$ and $z+d z$ from the point of origin. The following derivation is for an infinite medium, and is similar to a previous treatment for the diffusion of gamma rays" (Ref: 3) 。

The probability that a neutron departing at a distance $z^{p}$ from a plane containing the source, will be scattered at distances between $z$ and $z+d z$ from the source plane is

$$
\begin{aligned}
Q\left(z^{8}, z\right) d z & =\frac{\Sigma^{S}}{|\mu|} \exp \left(z^{8}-z\right) \frac{\Sigma^{T}}{\mu} d z \text { if the exponent is negative } \\
& =0
\end{aligned}
$$

where $\mu$ is the cosine of the angle between the direction of travel and the $z$ axis, and $\dot{\Sigma}^{3}$ and $\dot{\Sigma}^{T}$ are the macroscopic scattering and total cross sections of the medium. The zero contribution to $\mathrm{Eq}$. (1) states simply that there is no chance 
that a neutron heading in a given direction can have its next collision behind its point of origin. Note that Eq. (I) properly accounts for absorption.

Let $P_{i}(z) d z$ be the probability that a neutron, furnished by the source at $z=0$, will have an $i+l$ st scattering collision in the interval $d z$ 。 Evidently,

$$
P_{0}(z)=Q_{0}(0, z),
$$

where the subscript $i$ on $Q_{i}\left(z^{\prime}, z\right)$ will from now on mean that in Eq. (I) $\mu=\mu_{i}$ s $\Sigma^{S}=\Sigma_{i}^{S}$, and $\Sigma^{T}=\Sigma_{i}^{T}$, the values of these parameters that affect the neutron immediately after its collision i.

$$
\begin{aligned}
& P_{i}(z) \text { for } i>0 \text { can be obtained from } \\
& \qquad P_{i}(z)=\int_{-\infty}^{\infty} P_{i-1}\left(z_{i}\right) Q_{i}\left(z_{i}, z\right) d z_{i} .
\end{aligned}
$$

Equation (3) states that the scattering rate for the $i+1$ st collision is a sum of contributions from all possible positions of the previous collision $i$; the amount from the positions in $\mathrm{dz}_{i}$ is the local scattering rate times the probability that the next collision of a neutron departing from $z_{i}$ will be a scattering in $\mathrm{dz}$.

Aissume the solution to Eq. (3) is of the form

$$
P_{i}(z)=\left|\sum_{k=0}^{i} D_{k}^{i} e^{-\beta_{k} z}\right|
$$

where $\alpha_{\mathrm{k}}=\Sigma_{\mathrm{k}}^{\mathrm{S}} / \mu_{\mathrm{k}}$ and $\beta_{\mathrm{k}}=\cdot \Sigma_{\mathrm{k}}^{\mathrm{T}} / \mu_{\mathrm{k}}$, and where an exponential to a positive power is understood to be identically zero. A substitution of Eq. (4) into Eqs. (2) and (3) show that regardless of the signs of $z$ and the $\beta_{k}{ }^{\prime} s$, Eq. (4) will be true if 


$$
\begin{aligned}
& D_{0}^{0}=\alpha_{0} \\
& D_{k}^{i}=\frac{\alpha_{i} D_{k}^{i-1}}{\beta_{i}-\beta_{k}} \text { for } k=0 \text { thru } i-1 \\
& \vdots D_{i}^{i}=-\sum_{k=0}^{i-1} D_{k}^{i} .
\end{aligned}
$$

A practical difficulty in using Eq. (4) is that the magnitudes of the individual terms may become very much larger than their sum, since individual terms may be positive or negative. Very severe round-off errors have been found to occur on a digital computer, usually becoming worse as the number of collisions increases.

To avoid this difficulty, the present codes test for the possibility of round-off error after the calculation of each $D_{k^{0}}^{i}$ If such a possibility exists after the $t$ th collision, the codes calculate by straight analog Monte Carlo $z_{t^{\prime}}$ the $z$ coordinate of the $(r+1)$ st collision minus that of the $(t+1)$ st (Sect. $V$ will show that. DAEDALUS makes a source-tube size correction by adding a length $z_{T}$ to this definition of $\left.z_{t r}\right)$. $w_{t r}$, the probability that a neutron having completed its $(t+1)$ st collision will remain unabsorbed immediately after its $(r+1)$ st collision, is also calculated. Then use is made of the relationship that an . average over many histories of the quantity

$$
P_{r}(z)=w_{t r} P_{t}\left(z-z_{t r}\right)
$$

for any $t$ between 0 and $r$, will equal that from the definition of Eq. (4).

EURIPUS calculates $q(z)$, the rate that neutrons will slow down past the energy $E_{R}$ at spatial points in $d z$. For each history, the estimate of $q(z)$ is $P_{r}(z)$ where $r$ is defined from

$$
E_{r}>E_{R} \geq E_{r+l}
$$

and where $E_{i}$ is the energy of a neutron immediately after its i'th collision. 
Before describing DAEDALUS, we now relate the above discussion with some previous work. A more formal calculation (Ref。4) than the intuitive derivation above has produced the explicit solution to Eq. (5),

$$
D_{k}^{1}=\prod_{j \neq k}^{1}\left(\beta_{j}-\beta_{k}\right)^{-1}
$$

EURIPUS-3, however, uses Eq. (5) becanse it requires fewer numerical steps. EURIPUS-2, a test code previous to EURIPUS-3, calculated $P_{\mathbf{Y}}(\mathrm{z})$ by treating the displacement between collision points entirely analytically, and thus never utilized $\mathrm{Eq}$ (6). The discovery of round off error was made through the use of EURIPUS-2, which does not check such dangers and, therefore, now has very limited usefulness. The calculational method was derlved from an intultive argument simflar to the above, but considered last colliaions firsto $\mathcal{B}_{k}$ was called $c_{k}^{0}$ and was obtained from the backward recursion formulas,

$$
\begin{aligned}
& c_{r}^{r}=a_{r} \\
& c_{k}^{i}=\frac{a_{i}}{\beta_{1}-\beta_{k}} c_{k}^{i+1} \text { for } k=0 \text { thru } 1-1 \\
& c_{i}^{i}=-\sum_{k=1+1}^{r} c_{k}^{i}
\end{aligned}
$$

It was decided to treat the first collisions analytically, rather than the last, in EURIPUS-3 beceuse most cross sections are smaller at high energies and are more important in determining the finel spatial distributions, $\mathrm{P}_{\mathrm{r}}(\mathrm{z})$ 。 Forward recursion formulas were used because a dectsion could be made at each recursion whether or not another collision could be handled analytically. In either case, the numerical results of Eq. (5) could be used。 If the backward recursions of $\mathrm{Eq}$. (9) had been used, starting at some intermediate collision and 
working back to the source, and if round off error were discovered before the source were reached, all the recursions would have to be repeated, starting at some new intermediate collision。

EURIPUS-1, a test code previous to EURIRUS-2, treated only the last collision analytically-by a single exponential being extended from the position of the $r$ th collision. This was the simplest code giving a density function and has been used to check the two later versions. More histories per unit time are calculated by EURIPUS-1 than by EURIPUS -2 becaise of the recursion formalas and the several exponentials in Eq. (4). Evidentiy, it does not pay, in terms of final accuracy, to spend too much time in calculating an accurate spatial distribution analytically for a given set of path directions, since the path directions themselves are furnished by Monțe Carlo and should be well sampled. Thus, it was made possible in EURIPUS-3 to limit the number of analytic calculations by an input number as well as by the self-testing round off error critieria.

EURIPUS-2 can have a Ascontinulty in the slowing down denisity output only at the origin (for a finite number of histories), while EURIPUS-1 may have them anywhere. EURIPUS-3. máy have discontinuities anywhere, but they will be smaller than the previous versions and will be unlikely far from the origin. No reports on EURIPUS- 1 and -2 have been issued because EURIPUS- 3 is belleved to incorporate all of the best properties of both.

\section{HOW DAEDALUS CALCULATES FLUX AND CURRENT DENSITIES}

The problem considered is how to calculate the flux and current of neutrons at locations in $d z$ and with energies between the specified value $E_{R}$ and $E_{R}-d E_{R^{\circ}}$ Although not essential to the formilation, a situation almost always true in practical problems was assumed: only isotropic scattering in the center of mass system can occur for collisions which might slow a neution to an energy below $\mathbf{E}_{\mathbf{R}^{\circ}}$ 
DAEDALUS starts out the same way as EURIPUS, except that, since one more collision will be involved, we define $r$ as being one larger than does $\mathrm{Eq}$ (7):

$$
E_{r-1}>E_{R} \geqslant E_{r}
$$

When the neutron had energy $\mathrm{I}_{\mathrm{r}-1}$, the probabillty density for being acattered Into a lower energy interval was constant for exit energies above $\alpha E_{r-1}\left(\alpha<I^{\prime}\right.$ and depends. only on the mass of the scatterer), and zero, below (Ref。 5)。 Thus the ratio of the number of neutrons that scatter into $\mathrm{dE}_{\mathrm{R}}$ in one collision the number that scatter below $E_{R}$ is

$$
\frac{d E_{R}}{E_{R}-a E_{r-1}}
$$

Once Monte Carlo has established the fact, through Eq. (10), that a collision $r$ will change the energy from $E_{r-1}$ to some value below $E_{R}$, then the above ratio. can be used to give a sample of the net flow of neutrons from $E_{r-1}$ into $\mathrm{dE}_{\mathrm{R}^{\circ}}$ This step is possible because, in the absence of "upscattering", all neutrons that are scattered to energies below the interval $\mathrm{de}_{\mathrm{R}}$ will never be scattered into $d E_{R^{2}}$ and with $d E_{R}$ infinitesimal, all neutrons scattered into $d E_{R}$ once will never be scattered in again。

Now $P_{r-1}(z) d z_{g}$ with $r$ defined by Eq. (10), gives the number of neutrons In $\mathrm{dz}$ that do scatter below $\mathrm{E}_{R}$ in a particular history。 Thus, the rate of neutrons flowing from $E_{r-1}$ to $d E_{R}$ at positions in $\mathrm{dz}$ is

$$
\frac{P_{X-1}(z) d z d E_{H}}{E_{R}-a E_{I-1}} \text {. }
$$

It may seem strange that the slowing in source of neutrons can be evaluated at an arbitrary energy $\mathrm{F}_{\mathrm{R}}$ when the Monte Carlo describes the history as never actually having had this energy. The explanation lies in the fact that the energy 
$E_{r}$, below $E_{R}$, is never used for any other reason than to identify the collision number $r$, according to Eq. (10), where the Monte Carlo calculation ends. The scattering angle $\mu_{R-1}^{S}$ and the energy $E_{R}$ of the "R th collision", to be substi-: tuded for the $\mathbf{r}$.th as will now be described, are calculated analytically and involve no random numbers.

To be conventional, we now speak of neutrons in the lethargy interval $d u_{R}=d E_{R} / E_{R}$ at energy $E_{R}$, so that the slowing in source becomes

$$
\frac{P_{r-1}(z) d z d u_{R}}{1-\alpha \frac{E_{j-1}}{E_{R}}}
$$

By the conservation of energy and momentum, it is possible to calculate $\mu_{\mathrm{R}-1}^{\mathrm{S}}$, the scattering angle undergone by a neutron in going from energy $E_{r-1}$ to $\mathrm{E}_{\mathrm{R}}$. From $\mu_{\mathrm{R}-1}^{\mathrm{S}}, \mu_{\mathrm{r}-1}$, and the azimuthal angle of scattering, one can calculate $\mu_{R}$, the cosine of the angle between the $z$ axis and the direction of those neutrons which were slowed down into the interval $d_{R^{\circ}}$

The flux of neutrons in any interval $\mathrm{dz}$ is an integral of the contributions from the slowing in source at all positions. The contribution from each position is the source of neutrons at that spot times $1 / \Sigma^{S}\left(E_{R}\right)$ the chance that a neutron from there will be scattered in $\mathrm{dz}$ :

$$
p\left(u_{R^{\prime}} z\right)=\frac{1}{1-\alpha E_{r-1} / E_{R}} \int_{-\infty}^{\infty} P_{r-1}\left(z_{R}\right)\left[\frac{Q_{R}\left(z_{R}, z\right)}{\Sigma_{R}^{S}}\right] d z_{R} ;
$$

where the subscript $R$ on $Q$ means that in Eq. (I), $\mu=\mu_{R}, \Sigma^{S}=\Sigma_{R}^{S}, \Sigma^{T}=\Sigma_{R}^{T}$, cross sections evaluated at the energy $E_{R^{*}}$ A comparison with Eqs. (1) to (5) shows that 


$$
\varphi\left(u_{R}, z\right)=\frac{P_{R}(z)}{\Sigma_{R}^{S}\left(1-\alpha E_{r-1} / E_{R}\right)}
$$

where $P_{R}(\dot{z})$ is exactly like $P_{r}(z)$ except that $\alpha_{r}$ and $\beta_{r}$ should be replaced by $\alpha_{R}=\Sigma_{R}^{S} / \mu_{R}$ and $\beta_{R}=\Sigma_{R}^{T} / \dot{\mu}_{R}$.

Besides calculating the scalar flux density $\varphi$ for each history, DAEDALUS also evaluates the activation of a thin "black" foil,

and

$$
\left|\mu_{\mathrm{R}}\right| \varphi
$$

$$
F\left(\mu_{R}\right) \varphi,
$$

where $F\left(\mu_{R}\right)$ is an arbitrary tabular function used $e_{0} g$ o to calculate the activation of a "gray" foil or any Legendre component of the vector flux.

Independently of the results of the "R th" collision, more collisions after the $r$ th could be continued, and evaluations made at lower energies. "Thus it is possible, in principle, to obtain a sample of the vector flux at all energies and positions from a single Monte Carlo history. However, DAEDALUS makes an evaluation only at a single energy and calculates only. the functions of the vector flux given by Eqso(15) to (17)。

Since $E_{\text {rol }}$ might take on any value between $E_{R}$ and $E_{R} / \alpha_{\text {, }}$ it is possible for $\varphi$ in Eq. (15) to become infinite. That is, if a neutron happens to have an. energy. fust slightly below $E_{R} / \alpha$, its chance of slowing down below $E_{R}$ may become so small that the ratio in Eq. (13) divided by $\mathrm{du}_{\mathrm{R}} \mathrm{dz}$ may "blow up." Such difficulties would seem rare in practice because not only does $E_{r-1}$ have to be just below $E_{R} / \alpha$ for a machine overflow to be possible, but for the overflow actually to occur, the Monte Carlo must also place $E_{r}$ in the small interval between $\alpha E_{r} \rightarrow$ and $E_{R^{g}}$ so that $r$ will be identified according to Eq. (10)。 Although the possibility of a machine overflow seems remote, it would be beneficial to avoid Eq. (15) when the difference between the two terms in the denominator is in 
danger of becoming small because the percentage round-off error would be large for large contributions. Furthermore, reducing the possibility of the infinite contribution would seem to tighten the distribution of flux contributions in: general and "improve statistics".

In DAEDALUS, if a collision is recognized as "risky" in view of its energy and target species (when hydrogen is the scatterer, the above difficulties are not present because the $\alpha$ of hydrogen is zero), the history is split to avoid any singularity in calculation. That is, if a collision $j+I$ is with the nonhydrogenous moderator and the incident energy $E_{j}$ is such that

$$
E_{R} / \alpha>E_{j} \geq f E_{R} / \alpha
$$

where the fraction $f$ is an input number; it is recognized that the chance that the exit energy $E_{j+l}$ will be in $d_{R}$ is

$$
\frac{d E_{R}}{(1-\alpha) E_{j}} \text {. }
$$

The flux from these particular neutrons can be calculated analytically in a fashion similar to the one leading to $\mathrm{Eq}$. (15), and there results

$$
\varphi_{1}\left(u_{R}, z\right)=\frac{P_{R}(z)}{\Sigma_{R}^{S}(1-\alpha) E_{j} / E_{R}}
$$

Besides those neutrons scattering from $E_{j}$ directly into $d E_{R}$, contribu-. tions to the flux in $\mathrm{dE}_{\mathrm{R}}$ are possible for the fraction of neutrons whose values of $E_{j+1}$ are above $E_{R}$ i this fraction is

$$
y=\frac{E_{j}-E_{R}}{E_{j}(1-a)} .
$$

Monte Carlo can be used to get this second contribution to the flux by continuing the standard Monte Carlo procedure ending with Eq. (15), except that (1) the 
weighting factor $\mathrm{y}$ should multiply the answer, and (2) the energy $\mathrm{E}_{j+1}$ must be picked by Monte Carlo from a distribution between $E_{j}$ and $E_{R}$ (since we have already accounted for those below $\left.E_{R}\right)$, $y$ and the coefficient of $P_{R}(z)$ in Eq。(19) cannot become infinite!

Although a procedure similar to the above could be extended to eliminate the danger that collisions subsequent to the risky collision could also be risky, the actual danger involved is considered negligible. The above procedure has not only drastically reduced the chance for abnormally large contributions to the flux with serious round-off error, but by treating more of the collision analytically, it would be expected to reduce the statistical spread for a given number of histories. As with the spatial distribution, however, the extra analytic treatment has also resulted in requiring more computing time per history, but since risky collisions themselves are usually rare, the issue of efficiency here is a much less serious one.

\section{SPATIAL MOMENTS}

Having obtained an analytic expression for spatial densities, one can now calculate their moments directly. Equations (4) and (6) show that the moments of the various density functions are linear combinations of the moments of the functions

$$
\begin{aligned}
& f_{k}(z)=\exp -\beta_{k}\left(z-z_{t r}\right) \text { if the exponent is negative } \\
&=0 \\
& \text { if the exponent is positive. }
\end{aligned}
$$

Both full range moments $\nu_{k}^{K}$ and half range moments $\tau_{k}^{K}$ are desired:

$$
v_{k}^{K}=\int_{-\infty}^{\infty} \cdot f_{k}(z) z^{K} d z ; \quad \tau_{k}^{K}=\int_{0}^{\infty} f_{k}(z) z^{K} d z
$$


These integrals can be evaluated analytically to provide the following expressions for the moments:

$$
\begin{aligned}
& b_{k}^{K} \equiv(K+I) / \beta_{k} \text { is calculated from } b_{k}^{O}=1 / \beta_{k} \text { and } b_{k}^{K}=b_{k}^{0}+b_{k}^{K-I} \\
& a_{k}^{K} \equiv\left(z_{t r}\right)^{K+1} / \beta_{k} \text { is calculated from } a_{k}^{O}=z_{\operatorname{tr}} b_{k}^{0} \text { and } a_{k}^{K}=z_{t r} a_{k}^{K-1} \\
& \mathrm{U}_{\mathrm{k}}^{\mathrm{O}}=\mathrm{b}_{\mathrm{k}}^{\mathrm{O}} \exp \beta_{\mathrm{k}} \mathrm{z}_{\mathrm{tr}} \text { and } \mathrm{U}_{\mathrm{k}}^{\mathrm{K}}=\mathrm{b}_{\mathrm{k}}^{\mathrm{K}-1} \mathrm{U}_{\mathrm{k}}^{\mathrm{I}-1} \\
& v_{k}^{O}=v_{k}^{O} . \quad \text { and } v_{k}^{K}=a_{k}^{K-1}+h_{k}^{K-1} v_{k}^{K-1} \\
& \text { For } z_{t_{r}} \leq 0 ; \quad \tau_{k}^{K}=0 \text { if } \beta_{k} \leq 0 \\
& =U_{k}^{K} \text { if } \beta_{k} \geq 0 \\
& \text { For } z_{t r} \geq 0, \quad \tau_{k}^{K}=v_{k}^{K}-U_{k}^{K} \text { if } \beta_{k} \leq 0 \\
& =v_{k}^{K} \quad \text { if } \beta_{k:} \geq 0
\end{aligned}
$$

\section{TUBE STLE CORRECTION}

The discussion of sects. II through IV was for a completeily homogentzol infinite medium with the source restricted to a plane. Actually, for a D-D source of neutrons, an evacuated tube must be inserted to allow the deuterons from the accelerator to reach those of the target producing the source neutrons. In DAEDALUS, the source of neutrons is idealized as a point at the centroid of the actual source volume. The resulting geometry is displayed in Fig. 1, where a semi-infinite empty cylindrical volume of radius $\rho$ contains the 
source point on its axis a distance $\ell$ from the end of the tube. In such a situation, the probability for the first scattering collision to occur between planes at $z$ and $z+d z$ distances from the source is zero for $z<z_{T}$, the $z$-projection of the length of free flight within the tube. Past the tube, the function for the probability density of the first collision is the function that would occur if the tube were absent and the source were displaced to the right by $z_{T^{\circ}}$

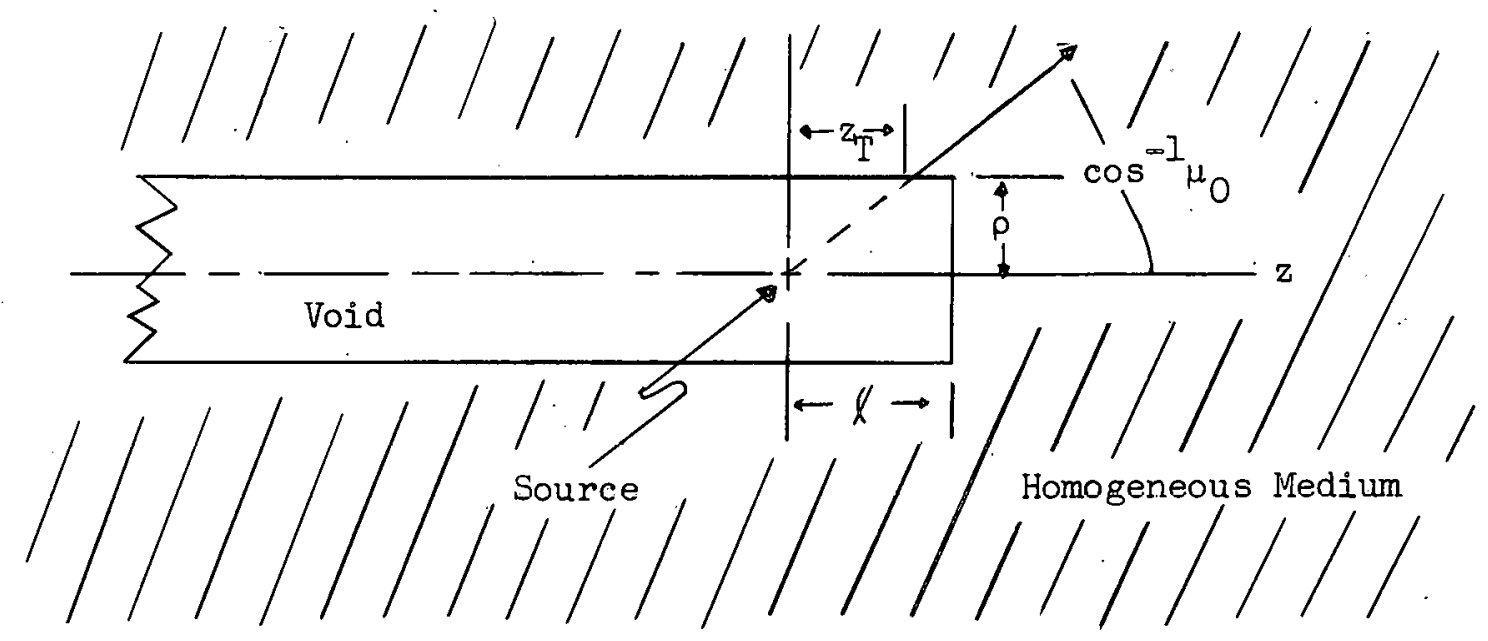

Fig. 1. Geometry of Source in Tube

Thus, the tube correction for the first flight is simple; furthermore it is important, since every source neutron makes a free fight within the tube. On the other hand, a tube correction for subsequent collisions would be very difficult to handle because a threemdimensional geometry is involved. Fortunately, such a correction should be relatively small since, once a neutron has left the tube into the surrounding volume, there is only a small chance that it will ever reenter the tube. The flux in the vicinity of the tube will of course be affected 
by the presence of the void, but we are concerned with all the neutrons between the infinite planes at $\mathrm{z}$ and $\mathrm{z}+\mathrm{dz}$ 。

Therefore, DAEDALUS makes only a first flight tube correction by assuming the flux density at any energy has the spatial distribution that would occur if the tube were not present, displaced by $z_{T}$. That is, the $z_{t r}$ in $E_{q} \cdot(6)$ should be the difference in the $t+1$ st and $r+1$ st $z$ coordinates plus $z_{T}$ s where

$$
\begin{aligned}
z_{\mathrm{iT}} & =\Omega & & \text { if } \ell=\rho=0 \\
& =\ell & & \text { if } \mu_{0} \geq \chi\left(\ell^{2}+\rho^{2}\right)^{-1 / 2} \\
& =\rho \mu_{0}\left(1-\mu_{0}^{2}\right)^{-1 / 2} & & \text { if } \mu_{0}<\chi\left(\ell^{2}+\rho\right)^{-1 / 2}
\end{aligned}
$$

VI. SOURCE PREPARATION ROUTINE

The neutron source may be

(1) Monoenergetic and monodirectional: $E_{O}$ and $\mu_{0}$ inputted。

(2) Monocncrgetic and iootropios $E_{0}$ inputted, and $\mu_{0}=1-2 \xi$ ( $\xi$ will always represent a random number).

(3) That from the deuteron-deuteron reaction: the incident deuteron energy $E_{D}$, the reaction energy $Q$, and the Legendre coefficients $S_{L}$ for the angular distribution of the exit neutron in the center-of-mass system are inputted. A: table is then calculated that gives the exit neutron energy as a function of random number. After this source energy is chosen, the corresponding angle $\mu_{0}$. is calculated by conservation of momentum and energy. This procedure results in shorter equations than does the alternate possibility of choosing the direction of the source neutron from Monte Carlo and calculating the energy from the angle. If the relative probability for the source neutron to be emitted into the solid angle $2 \pi \mathrm{d}^{c}$ in the C.M. system is given by 


$$
2 \pi S\left(\mu^{c}\right) d \mu^{c}=\sum_{L=0}^{N} \frac{2 d_{L}+1}{2} S_{L_{L}} P_{L}\left(\mu^{c}\right) d \mu^{c},
$$

where $N$ is an input number less than $16, \mu^{c}(\xi)$ could be calculated by Monte Carlo fron

$$
\xi=\frac{2 \pi \int_{-1}^{\mu^{c}} S(\mu) d \mu}{2 \pi \int_{-I}^{1} S(\mu) d \mu}=\frac{1}{2 S_{0}} \sum_{L=0}^{N} s_{L}\left[P_{L+1}\left(\mu^{c}\right)-P_{L-1}\left(\mu^{c}\right)\right],
$$

where the Legendre polynomials are obtained from the recursion formulas,

$$
\begin{aligned}
& P_{-I}(\mu)=-1, P_{0}(\mu)=+1 \\
& P_{L+1}(\mu)=\frac{2 L+1}{L+1} \mu P_{L}(\mu)-\frac{I}{L+I} P_{L-1}(\mu) 。
\end{aligned}
$$

However, we desire the Monte Carlo to fumish the energy of the source neutron,

$$
E_{0}\left(\mu^{c}\right)=\frac{1}{2} E_{D}+\frac{3}{4} Q+\frac{\sqrt{3}}{4} \cdot \sqrt{E_{D}\left(E_{D}+2 Q\right)} \mu^{c} \text {. }
$$

A table of $E_{0}\left(\mu^{c}\right)$ and $\xi\left(\mu^{c}\right)$ are calculated from Eqso (35) and (37) for various values of $\mu^{c}$ 。 Then these tables are combined to yleld $E_{0}(\xi)$ at specified points, between which linear Interpolations are performed. The integer m, less than 50 , 1s inputted, and the tables are calculated fron the +1 evenly spaced values of $\mu^{\mathrm{c}}$,

$$
H_{p}^{c}=-1+\frac{2 p}{m} \quad \therefore y=0 \text { to } m
$$

Finally, the source preparation routine furnishes the constants

$$
a=\sqrt{2 / E_{D}} \quad \text { and } \quad b=-\frac{1}{2}(1 / a+3 Q a / 2) \text {, }
$$


from which the source direction (in lab system) can be calculated in the nain portion of the codesis

$$
H_{0}=a E_{0}^{1 / 2}+b E_{0}^{-1 / 2}
$$

\section{CROSS SECTION LIBRARY ROUTINE}

The cross sections denoted by the subscript $n$ are constant in the energy Interval between the input numbers $\mathrm{E}_{\mathrm{n}}$ and $\mathrm{E}_{\mathrm{n}-1^{\circ}} \mathrm{n}$ Lchreases with lethargy, so that $E_{n}<E_{n_{-1}} 1^{\circ}$. The values taken on by $n$ include all the integers 0 thru $k_{\text {g another }}$ input number less than 401 .

For each $n$, there is inputted: the group energy $E_{n} 8$ the total and scattering cross sections of hydrogen, $\sigma_{n}^{\mathrm{TH}}$ and $\sigma_{n}^{\mathrm{SH}} ;$ the total cross section and the Legendre components of the scattering cross section of "moderator A" (the non-hydrogenous 18otope), $\sigma_{n}^{T A}$ and $B_{I n}{ }^{i} s$ the value of the highest $I$ used, $N_{n}$, an Integer less than 21 , the quantity $\delta_{n}$ whose two possible values, 0 and 1 , specify respectively whether the differential cross sections of moderator $A$ are in the C.M. or lab systems and $m_{n^{2}}$ an integer less than 40 specifying the number of miesh points used in describing the differential cross section to the main body. In addition there is inputted $A_{9}$, the mass of moderator $A_{9}$ and $N^{A}$ and $N^{H}$, the number densities of moderator $A$ and hydrogen.

The Iibrary routine furnishes the main body of EURIPUS and DAEDALUS with $A_{9}$. for each $n_{8}$ the macroscopic cross sections,

$$
\begin{aligned}
\Sigma_{n}^{S A} & =N^{A} B_{O n} \\
\Sigma_{n}^{S} & =\Sigma_{n}^{S A}+N^{H} \sigma_{n}^{S H} \\
\Sigma_{n}^{T} & =N^{A} \sigma_{n}^{T A}+N^{H} \sigma_{n}^{T H},
\end{aligned}
$$


and a table of $\mu_{\mathrm{n}}^{\mathrm{S}}(\xi)$, the lab system cosine of the angle of scattering by moderator $A$ as a function of the random numbers to be chosen later in the main body.

The table $\mu_{n}^{S}(\xi)$ is calculated very analogously to the manner in which $\mu^{c}(\xi)$ is obtained in Eq. (35). The differential scattering cross section per unit solid angle for scattering into angle $\cos ^{-1} \eta$ is expressed as

$$
\sigma_{n}(\eta)=\sum_{I=0}^{N} \frac{2 I+1}{4 \pi} B_{L n} P_{L}(\eta)
$$

For each random number $\xi$, there corresponds a value $\eta$ given by

$$
\xi=\frac{1}{2 B_{0 n}} \sum_{I=0}^{\mathbb{N}_{n}} B_{I_{\text {on }}}\left[P_{I+I}(\eta)-P_{I_{-\infty} I}(\eta)\right]
$$

where the Legendre polynomials are calculated from $\mathrm{Eq}_{0}$ : (36)。 For a given $\xi$, what is actually desired is the lab systein scattering angle $\mu_{n^{\circ}}$. Thus,

$$
\begin{aligned}
\eta & =\mu_{n}^{s} \sqrt{1-\left[1-\left(\mu_{n}^{s}\right)^{2}\right] / A^{2}}-\left[1-\left(\mu_{n}^{s}\right)^{2}\right] / A \text { if } \delta_{n}=0 \\
& =\mu_{n}^{s} \quad \therefore \text { if } \delta_{n}=i
\end{aligned}
$$

By using Bqs. (43) and $(44), \xi\left(\mu_{n}^{S}\right)$ is calculated for the $m_{n}+1$ values of $\mu_{n}^{S}$,

$$
\mu_{n, p}^{s}=-1+\frac{2 p}{m_{n}} \quad: \quad p=0 \text { to } m_{n}
$$

Then the main portion of the code interpirets the table as $\mu_{n}^{S}(\xi)$ and linearly Interpolater botween the tabulated values.

VIII. THE MAIN PORTION OF THE CODES

In addition to the information obtained from the source preparation and cross section library routines, the matn portions of both EURIPUS and DAEDALUS 
require as input the following quantities:

1. $E_{R}$, the energy at which the output quantities are to be evaluated.

2. $M$, an integer $\leq 100$, equal to the number of spatial positions at which the z-dependent quantities are to be evaluated.

3. The $M$ values of $z$, the distances (with algebraic sign) from the : source plane.

4. $P$, an integer $\leq 10$, equal to the highest order (value of $K$ in Eq. (22)) moments to be evaluated.

5. $\mathrm{N}$, an integer $\leq 100$, denoting that $N+1$ collisions $(i=0$ thru N) per history should have their spatial distributions calculated analytically if such a procedure is not interrupted either by failing the round-off error test or by the energy of the neutron falling below $E_{R^{*}}$

6. $\varepsilon$, a positive two digit integer, giving the number of digits to the right of the decimal point which will not be affected by round-off error in the evaluation of spatially dependent output quantities.

DAEDALUS requires the additional input quantities:

7. $\rho$ and $l$, the distances from the point source to the sides and end of its containing cylinder.

8. The splitting parameter $f$, denoting that neutrons colliding with moderator A (the non-hydrogenous isotope) with energies between $F_{\mathrm{R}} / \alpha$ and $f E_{\mathrm{K}} / \alpha$ will have their histories split.

9. An integer $M_{T}$ and the $M_{T}$ values of the function $F\left(\mu_{R}\right)$ at $\mu_{R}=-1$ to +1 in intervals of $2 /\left(M_{T}-1\right) .50 \geq M_{T} \geq 2$.

From A, furnished by the cross section library routine, the main code calculates:

$$
\alpha^{1 / 2}=(A-I) /(A+I) ; \beta=2(A+I)^{-2} ; \gamma=A^{2}-1 \text {. }
$$


The next stop starts the cal alation of an individsal history initiated with $E_{0}$ and $H_{0}$ as described in Soct; VIo Then DAEDALUS (but not EJRIPUS) calcuIates $\mathrm{z}_{\mathrm{T}}$ from Eqs. (31) to (33)。

At this point the code goes through a calculation which deteraines (1) at which collision, if any, in DAEDALUS the history should be split; (2) quanthities needed for the split historyg and (3) all the $\alpha_{1}{ }^{8}$ and $\beta_{1}{ }^{8}$ s that would be used in the andytic expressions if no round-off errors occur. That is, Monte Carlo calculates the energies and angles of a sequence of collistons and stops only ofter the collision number 1 has reached $p$, (the smaller) one of two integers: (1) $N$ inputted or (2) $r$, the last collision of the history, defined by

$$
E_{r}>E_{R} \geqslant E_{r+1} \text { for EURIPUS and } E_{r-1}>E_{R} \geqslant E_{r} \text { for DAEDALUS 。 }
$$

The celculation of the parameters of collision $1+1$ in terms of those of the previous collialon 1 and the random numbers $\xi$ will now be described. Any $\Sigma_{1}$ or $\mu_{i}^{S}(\xi)$ is taken, respectively, equal to the $\Sigma_{n}$ of Eq。 (4I) or $\mu_{n}^{S}(\xi)$ described after Eq. (45), where $n$ is defined from

$$
E_{n} \leqslant E_{1}<E_{n-1}
$$

A. If a $\xi>\Sigma_{1}^{S A} / \Sigma_{1}^{S}$, the $1+1$ at collision is with hydrogen, and 1. $\mathrm{E}_{1+1}=\xi \mathrm{E}_{1}$ from another $\xi$ 。

2. If $\mathrm{E}_{i+1} \leqslant \mathrm{E}_{\mathrm{R}^{\prime}}$ then $\mathrm{p}=r=1$ in EURIPUS, $\dot{p}=r=1+1$ in DAEDALUS, and In either code the history is ended.

3. If $\mathrm{sib}_{i+1}>\mathrm{R}_{\mathrm{R}}$

a If $1=N$, then $1=p$, and the calculation of the parameters for the analytic spatial description is completed.

b. Calculate $\mu_{1}^{s}=\sqrt{E_{1+1} / E_{1}}=\sqrt{\xi}$ of $1_{0}$ 
$B_{0}$ If $\xi$ of A. is such that $\xi \leqslant \Sigma_{i}^{S A} / \Sigma_{1^{9}}^{S}$ the $1+1$ st collision is with moderetor A. Then, DAEDALUS performs the following steps in order, while EURIPUS goes directly to: 2.d。

1.' If a previous collision was risky, $1_{0} e_{0}$ if $1>j$, the possibility of mother risky collision is ignored, and
a. $E_{1+1}=[\xi(1-a)+a] E_{1}$ 。
bo Steps A.2。 and A.3.a。
c. $\mu_{1}^{S}=\frac{A+1}{2}\left[\left(E_{i+1} / E_{i}\right)^{1 / 2}-\alpha^{1 / 2}\left(E_{1+1} / E_{1}\right)^{-1 / 2}\right]$.

2. If no previous collision was $\mathrm{risky}, 1_{0} \theta_{0}$ if $\mathrm{j}$ has.not been identified,

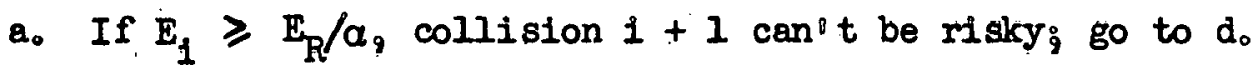

b. If $\mathrm{E}_{i}<\mathrm{E}_{\mathrm{R}} / \alpha$ and $\mathrm{E}_{1}<\mathrm{fE}_{\mathrm{R}} / \alpha$ also, collision $i+1$ is not risky; go to $d_{0}$

c. If $E_{R} / a>E_{1}>E_{R} / a$, collision $1+1$ is risky, $1=1$, the scattering is assumed isotropic in the CoM. system for all subsequent calculations, and there is calcalated

(1) the seattering angle for neutrong being scattered directly into $\mathrm{dE}_{\mathrm{R}^{9}} \mu_{\mathrm{R}-1}^{\mathrm{S}-\mathrm{i}}=\frac{A+1}{2}\left[\left(\mathrm{E}_{\mathrm{R}} / \mathrm{E}_{\mathrm{j}}\right)^{1 / 2}-\alpha^{1 / 2}\left(\mathrm{E}_{\mathrm{R}} / \mathrm{E}_{\mathrm{j}}\right)^{-1 / 2}\right]$

(2) the coefficient need for these neutrons in $\mathrm{Eq}$. (19), $x=E_{R} \div E_{1}(1-\alpha) \Sigma_{R}^{S}$

(3) the fraction of neutrons that scatter from $E_{f}$ to energies above $E_{R, 9} y=\left(E_{j}-E_{R}\right) \div E_{j}(1-\alpha)$

(4) the energy of the noxt collision (restricted to be above $E_{R}$ ), $\mathrm{E}_{j+1}=\mathrm{E}_{\mathrm{R}}+\xi\left(\mathrm{E}_{\mathrm{j}}-\mathrm{E}_{\mathrm{R}}\right)$

(5) the scattering angle corresponding to $\mathrm{E}_{\mathrm{j}+1^{2}}$

$\mu_{j}^{S}=\frac{A+1}{2}\left[\left(E_{j+1} / E_{1}\right)^{1 / 2}-a^{1 / 2}\left(E_{j+1} / E_{j}\right)^{-1 / 2}\right]$

d. If collision 1 and all previous collisions are not risky, calculate 
(1) $\mu_{i}^{S}=\mu_{i}^{S}(\xi)$ from the cross section library

(2) $E_{i+1}=E_{i}\left[\beta \mu_{i}^{S}\left(\mu_{i}^{S}+\sqrt{\left(\mu_{i}^{S}\right)^{2}+\gamma}\right)+\alpha^{I / 2}\right]$

(3) Steps A.2. and A.3.a.

C. Calculate the cosine of the scattering azimuthal angle by picking two random numbers, $\xi_{1}$ and $\xi_{2}$ 。

1. If $\xi_{1}^{2}+\xi_{2}^{2}>1$, pick two more $\xi^{\prime}$ s and repeat until $\xi_{1}^{2}+\xi_{2}^{2} \leq 1$ 2. $\cos \varphi=\left(\xi_{1}^{2}-\xi_{2}^{2}\right) /\left(\xi_{1}^{2}+\xi_{2}^{2}\right)$

D. Calculate $\mu_{i+1}=\mu_{i} \mu_{i}^{S}+\sqrt{\left[1-\left(\mu_{i}\right)^{2}\right]\left[1-\left(\mu_{i}^{S}\right)^{2}\right]} \cos \varphi$

E. Calculate $\alpha_{i}=\Sigma_{i}^{S} / \mu_{i}$ and $\beta_{i}=\Sigma_{i}^{T} / \mu_{i}$ for $i=0$ thru p and store.

Having obtained the needed values of $\alpha_{i}$ and $\beta_{i}$, EURIPUS calculates $D_{k}^{i}$ according to Eqs. (5). The $D_{k}^{i}$ are calculated for all the $k^{\prime} s$ ( 0 thru $i$ ) for a given $i$ before being calculated for an increment in $i$. If any $D_{k}^{i}$ would underflow the machine, its value is set equal to zero-ointroducing a possible but unlikely source of error when many recursions are performed.

In attempting to fulfili the requirement that $\varepsilon$ decimal places of the slowing down density $q(z)=P_{r}(z)$ be free of round-off error, EURIPUS has a provision that guarantees that $\varepsilon$ decimal places of each term in Eq. (4), with i set equal to $t$, will be correct (there is some chance that $q(z)$ will not have precisely the desired accuracy if the signs and magnitudes of the round-off errors in each term are such that they combine unfavorably, but generally it can be assumed that although the signs of each term may be plus or minus, the summation forming $\mathrm{q}(\mathrm{z})$ will be at least close to having $\varepsilon$ correct decimal places)。

Since the computer carries only eight significant figures in all computations, less than $\varepsilon$ of these eight digits will be to the right of the decimal 
point if the magnitude of a given term in Eq. (4) is too large. When the codei: " detects a potentially excessive round-off error, a decision is made to avoid $i t$ : by actually treating $t+1$ collisions analytically instead of the $p+1$ collisions for which $\alpha_{i}$ and $\beta_{i}$ were calculated in Eqs. (45). Since each of the exponentials multiplying the $\mathrm{D}_{\mathrm{k}}^{i{ }^{\mathrm{s}} \mathrm{s}}$ in Eq. (4) is not greater than unity, each term will have at least $\varepsilon$ decimal place accuracy if the coefficient $D_{k}^{i}$ itself has this accuracy。 Therefore., EURIPUS goes through the following procedure: immediately after it is calculated, each $D_{k}^{i}$, for $i>0$, is tested to see if

$$
\left|D_{k}^{i}\right|<10^{8-\varepsilon}
$$

If so, its first $\varepsilon$ decimal places will be accurate, and the recurrence calculation may proceed; if Eq. (50) is not satisfied, $t$ is set equal to $i-I_{\text {, and no }}$ more $\mathrm{D}_{\mathrm{k}}^{i}$ 's are calculated. If Eq. (50) is satisfied for all $i \leq p$, the code sets $t=p$ and stores only the quantities $D_{k}^{t}$, with $k=0$ thru $t$.

Equation (50) is applied only for $i>0$ since the spatial distribution of at least one collision must be treated analytically in order to obtain a density function. In this case, with $i=t=0$, only one term in Eq. (4) results, and although it may. not have $\varepsilon$ decimal places accurate, this estimate of $q(z)$ will be correct to eight significant figures, since no subtractions are involved. Ihus, the output for $q(z)$ will be an average of values that are accurate either to eight significant figures or else $\varepsilon$ decimal places. Therefore, the output itself will have $\varepsilon$ decimal place accuracy unless the answer is so large that such accuracy would imply more than eight significant figures: in the latter circumstance, eight significant figure accuracy results.

Because the factors multiplying $P_{R}(z)$ in Eqs. (15), (19), and (20). change with each history, the above procedure guaranteeing a certain numerical accuracy in $P_{R}(z)$ would not guarantee a definite numerical accuracy in $\varphi(z)$. 22 
However, if $D_{0}^{0}$ is multiplied by these factors and the recursion formalas of Eqa" (5) are applied, an algebraic expression is obtained for $\mathrm{P}_{\mathrm{R}}(\mathrm{z})$ which is identical to that for $\varphi(z)$, and checking for round-off error at each recursion"giarantees the same sort of accuracy for $\varphi(z)$ as we had before for $q(z)$. Furthermore, the black foil activation in Eq. (16) can be guaranteed"to have the same accuracy "since the factor $\left|\mu_{R}\right| \leqslant 1_{\text {. }}$ The extra function in Eq。 (17), obtained by linearly interpolating for $F\left(\mu_{R}\right)$ between tabulated values, cannot always be guaranteed such accuracy because, unlike $\varphi(z), q(z)$; and black foil activation, the values it averages over may be plus or minus; however, if there are few histories which have eight digit accuracy, instead of $\epsilon$ decimal place accuracy; the final average will have $\epsilon$ digit accuracy。

Unlike EURIPUS, the factors of $P_{R}(z)$ in DAEDALUS mast be obtained: by following the neutron to an energy below $E_{R}$ before the coefficients $D_{k}^{i}$ can be obtained from Eq. (5)。 of the several possibilities for handling split collisions in DAEDALUS, a simple procedure was chosen which may result in extra Monte cario spatial calculations; since usually few histories will be split and since usually round-off error will already have necessitated Monte Carlo replacing analytic calcalations by the time"splitting starts; the procedure is "considered" satisfactory. For each part of the split there are two factors which should multiply $D_{0}^{0}$; they are, from Eqs。(15), (19), and (20):

$$
x_{1}=\frac{1}{\sum_{R}^{S}(I-\alpha) E_{j} / E_{R}} \quad \text { and } \quad x_{2}=\frac{I}{\sum_{R}^{S}\left(I-\alpha \cdot E_{I-1} / E_{R}\right)} \text {, }
$$

where $\alpha$ in Eq. (5I) equals zero if the last colliston was with hydrogen。 $D_{0}^{0}$ is multiplied by $\bar{x}$, the larger of $x_{1}$ and $x_{2}$ and the recursions of Eq. (5) 
are performed until i reaches either $t$ for the part of the split whose $x=x^{3}$ else 1 equals $r$ for the other part of the gplit。' If $x$, the amaller $x$ had been:. used to maltiply $D_{0}^{0}$, the recurstions would not have been terminated for any angler value of 1 . Therefore without fear of round-off error, we can watiply $p_{k}^{l}$ for the $i$ terminating the recursions, by $x / x$ to obtiain the $p_{k}^{1}$ for the other part of the split. While it would be possible to continue the recursions for these smaller $R_{k}^{1}$, DAEDALUS stops the recursion formalas for simplicity at this point and allows the rest of the spatial distribution to be calculated by analog Monte Carlo.

The next step is to calculate, by stratght analog Monte Carlo, the quantities $w_{t p}$ and $z_{t p}$ of $E q_{0}(6)$. If $t \neq p$,

$$
w_{t p}=\prod_{1=t+1}^{p} \alpha_{1} / \beta_{1}
$$

and

$$
z_{t p}=z_{1}-\sum_{i=t+1}^{p}\left(\ln \xi_{i}\right) / \beta_{1}
$$

where the $\xi_{1}{ }^{0}$ are $p-t$ different random numbers. If $t=p_{,} w_{t p}=1$ and $\mathbf{z}_{\mathrm{tp}}=\mathbf{z}_{\mathrm{T}^{\circ}}$

We have now accounted for all $p$ of the collialons that would have been treated analytically if there were no round-off error。 However, if $N<r_{q}$ there are still $r-N$ collisions left to treat before the history is ended at $1=r_{g}$ and these are calculiated by analog Monte Carlos

$$
w_{t 1}=w_{t i-1} \Sigma_{1}^{S} / \Sigma_{1}^{T}
$$

and

$$
z_{t 1}=z_{t 1-1}-\left(\mu_{1} / \Sigma_{1}^{T}\right) \ln \xi_{1} \text { for } 1=p+1 \text { thru } r
$$




$$
\mathrm{N} \geq r, w_{t r}=w_{t p}: \text { and } z_{t r}=z_{t p}
$$

Equations (52) and (53) are applied at each collision by including them in a calculation using the relevant parts of Eqs. (49).

The point has been reached for the evaluation of Eq. (6), using Eq. (4). Now, it is a very time consuming procedure to calculate an exponential for each value of $z$ inputted, for each collision of each history; in fact such excessive computing time provides the main reason why it is more economical to allow the spatial calculations of some collisions to be performed by Monte Carlo, as in Eq. (55), rather than analytically even when there is little round-off error. However, experience with EURIPUS-2 has shown that many of the terms contributing to the analytic expression for the slowing down density are very small. Much time can be saved by not evaluating any exponentials whose net effect is smaller than the error already accepted from round-off. Thus the exponentials in Eq. (4) are replaced by the functions $f_{k}(z)$, to be defined below.

$$
\begin{aligned}
& \text { Then, for } z \geq z_{t r}, \\
& \qquad P_{r}(z)=w_{\operatorname{tr}} \sum_{\substack{k=0 \\
\left(\beta_{k}+\right)}}^{t} D_{k}^{t} f_{k}(z),
\end{aligned}
$$

where the sum is over terms $\mathrm{k}$ for which $\beta_{\mathrm{k}} \geq 0$. For $z<\mathrm{z}_{\mathrm{tr}}$,

$$
P_{r}(z)=-w_{t r} \sum_{\substack{k=0 \\\left(\beta_{k}-\right)}}^{t} D_{k}^{t} f_{k}(z)
$$

where the sum is over terms $\mathrm{k}$ for which $\beta_{\mathrm{k}}<0$.

$$
\begin{array}{rlrl}
f_{k}(z) & =\exp -\beta_{k}\left(z-z_{t r}\right) & & \text { if } \beta_{k}\left(z-z_{t r}\right)<18.5 \\
& =0 & \text { if } \beta_{k}\left(z-z_{t r}\right) \geq 18.5 .
\end{array}
$$


The second part of $\mathrm{Eq}$. (59) states that zero replaces the exponential when the latter is less than $e^{-18.5} \approx 10^{-8}$. According to Eq。 $(50)$, the product of $D_{k}^{t}$ and an exponential approximated by zero is less than $10^{-6} ; i_{\circ} e_{0}$ only those dectmal places beyond the $\epsilon$-th will be affected, and these were already accepted as being in error.

If $B_{k}^{t}$ were afficiently small, $f_{k}(z)$ for all $z$, could be set equal to zero, and Eq. (59) would not even have to be applied at each space point zo Since $f_{k}(z) \leqslant i, D_{k}^{t}$ could be set equal to zero if its absolute value were less than $10^{-6}$. In fact, however, $f_{k}(z)$ and $D_{k}^{t}$ are set equal to zero if

$$
\left|p_{k}^{t}\right| \leqslant 10^{-\epsilon-2} \text {, }
$$

a criterion which accepts only errors 100 times as small as the round off error. This safety margin was taken because the $\mathrm{D}_{k}^{t_{p}}$ s also enter the calculation of the moments, whose round off error (as for all quantities in most codes) is not pre: cisely known but is expected to have roughly the same percentage as the denfity. functions, whose round off error is controlled. Although the round off error in the moments is not precisely controlled, confidence in their means maj still be measured by the variances, which include fluctuations due to round off errors。 We now come to the calcalation of the spatial moments of the density functions. Except for certain zero moment $(K=0)$ quantities, the values of which cen be shown fron physical considerations to be bounded, for each $k$ the calculated functions are checked to see if thielr values would be too amall or too large for the computing pachine. A number that would cause an underflow is. sept equal to zero, and there is an error stop if any mumer overflows。

The full range zeroth moments of flux or slowing down density are just the infinite medium values, independent of spatial effects, so $v_{k}^{\mathrm{K}}$ in $\mathrm{Eq}$ (26) 1. tested for under and overflow only for $K>0$ o $D_{k}^{t} v_{k}^{K}$ is tested for underflow 26 
for all $K$, and there is formed

$$
v^{K}=w_{t r} \sum_{k=0}^{t} D_{k}^{t} v_{k}^{K},
$$

of which the square is tested for over and underflow and the sums of squares over all histories are tested for overflow for $K>0 .\left|\mu^{R} V^{K}\right|^{2}$, and $F\left(\mu_{R}\right) \nu^{K}$, used in calculating moments in DAEDALUS, is also checked for underflow. If $\beta_{\mathrm{k}} z_{\mathrm{tr}}<-88$ in Eq. (25), $\mathrm{U}_{\mathrm{k}}^{\mathrm{O}}$ is set equal to zero. From this point on, the calculation and testing of quantities leading to $\tau^{\mathrm{K}}$, and related quantities is exäcty the same as for $v^{K}$ described by Eq. (6I).

Besides the density functions, their moments, and the probable (50\%) errors, the codes output certain quantities which are of interest in controlling the manner of calculation. The average s andits:probable error are outputted and labeled "monte carlo position calculations." The average $r$ and its probable error are also outputted in EURIPUS and labeled "collisions-1." "This number is one less than the average number of collisions a source neutron takes, to slow down past $E_{R^{\circ}}$ In DAEDALUS, the average value of $r$ and its probable error are also outputted and labeled "collisions." This number is also the average number of collisions for a neutron to slow down past $E_{R}$, even though the code calculates densities in $d u_{R^{\circ}}$ The average $r$ is one larger in EURIPUS than in DAEDALUS because of Eq. (47). Even when $N=0$, one collision is done analytically, so that "collisions" equals "Monte Carlo position calculations" in this case.

When splitting occurs in DAEDALUS, each split has a different number of collisions. Therefore the $r$ for the history has the correctly weighted mean,

$$
\mathrm{r}=\mathrm{yr}_{2}+(1-\mathrm{y})(\mathrm{j}+1),
$$


where $E_{j}$ is the energy where the split occurs according to Eq. (18), $y$ is the fraction that have collisions above $E_{R}$ after collision $j$ according to Eq. (20), and $r_{2}+1$ is this number of collisions. Finally, the net number of histories where splitting occurs is printed out.

\section{REFERENCES}

1. DAEDALUS was formulated especially to attempt to reproduce the experimental reoults of Jo DeJuren, M. Heier, and R. Stookoberry, WAPD.'l' 1058 (to be published), where Indium foils in a large tank of water were activated by neutrons from a D-D source fed in through a tube.

2. The code held most specifically in mind is RDR-5, developed by E. Bareiss, C. 'Dawson, and D. Schiff, and not yet described in a written report. The vector flux is approximated by ten Legendre polynomials for its angular dependence and by straight lines between groups for its lethargy dependence.

3. M. Jo Berger and J. Dogett, Jour。Res. Natl. Bur.Stands, Vol. 56, No. 2 (1956).

4. D. W. Drawbaugh, "Stochastic Estimation of the Neumann Series Using Conditional Monte Carlo," Combustion Engineering Inc. Report TP-19 (1958)。

5. S. Glasstone and M. C. Edlund, The Elements of Nuclear Reactor Theory,

(D. Ven Nogtrand Co., New York).

6. R. E. Marshak, Revs. Mod. Fhys. 19, 185 (1947).

7. P./F. Zweifel and H. Hurwitz, Jr., J. Appl. Phys. 25, 1241 (1954). 


\title{
PART II。 STATISTICAL ANALYSIS
}

\author{
Jerome Spanier
}

For completeness a brief description of the statistical analysis for EURIPUS and DAEDALUS is presented here. 'This analysis is straightforward, being based only. on the Central I.imit Theorem of probability theory, and applies equally well. to any of the parameters being estimated by the programs.

Let :C.denote an arbitrary neutron history in EURIPUS or DAEDALUS: that is, a sequence of points in phase space corresponding to the random : walk made by the particle. The totality of all such histories will be denoted by the symbol $\Omega$. The space $\Omega$ is endowed with a natural probability measure $\mathrm{P}(\mathrm{C})$, the ànalog measure (see Ref。 $I$, $\$ 8$, for a complete definition) which reflects the likelihood of occurrence of specific sets of histories according to the physical model postulated. For each unknown quantity being estimated a random variable $\xi$ is defined ${ }^{\star}$. This random variable is a real-valued function on the space $\Omega$ which measures the contribution of each history to the overall estimate of the unknown parameter. If the random variable is unbiased, its expected value, defined as

$$
E[\xi]=\int_{\Omega} \xi(C) d P(C)
$$

where $d P(C)$ is the differential of the measure $P(C)$, is equal to the parameter being estimated. The assumption is now made that all random variables dealt with are unbiased. Let. $\mathrm{m}=\mathrm{E}[\xi]$ denote the desired expected value and let

*The basic random variable of DAEDALUS, $\xi_{F}$ is defined precisely in Part IV and shown to be unbiased there. 


$$
\begin{gathered}
\sigma^{2}=E\left[\xi^{2}\right]-\dot{E}^{2} \cdot[\xi] \\
=\int_{\Omega} \xi^{2}(c) d P(C)-\left[\int_{\Omega} \xi(C) d P(C)\right]^{2}
\end{gathered}
$$

denote the unknown variance of $\xi$.

In general $K$ histories, $C_{1}, \ldots, C_{K}$ àre generated in sücccession. The $K$ numbers, $\xi\left(c_{1}\right), \cdots, \xi\left(C_{K}\right)$ represent the totality of information available for estimating $\mathrm{m}$ and for predicting the statistical uncertainty assôciated with the estimate. The theory which makes this possible will now be skettched.

Random variable es $\xi_{i}$ are defined on the space of $k$ - tiüpìies of histories, denoted $\Omega^{\mathrm{K}}$, by

$$
\xi_{i}\left(c_{1}, \cdots, c_{K}\right) \equiv \xi\left(c_{i}\right), i=1, \cdots, K
$$

In addition, define a random variable $\bar{\xi}$ by

$$
\bar{\xi}=\frac{1}{K} \sum_{i=1}^{K} \xi_{i}
$$

so that $\bar{\xi}$ is a random variable on the space $\Omega^{K}$ whose value on the $K$-tuple $\left(c_{1}, \cdots, c_{K}\right)$ is the average weight of these $K$ histories,

$$
\bar{\xi}\left(c_{1}, \cdots, c_{K}\right)=\frac{1}{K} \sum_{i=1}^{K} \xi\left(c_{i}\right)
$$

It seems intuitively reasonable that for sufficiently large $\ddot{K}$, the expression (5) provides a good approximation to $m_{\text {. Th }}$ This made precise by the following results 


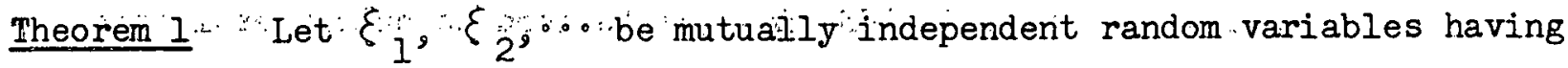
a common distribution with mean $m$ and (finite) variance $\sigma^{2}$. Then the random variable $\bar{\xi}=\frac{1}{K} \sum_{i=1}^{K} \xi_{i}$ is asymptotically (for large $K$ ) normally distributed with expected value $m$ and variance $\sigma_{K}^{2}=\frac{\sigma^{2}}{K}$. For a discussion of results relating to this see for example Ref。2, pp. $213 f f$.

For the purposes of the statistical analysis the foregoing theorem is translated into the following formula:

For any $\varepsilon>0$,

$$
P\left\{\left(c_{1}, \cdots, c_{K}\right) \varepsilon \Omega^{K}:\left|\bar{\xi}\left(c_{1}, \cdots, c_{K}\right)-m\right|<\varepsilon\right\} \sim \sqrt{\frac{2}{\pi}} \int_{0}^{\frac{\varepsilon}{\sigma_{K}}} e^{-t^{2} / 2} d t,
$$

where $\sigma_{K}=\sqrt{\sigma_{K}^{2}}$. In words, formula (6) states that the probability that $K$ histories $C_{1}, \cdots, C_{K}$ will be selected whose average weight differs from the true mean $m$ by an amount less than $\varepsilon$ is approximately the integral on the right. Since $\sigma_{K}$ approaches $O$ as $K$ tends to infinity, the integral approaches $I$ as $K$ tends to infinity, for each fixed $\varepsilon_{0}$;This guarantees that $\bar{\xi}$ tends to $m$ in the measure-theoretic sense of Eq. (6).

Of course the above analysis depends upon obtaining estimates of the quantity $\sigma_{K}$ by some means, as this is also unknown in practice. One ordinarily chooses

$$
S^{2}=\frac{K}{K-1}\left[\frac{1}{K} \sum_{i=1}^{K} \xi^{2}\left(c_{i}\right)-\left(\frac{1}{K} \sum_{i=1}^{K} \xi\left(c_{i}\right)\right)^{2}\right]
$$

as an estimate of the variance $\sigma^{2}$ and then $\sqrt{\frac{S^{2}}{K}}$ is used to estimate $\sigma_{K}$ for the purpose of Eq. (6)。 
In both EURIPUS and DAEDAIUS, Eq. (6) is used to obtain estimates of the probable error, i.e., the size of the $50 \%$ confidence intervals. Using the fact that

$$
\sqrt{\frac{2}{\pi}} \int_{0}^{.6745} e^{-t^{2} / 2} d t=.5
$$

one solves for $\varepsilon$ in Eq. (6) as a function of the sample estimate of the variance:

$$
\varepsilon=(.6745) \sqrt{\frac{\mathrm{S}^{2}}{K}}
$$

The $\varepsilon$ so obtained is the probable error, which implies that the interval $\bar{\xi}\left(C_{1}, \cdots, c_{K}\right) \pm \varepsilon$ will contain the true mean $m$ about $50 \%$ of the time.

\section{References}

1. J. Spanier, "Monte Carlo Methods and Their Application to Neutron Transport Problems," WAPD-195 (July 1959).

2. H. Cramer, "Mathematical Methods of Statistics," Princeton Universi.ty Press, 1946. 


\section{PART III: EXAMPLES TESTING FORMULATION AND ACCURACY}

Harvey J. Amster

\section{INTRODUCTION}

The principles of these Monte Carlo Density codes, as described in Parts I and II, seem complex enough to warrant further evidence for their validity. In Part IV, a formal mathematical discussion of the theory is given. In this section, however, we shall make an experimental test of the validity of the codes by comparing some of their results with those of analytic calculations for special simple cases. These sample problems also test the assumptions controlling round-off error and degenerate histories. In addition, problems with different control parameters will demonstrate the advantages in doing parts of the calculation analytically。

We will now derive some rigorous expressions for the case when only one moderator, with mass A, is present. Its scattering cross section is constant in energy and isotropic in the C.Mo system. There is no absorption, and the source is isotropic and monoenergetic with $E_{0}<E_{R} / \alpha$ 。

The full range moments; will be shown to require solutions of the general form,

$$
y(u)=A \int_{0}^{u} d u^{\prime} y\left(u^{\prime}\right) \cdot e^{a\left(u-u^{\gamma}\right)}+B \int_{0}^{u} d u^{p} y\left(u^{\prime}\right) \cdot e^{b\left(u-u^{\gamma}\right)}+e^{c u}
$$

Let

$$
I_{a}(u) \equiv A: \int_{0}^{u} d u^{\prime} y\left(u^{\prime}\right) e^{a\left(u-u^{\prime}\right)}
$$

and similarly with $I_{b}(u)$. Then

$$
I_{a}^{\prime}=A y+a I_{a} \text { 。 }
$$


Equation (I) and its first two derivatives can thus be written:

$$
\begin{aligned}
& e^{c u}+I_{a}+I_{b}=y \\
& c e^{c u}+a I_{a}+b I_{b}=y^{\prime}-(A+B) y \\
& c^{2} e^{c a}+a^{2} I_{a}+b^{2} I_{b}=y^{\prime \prime}-(A+B) y^{\prime}-(a A+b B) y .
\end{aligned}
$$

Consider Eqs. (4) as three linear equations in the three unknowns, $e^{\text {cu }}, I_{a}, I_{b}$ ? and solve for the first。

$$
e^{c i 1}\left|\begin{array}{lll}
1 & 1 & 1 \\
c & a & b \\
c^{2} & a^{2} & b^{2}
\end{array}\right|=\left|\begin{array}{lll}
y & 1 & 1 \\
y^{3}-(A+B) y & a & b \\
y n^{n}-(A+B) y^{n}-(a A+b B) y & a^{2} & b^{2}
\end{array}\right|
$$

Since determinants are zero if two columins are equal, the coefficient of $e^{\text {cu }}$ is $(b-a)(c-a)(c-b)$. The right side of Eq. (5) also contains the factor $(b-a)$, which can be separated by subtracting the third column from the second. This factor can then be removed from both sides of the equation and the determinants expanded to give simply

$$
y^{\prime \prime}-2 F y^{j}+G y=(c-a)(c-b) e^{c d},
$$

where

$$
F \equiv \frac{l}{2}(A+B+a+b) \text { and } G \equiv a b+a B+A b
$$

By standard means, the solution is

$$
\mathrm{y}=\mathrm{He} \mathrm{e}^{\mathrm{cu}}+\mathrm{K}_{+} e^{\lambda_{+} \mathrm{u}}+\mathrm{K}_{-} \mathrm{e}^{\lambda \mathrm{u}^{\prime}}
$$

where

$$
H=(c-a)(c-b) /\left(c^{2}-2 F c+G\right)
$$

and

$$
\lambda_{ \pm}=F \pm \sqrt{F^{2}-G} \text { 。 }
$$


$\mathrm{K}_{ \pm}$are determined by substituting $\mathrm{Eq},(7)$ into $\mathrm{Eq},(1)$ and equating to zero the coefficients of $e^{a u}$ and $e^{\text {bu }}$ that occur on the right side:

$$
K_{ \pm}=\frac{\left(\lambda_{ \pm}-a\right)\left(\lambda_{ \pm}-b\right)}{c^{2}-2 F c+G} \frac{\left(\lambda_{I}-b\right)(c-a)-\left(\lambda_{\mp}-a\right)(c-b)}{\left(\lambda_{I}-b\right)\left(\lambda_{F}-a\right)-\left(\lambda_{I}-a\right)\left(\lambda_{F}-b\right)}
$$

In particular, if $B=0$,

$$
y=\frac{1}{a+A-c}\left[(a-c) e^{c u}+A e^{(a+A) u}\right]
$$

unless $a+A=c$, in which case the limit holds:

$$
y=(A u+1) e^{c u}
$$

We will make use of this solution to Eq. (1) by noting that if the exponential in Eq. (1). is replaced by a linear combination of exponentials, the solution will be the same linear combination of solutions for each exponential alone.

With the lethargy defined to be zero at the source, Eqs. (105) of Marshak (Ref。6) can be-generelized to indude non-hydrogenous moderators in our situation:

$$
\begin{aligned}
& x_{00}(u)=\int_{0}^{u} \min ^{p} f_{0}\left(u, u^{\beta}\right) x_{00}\left(u^{8}\right)+f_{0}(u, 0) . \\
& x_{11}(u)=\int_{0}^{u} d u^{8} f_{1}\left(u, u^{8}\right) x_{11}\left(u^{p}\right)+\frac{\ell}{3}\left[f_{1}(u, 0)+x_{00}(u)\right] \\
& x_{02}(u)=\int_{0}^{u} d u^{0} f_{0}\left(u, u^{0}\right) x_{02}(u !)+\frac{2 \ell^{2}}{3} f_{0}(u, 0)+2 k x_{u_{1}}(u) \ldots
\end{aligned}
$$

$\ell$ is the reciprocal of the macroscopic total (or scattering) crass section. $l x_{00}$ and $l x_{02}$ are the zeroth and second fuli range moments of the flux density, 
and $l X_{1 I}(u)$ is the first moment of the current, evaluated at the lethargy $u$. For an element of arbitrary mass (Ref。7),

$$
\begin{aligned}
& f_{0}\left(u, u^{\prime}\right)=\frac{1}{1-\alpha} e^{-\left(u-u^{\prime}\right)} \quad \text { and } \\
& f_{1}\left(u, u^{p}\right)=\frac{1}{(1-\alpha)\left(1-\alpha^{1 / 2}\right)}\left[e^{-\frac{3}{2}\left(u-u^{p}\right)} \cdot a^{\frac{1}{2}} e^{-\frac{1}{2}\left(u-u^{0}\right)}\right]
\end{aligned}
$$

Equations (12) can be solved in order from solutions of the form of Eq. (I), since the exponential "source" of the latter yield exponential solutions, which act as new sources for the following integral equation (12).

As it turms out, $G>F^{2}$ in some cases, and $\lambda \pm$ in Eq. ('9) are complex numbers. However, one can readily see that since $\lambda_{ \pm}$are complex conjugates, $\mathrm{K} \pm$ in Eq. (10) are also complex conjugates, and therefore $\mathrm{y}$ in Eq. (7) is real. The final results are

$$
\begin{aligned}
& x_{00}=\frac{1}{1-\alpha} e^{\frac{\alpha u}{1-\alpha}} \\
& x_{11}=\frac{\alpha}{3(1-\alpha)} \sum_{i=1}^{3} D_{i}\left[H_{i} e^{c_{i} u}+K_{i+} e^{\lambda_{+} u}+K_{i-} e^{\lambda_{-} u}\right]
\end{aligned}
$$

where $\lambda_{ \pm}$come from Eqs. (.6) and (9) with

$$
a=-3 / 2 ; \quad b=-1 / 2 ; \quad A=1 /(1-a)\left(1-a^{1 / 2}\right) ; \quad B=-\alpha^{1 / 2} A
$$

and $\bar{H}_{i}, \mathrm{~K}_{i+}$, and $\mathrm{K}_{i_{-}}$come from Eqs. (8) and (10) with the help of Eq. (17) and

$$
c_{1}=\alpha /(1-\alpha) ; c_{2}=-3 / 2 ; c_{3}=-1 / 2
$$

The only other quantities needed in Eq. (16) are

$$
D_{1}=1 ; D_{2}=1 /\left(1-\alpha^{1 / 2}\right) ; D_{3}=-a^{1 / 2} /\left(1-a^{1 / 2}\right)
$$


Finally,

$$
\begin{aligned}
& x_{02}=\frac{2}{3} l^{2}\left\{x_{00}+\sum_{i=1}^{3} \frac{D_{i} H_{1}}{a-(1-a) c_{i}}\left[x_{00}-\left(1+c_{i}\right) e^{c_{i} u}\right]\right. \\
& +\left[x_{00}-\left(1+\lambda_{+}\right) e^{\lambda_{+} u}\right]\left[a-(1-a) \lambda_{+}\right]^{-1} \cdot \sum_{i=1}^{3} D_{i} K_{i+} \\
& +\left[x_{00}-\left(1+\lambda_{-}\right) e^{\lambda_{-}^{u}}\right]\left[a-(1-\alpha) \lambda_{-}\right]^{-1} \cdot \sum_{i=1}^{3} D_{i} K_{i-}
\end{aligned}
$$

It is also possible to calculate $\mathrm{P}_{\mathrm{r}}(\mathrm{E})$, the probability that it will take $r$ collisions for a neutron with energy $E$ to reach an energy below $E_{R^{*}}$ From the physical situation, we find immediately that

$$
P_{1}(E)=\left(E_{R}-\alpha \bar{E}\right) /(1-\alpha) E
$$

and for higher values of $r$,

$$
P_{r+1}(E)=\frac{1}{(1-\alpha) E} \int_{E_{R}}^{E} P_{r}\left(E^{q}\right) d E^{p}
$$

By induction,

$$
P_{r+1}(E)=\frac{1}{(1-\alpha)^{r+1}}\left[\left(\frac{E_{R}}{E}\right)\left(\frac{u^{r}}{r i}+\alpha \sum_{i=0}^{r-1} \frac{u^{i}}{i !}\right)-a\right],
$$

where

$$
u \equiv \ln \left(\mathrm{E} / \mathrm{E}_{\mathrm{R}}\right)
$$

With mach manipulation, we can evaluate the sum

$$
\bar{r}=\sum_{r=1}^{\infty} r P_{r}=\frac{1}{\alpha}\left[\left(\frac{E}{E_{R}}\right)^{\alpha /(1-\alpha)}-1\right]+1
$$


which agrees with the intuitive result,

$$
\bar{r}=1+\int_{E_{R}}^{E} \Sigma_{S}\left(E_{R}^{r}\right) \varphi\left(E_{R}^{q}\right) d E_{R}^{r}
$$

Incidentally, since Eq. (26) is true in general, and since EURTPUS-3 and DAEDALUS always output the average number of colliṣions per history, one can obtain from the codes estimates of integrals of the zeroth moments of the scattering collision dénsity.

Equation (23) can also be manipulated to yield

$$
\overline{r^{2}}=\sum_{r=1}^{\infty} r^{2} P_{r}=1-\frac{3}{\alpha}+\frac{2}{\alpha^{2}}+\frac{1}{(1-\alpha)}\left(\frac{E}{E_{R}}\right)^{\alpha /(\alpha-1)}\left[\frac{2}{\alpha} u-3+\frac{5}{\alpha}-\frac{2}{\alpha^{2}}\right] .
$$

Thus, the expected value of the probable error after $N_{H}$ histories can be obtained from Eqs. (25) and (27):

$$
P . E_{0}=0.6745 \sqrt{\frac{\overline{r^{2}}-(\bar{r})^{2}}{N_{H}-1}}
$$

Equation (28) is valid only when no histories are split, einco the analytic lieatment effectively increases $\mathrm{N}_{\mathrm{H}}$ 。

II. The First Sample Problem

With $\mathrm{A}=16, \mathrm{E}_{\mathrm{O}}=1.25 ; \mathrm{E}_{\mathrm{R}}=1, \dot{\Sigma}^{\mathrm{IM}}=1.5$, Eqs. (1) through (28) were evaluated after an extremely long numerical calculation. In addition to these parameters being fed into DAEDALUS, $\varepsilon=4, \rho=\ell=0$, and $F\left(\mu_{R}\right)=\mu_{H}$, making the extra output-function current. Three separate muns were made, each with different control parameters: Runs $I$ and 2 had $N=0$, givịng each history always just one analytical spatial calculation; however, the splitting parameters were such that no history split in the first mu, while all histories split in the second. Run 3 had no splitting, but $\mathrm{N}=50$ so that nearly all the spatial calculations were done analytically. The following table summarizes most of the results. 
FIRST SAMPLE PROBLEM $(\mathrm{A}=16)$ RFSULTS

Quoted Errors are Probable (50\%) Errors

Run:

Histories:

Analytic Spatial Calculations per History:

Split Histories: $\begin{array}{cc}1 & 2 \\ 5000 & 5000\end{array}$

$\cdot 1$

1

None

All
5000

Almost All

None

Full Range Moments, Exact Analytic

of. Flux

$$
\begin{aligned}
& 0 \\
& 1 \\
& 2
\end{aligned}
$$

Full Range Moments of Current

$\begin{array}{ll}0 & 0 \\ 1 & 1.204 \\ 2 & 0\end{array}$

Average No. of Collisions: Measured Prob. Error:

Exact Prob. Error:

Continuing with Run: Histories:

Full Range Moments of Flux

0
1
2

Full Range Moments of Current

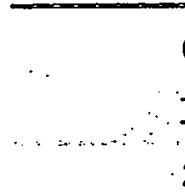

$\begin{array}{ll}0 & 0 \\ 1 & 1.204 \\ 2 & 0\end{array}$

Average No. of Collisions:

Measured Prob. Error:

Exact Prob. Error:

2.5301

6.596

0

6.393
Results

$\begin{array}{lrllll}6.596 & 6.63 & \pm 0.06 & 6.57 & \pm 0.02 & 6.56 \pm 0.06 \\ 0 & -0.05 & \pm 0.06 & 0.01 & \pm 0.05 & 0.02 \pm 0.04 \\ 6.393 & 6.50 & \pm 0.11 & 6.43 & \pm 0.10 & 6.54 \pm 0.06\end{array}$

2.5301

2.5220

2.5358

2.5446

0.009201

0.0075

0.009214

0.009199

0.009199

20,000

$2^{3}, 000$ $4^{3}, 000$

$0.027 \pm 0.05$

$0.08 \pm 0.03$

$0.07 \pm 0.05$

$1.191 \pm 0.04 \quad 1.24 \quad \pm 0.03$

$1.23 \pm 0.02$

$-0.26 \pm 0.09$

$0.09 \pm 0.06$

$\begin{array}{rrrrr}6.61 & \pm 0.03 & 6.61 & \pm 0.03 & 6.60 \pm 0.02 \\ 0.01 & \pm 0.03 & -0.03 & \pm 0.02 & -0.00 \pm 0.02 \\ 6.39 & \pm 0.06 & 6.47 & \pm 0.03 & 6.44 \pm 0.02\end{array}$

$6.39 . \pm 0.06$

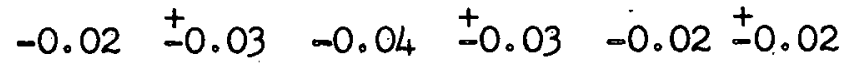

$\begin{array}{lllll}1.19 & \pm 0.02 & 1.21 & \pm 0.01 & 1.20 \pm 0.01\end{array}$

$-0.11 \quad \pm 0.04 \quad-0.02 \quad \pm 0.03 \quad-0.01 \pm 0.02$
2.5199
2.5360
2.5371
0.0046
0.0046
0.0033
0.0046
0.0046
0.0033 
The comparative accyracy of the three mins should be judged on the basis of measured probable errors rather than actual deviations from analytic results, which are largely a matter of luck for a given experiment and would be sensitive to the sequence of random numbers used.

The relative accuracies after 5000 histories can be interpreted:

since Runs $I$ and 3 differ only in the number of analytic spatial calculations, their zeroth moments and average numbers of collisions are equally accurate. In Run 2, however, the splitting has the effect of doing more of the energy distribution analytically and improves accuracy. For the higher spatial moments, Run 2 is better than Fun 1 because of the splitting, and kun 3 is better than fun $I_{8}$ since the only difference is that fun 3 does more of the spatial calculation analytically。

Actual deviations from the exact answers, when compared with the probable errors, can be used to gain confidence in the reliability of the code. Although most quantities seem reasonably accurate after 5000 histories, the second moment of the current in Run 1 and the second moment of the flux in fun 3 looked suspicious enough to check for nonstatistical errors by running more histories. While the deviations remain large relative to their new probable errors, the latter have become smaller, making the new estimates closer to the analytic results. The chance that the second moment of the:fiux in frun 3. after 40,000 histories could be worse is $20 \%$; the number of collisions, 15\%. Since. these errors are not terribly unlikely and correspond to actual deviations less than 1\%, and since other quantities came out more reasonably, the test problem is considered a success. 


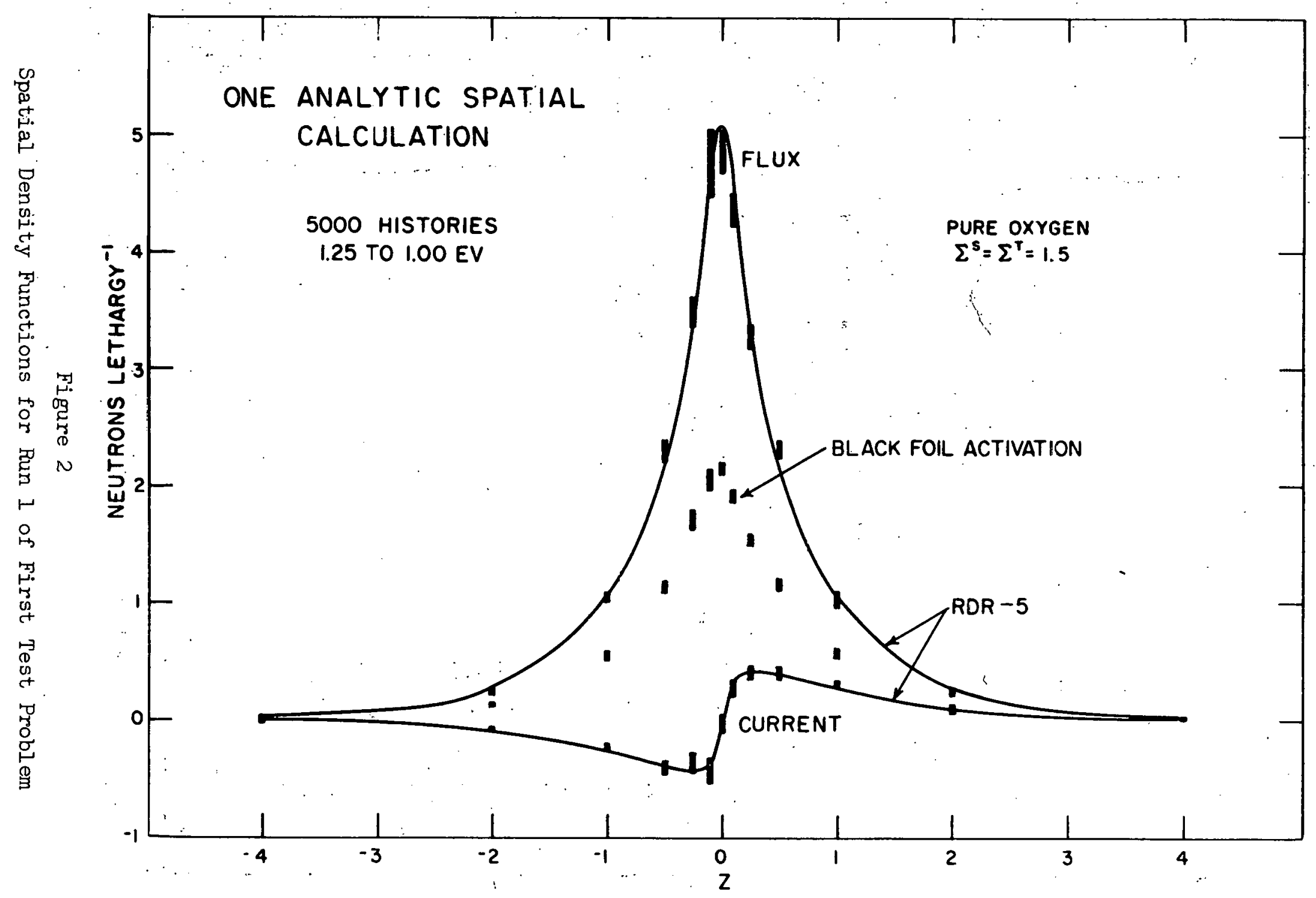


交

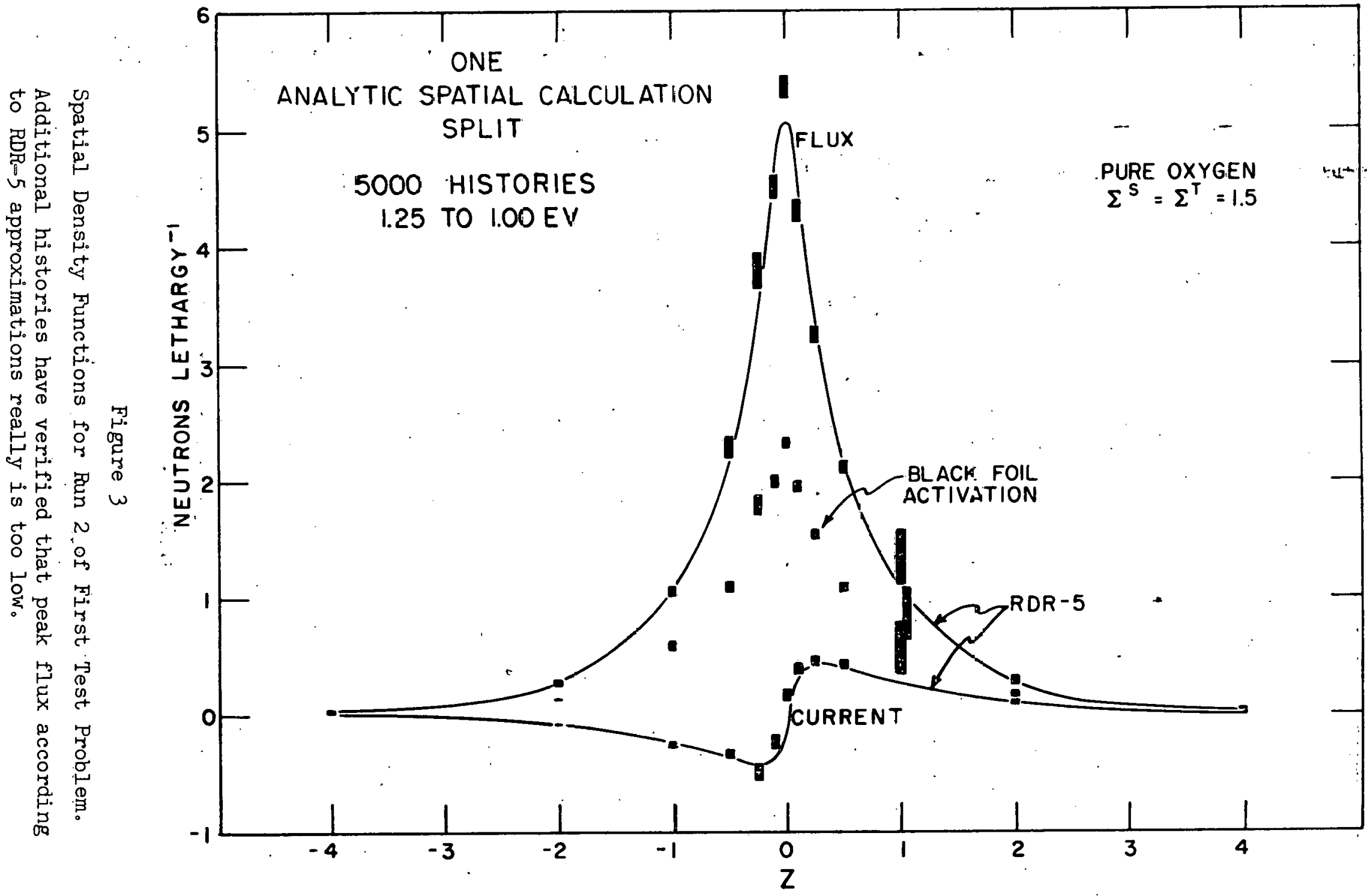




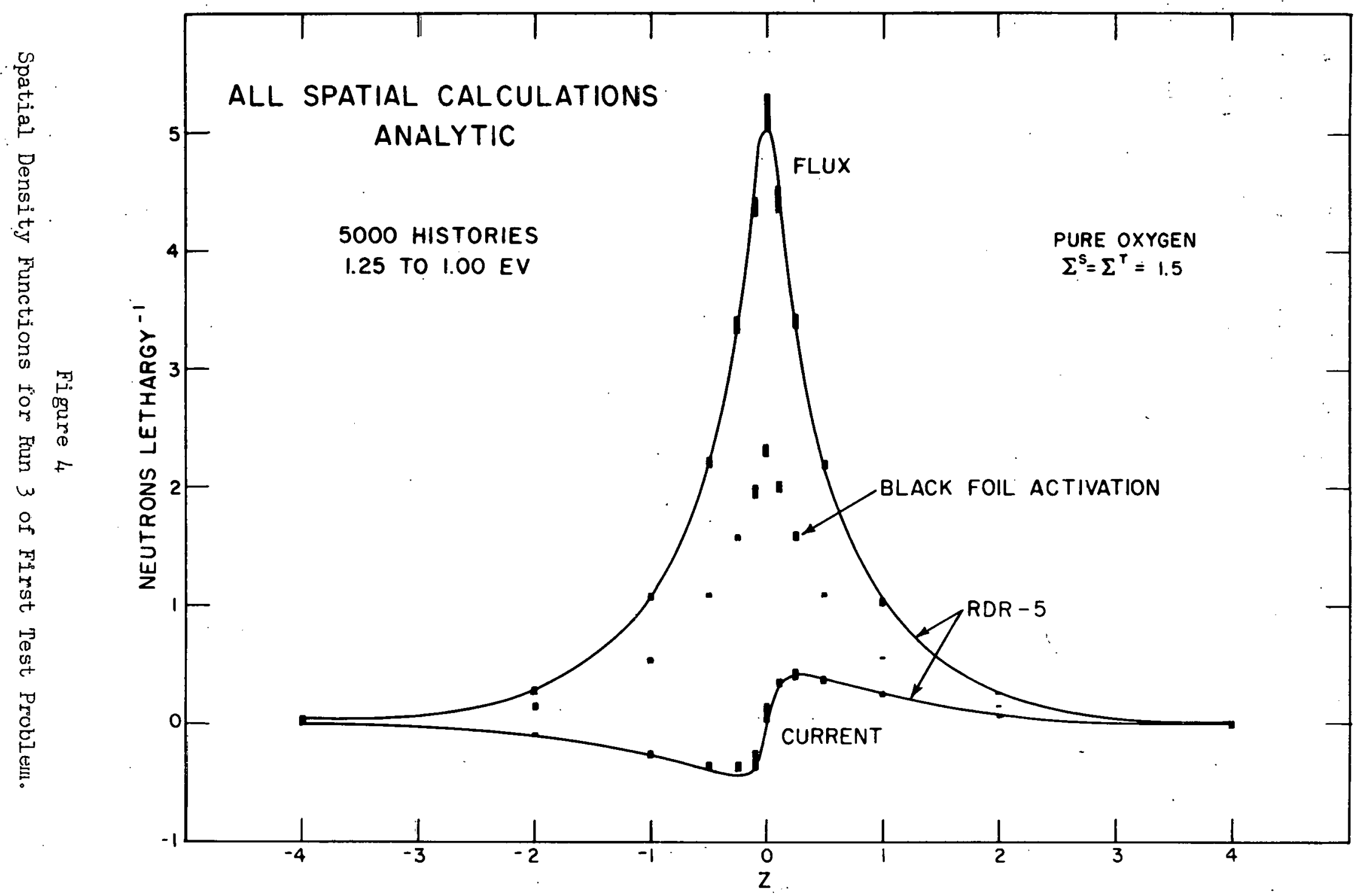


In Figso 2 through 4 the spatial density functions for the three runs are shown as solid bars that reach from the mean value plus the probable error to the mean value minus the probable error. Confusion as to which quantity a given bar refers may be avoided by noting that for each $z$ the value of the flux density is greater than the black foil activation density, which in turn is larger than the current density. The widths of the bars have no significance, there being no error in choosing $z_{0}$ The black foll activation at $z=1$ has been artificially displaced slightly in Fig. 3 to avoid overlap with the other functions. On each graph, two continuous lines show the flux and current densities calculated by $\mathrm{RDR}$ (Ref。2) with ten groups equally spaced in lethargy。

The three figures show about the same relative merit as their moments, but the property is also displajed that performing all spatial calculations analytically avoids the possibility of particular space points accidentally having much larger standard deviations than their neighbors. Since quite different techf... niques were used to calculate each of these three figures, the fact that they all agree to within their expected errors is strong evidence that each is correct。 III. The Second Sample Problem: Pure hydrogen with constant scattering cross section, zero absorption, isotropic monoenergetic source, $F\left(\mu_{R}\right)=\mu_{R}$ 。

For hydrogen, $\alpha=1$ in $E q_{0}(14)$, and the solution in Eqs. (15) through (28) not only tremendously simplify, but they are also valid at all energies below the source:

$$
\begin{aligned}
& k x_{00}=\left(\Sigma^{\mathrm{T}}\right)^{-1} \\
& k x_{11}=\left(\Sigma^{\mathrm{T}}\right)^{-2}\left[1-(1 / 3)\left(\mathrm{E}_{\mathrm{R}} / \mathrm{E}_{0}\right)^{1 / 2}\right] \\
& R x_{02}=2\left(\Sigma^{\mathrm{T}}\right)^{-3}\left[2 / 3+(1 / 3)\left(\mathrm{E}_{\mathrm{R}} / \mathrm{E}_{0}\right)^{1 / 2}+l\left(\mathrm{E}_{\mathrm{O}} / \mathrm{E}_{\mathrm{R}}\right)\right]
\end{aligned}
$$

Another interesting analytic property of this problem is that the average number of collisions in hydrogen is $1+\not h\left(E_{0} / E_{R}\right)$. Also, the slowing down density for 44. 
hydrogen equals the "slowing in source" of neutrons per unit lethargy". Since this problem has no absorption, the zeroth moment of the slowing down density for each history should be unity; furthermore, with no absorption, the zeroth moment of the slowing in source must equal the zeroth moment of the collision density. Therefore the zeroth moment of the flux should be rigorous each history, and no statistical error should ever result.

In the DAEDALUS test problems, $E_{R} / E_{0}=10^{-6}, \Sigma^{T}=1.4, \epsilon=4, \rho=R=0$ 。 Each experiment consisted of 500 histories. The first run had $N=0$ and went to six experiments; the second run set $N=50$ and went to twenty experiments。 Results follow。

\begin{tabular}{|c|c|c|c|c|}
\hline $\begin{array}{c}\text { Full Range } \\
\text { Moments of } \\
\text { Flux }\end{array}$ & $\begin{array}{l}\text { Analytic } \\
\text { Results }\end{array}$ & $\begin{array}{l}3000 \text { Histories } \\
\text { with } 1 \text { Analytic } \\
\text { Spatial Calc. }\end{array}$ & $\begin{array}{l}3000 \text { Histories } \\
\text { with up to } 51 \\
\text { Anal. Spat. Calcs }\end{array}$ & $\begin{array}{l}10_{8} 000 \text { Histories } \\
\text { with up to } 51 \\
\text { Anal. Spat。 Calcso }\end{array}$ \\
\hline 0 & 0.7143 & $0.7143 \pm 0.00004$ & $0.7143 \pm 0.00004$ & $0.7143 \pm 0.00004$ \\
\hline 1 & 0 & $-0.042 \pm 0.034$ & $0.026 \pm 0.032$ & $0.001 \pm 0.02$ \\
\hline 2 & 10.56 & \pm .0 .20 & \pm 0.17 & $10.56 . \pm 0.10$ \\
\hline 3 & 0 & \pm 2.4 & \pm 2.0 & $0.135 \pm 1.1$ \\
\hline
\end{tabular}

Full Range

Moments of Current

$\begin{array}{cccccc}0 & 0 & 0.0006 \pm 0.005 & 0.01 \pm 0.005 & 0.002 \pm 0.003 \\ 1 & 0.5100 & 0.516 \pm 0.019 & 0.488 \pm 0.018 & 0.50 \pm 0.01 \\ 2 & 0 & -0.125 \pm 0.145 & 0.0467 \pm 0.131 & 0.06 \pm 0.07\end{array}$

Average

Number of

Collisions:

14.82

$14.69 \pm 0.05$

$14.93 \pm 0.05$

$14.85 \pm 0.03$

For the zeroth moment of the $\mathrm{flux}_{8}$ the quoted error, being the same in all cases and being zero in all $(\epsilon=4)$ decimal places guaranteed free of round-off error, is undoubtedly completely due to round-off rather than statistical error. 


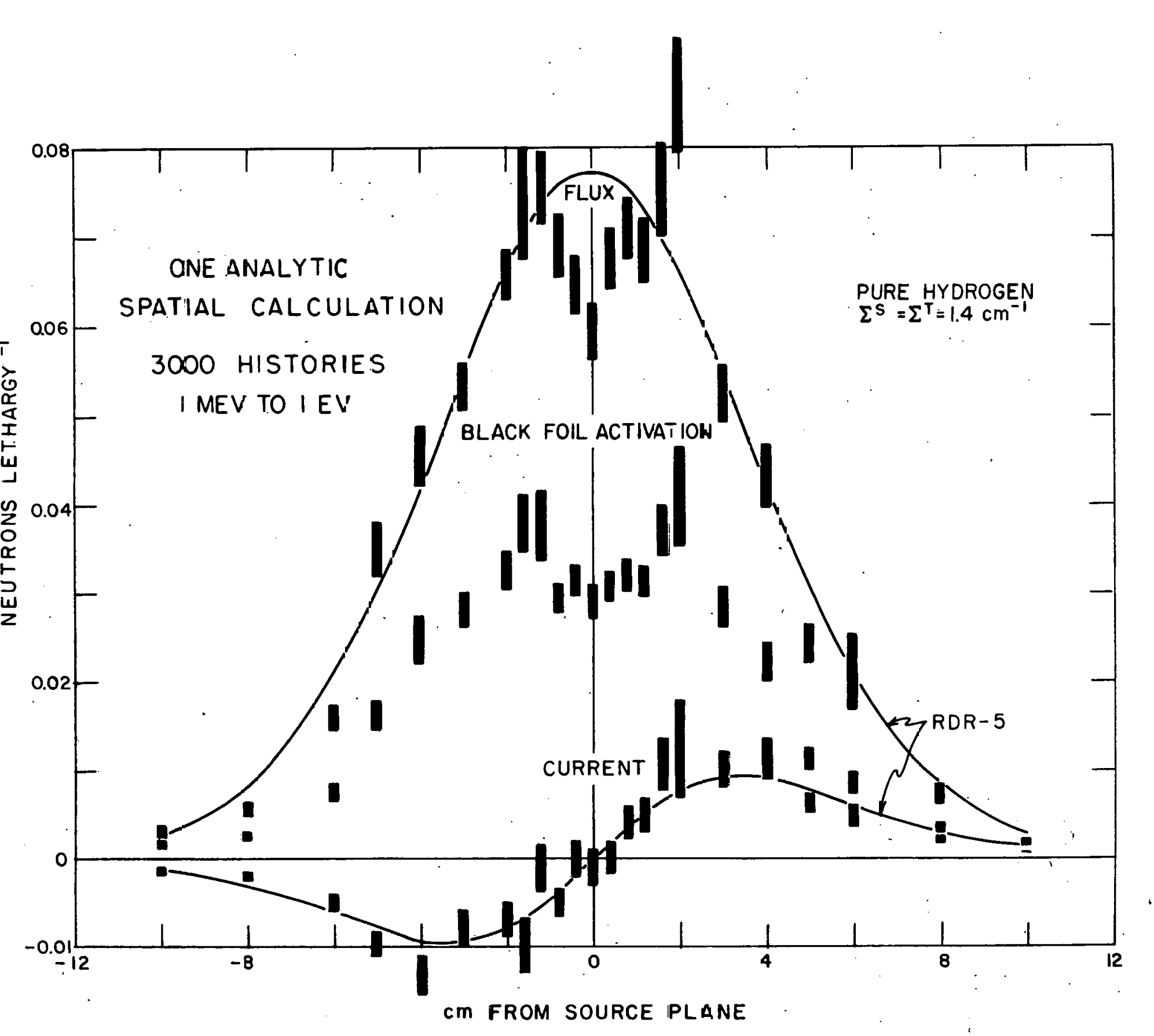



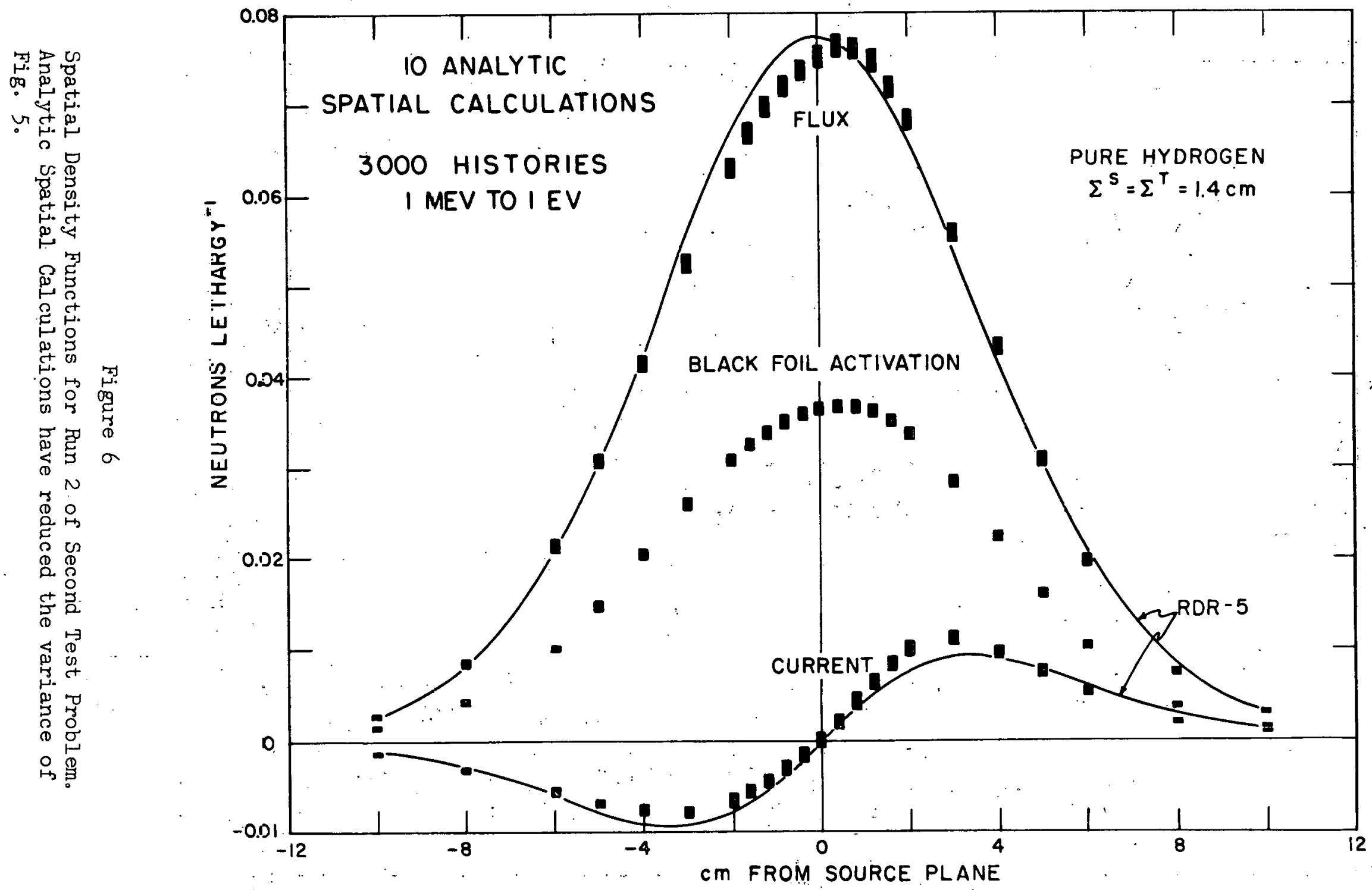


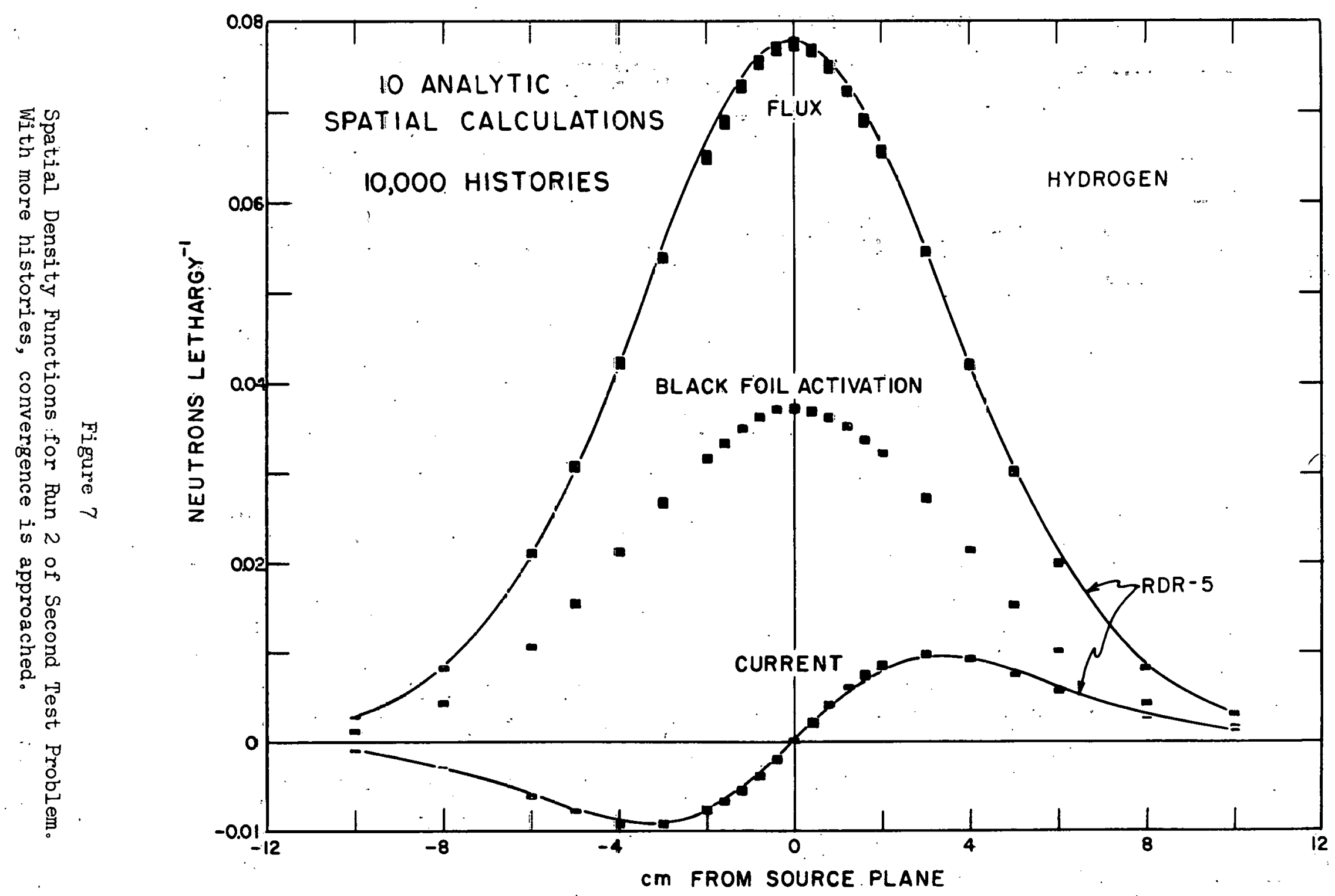


When $\mathrm{N}=50$ (last two columns in table), the average number of Monte Carlo position calculations was $5.61 \pm 0.036$ 。

It'is somewhat surprising that only a slight gain in the accuracy of the moments results when the spatial calculations are done analytically, especially in view of the fact that there is a tremendous improvement in the spatial density functions as displayed in Figs: 5 through 7, which have the same interpretation as Figs。 2 through 40 The continuous lines for the current and flux densities are from a 56 group RDR (Ref。2) calculation。

IV. The Third Somple Problem: EURIPUS-3 calculates slowing down density for the conditions of the second sample problem.

The moments. of the slowing down density for hydrogen can easily be obtained from those of the flux by integrating the latter over the slowing down kermel:

$$
v_{p q}=\int_{0}^{u}\left[1-e^{-\left(u-u^{0}\right)}\right] x_{p q}\left(u^{p}\right) d u^{0} .
$$

In partialar, the second moment of the slowing down density for this sample problem is

$$
(2 / 3)\left(\Sigma^{\mathrm{T}}\right)^{-2}\left[3 / \mathrm{n}\left(\mathrm{E}_{\mathrm{O}} / \mathrm{E}_{\mathrm{R}}\right)+2 \cdot \sqrt{\mathrm{E}_{\mathrm{R}} / \mathrm{E}_{0}}-1\right]
$$

The results of a 3000 history EURIPUS -3 problem with $N=50$ and other parameters the same as in the second sample problem gives a second moment equal to $13.85 \pm 0.24$ compared with an analytic answer of 13.76 . There were $14.88 \pm 0.05$ collisions on the average compared with an expectation value of 14082 . The average number of Monte Carlo position calculations equaled $6,17 \pm 0,06$.

Figure 8 displays the spatial density function along with realts of the same 56 group RDR calculation used for the second sample problem。 The quantity actually calculated by RDR was the slowing-in source, but as mentioned for the last problem, this quantity equals slowing down density for hydrogen。 

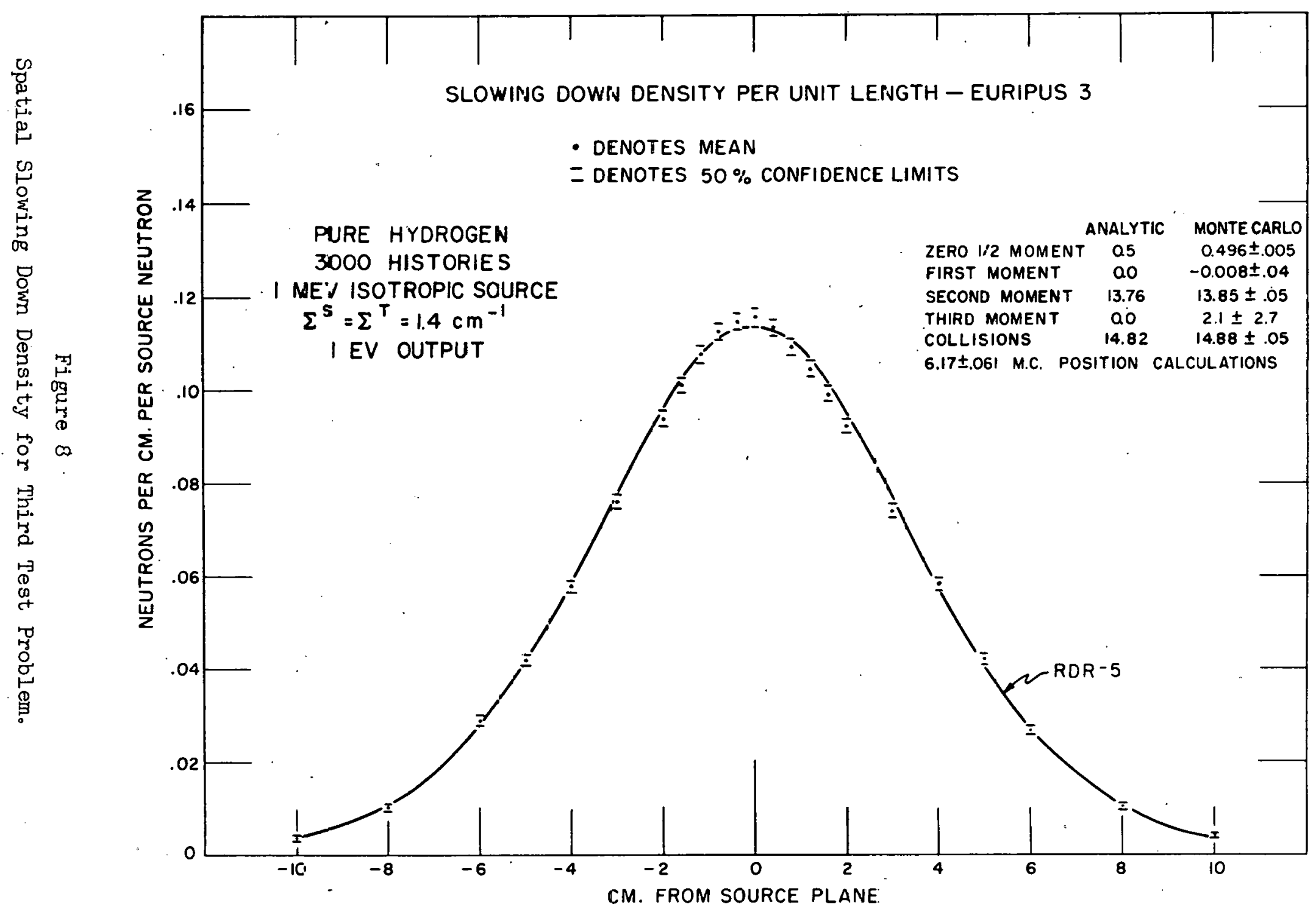
V. The Fourth Sample Problem: For DAEDALUS, pure hydrogen scattering with no absorption for an energy interval below the source energy; pure moderator A scattering with absorption for energies below this interval, cross sections constant in intervals, moderator A scattering isotropic in center of mass system, fraction of historles split; no source tube.

A problem was run with the following input:

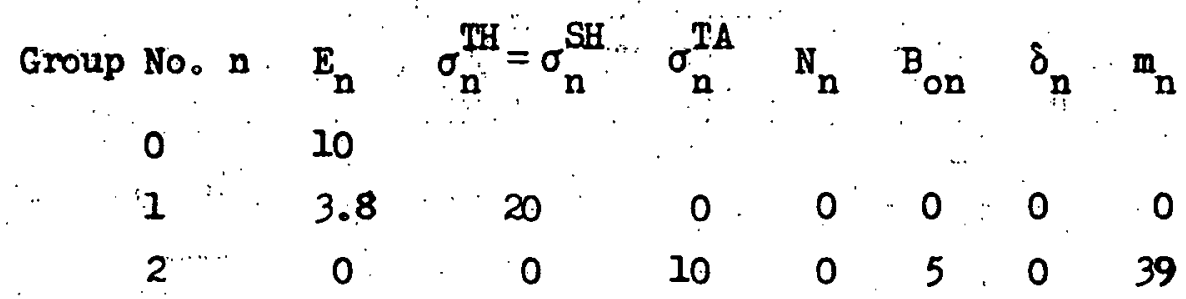

Isotropic source energy $E_{0}=6$

Hass No: $A=3$ implying $\alpha=0.25$

Splitting parameter $f=0.6$

Hydrogen No. denstto $\mathrm{N}_{\mathrm{H}}=0.3$

Max. Anal. Collistions- $1 \quad N=4$

Moderator $\mathrm{A} \mathrm{No}$ density $\mathrm{N}_{A}=0.2$

Decimal place accuracy $\epsilon=4$

Histories $\mathrm{N}_{\mathrm{H}}=2500$ :

Evaluation Energy $E_{R}=1$

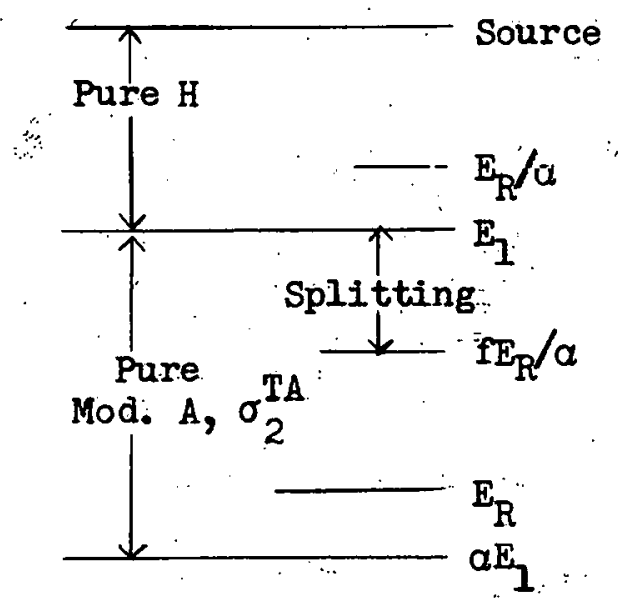

Positions of Energy Boundaries

Figure 9 
The analytic solution for the zeroth moment of the flux is

$$
\ell X_{O 0}\left(u_{R}\right)=E_{R^{\varphi_{2}}}\left(E_{R}\right)=\frac{1}{\Sigma_{2}^{T}}\left(\frac{E_{1}}{E_{R}}\right)^{\left(\frac{\Sigma_{2}^{S}}{\Sigma_{2}^{T}(1-\alpha)}-1\right)}=\frac{1}{2}(3.8)^{-1 / 3}=0.321
$$

DAEDALUS gives $\quad \ell X_{00}=.3204 I \pm 0.0020$

Since a neutron with energy above $E_{\eta}$ has an equal chance of going into any energy interval below, the fraction of neutrons whose first collision with moderator $A$ is above $\mathrm{fE}_{\mathrm{R}} / \alpha$ so that it will split is

$$
\left(E_{1}-f E_{R} / \alpha\right) / E_{1}=7 / 19
$$

In DAEDALUS, 933 histories split, compared to an expectation value of $2500(7 / 19)=921$.

VI. The Fifth Sample Problem: Hydrogen and moderator A both present, source monoenergetic and monodirectional, no absorption, constant cross sect1ons, 1sotropic in center of mass system, slight majority of histories split, no source tube.

A problem was run with the following input:

$$
\begin{aligned}
& \mu_{0}=1 ; E_{0}=3 \text { for the source } \\
& A=3, \text { so } \alpha=0.25 \\
& \Sigma^{T H}=\Sigma^{S H}=1 ; \Sigma^{S A}=\Sigma^{T A}=2 \\
& \varepsilon=5, N=3, f=0.5 \\
& E_{R}=1, N_{H}=2500 \text { histories. }
\end{aligned}
$$


The analytic answer for the zeroth moment of the flux is

$$
\chi X_{00}=\frac{K}{\Sigma^{S}}\left(\frac{E_{0}}{E_{R}}\right)^{K-1} \text { with } K \equiv \frac{\Sigma^{S}-\alpha \Sigma^{S H}}{(I-\alpha) \Sigma^{S}}
$$

We can thus compare the analytic answer $\ell X_{00}=0.5203$ with the calculated value; $.5229 \pm .0020 .1892$ histories were split.

VII. The Sixth Sample Problem. For EURIPUS-3, pure hydrogen with constant scatter ing and absorption cross sections, isotropic monoenergetic source.

The conditions of this problem are the same as those of the third sample problem except absorption is present: $E_{R} / E_{0}=10^{-6}, \Sigma^{S}=1.4, \Sigma^{T}=1.4739506$, $\varepsilon=4, \rho=l=0, \mathrm{~N}_{\mathrm{H}}=3000$ histories。

The analytic answer for the zeroth moment of the slowing down density is

$$
\frac{\Sigma^{\mathrm{S}}}{\Sigma^{\mathrm{T}}}\left(\frac{E_{\mathrm{R}}}{E_{0}}\right)^{l-\left(\Sigma^{\mathrm{S}} / \Sigma^{\mathrm{T}}\right)} \cdot=0.4749 ;,
$$

compared to the measured value,

$$
0.4759+0.0011
$$

Ais expected, when normalized to unit area, the graph of the slowing down density per unit length resembled that of Fig. 8, except that the absorption made the curve more peaked。 


\section{PART IV: A PROOF THAT DAEDALUS IS UNBIASED}

\section{Jerome Spanier}

In this section a proof is given that the basic random variable used by DAEDALUS provides unbiased estimates of the scalar flux $\Phi\left(z_{,} u_{R}\right)$ at lethargy $\mathrm{u}_{\mathrm{R}}$ and arbitrary prescribed spatial points. $\mathrm{z}_{0}$. The activation of a thin "black" foil and the flux-weighted integral of an arbitrary function of the final direction are easily obtained from the basic random variable. In addition, the low order full and half-range moments of these functions are estimated by an additional spatial integration of the basic random variables which estimate the functions.

The Monte Carlo code associates with each neutron history of energies and angles one number for each spatial coordinate, measuring the contribution of that history to the flux at the prescribed spatial point. This real-valued function on the space of histories is a random variable whose expected value will be identified with the Neumann series solution of the integral transport equation for the flux, evaluated at the space point in question. The expected values of the auxiliary random variables used in DAEDALUS are then easily identified with the appropriate weighted integrals of the flux. This is what is meant by the statement that DAEDALUS is unbiased.

In the analysis to follow details concerning the special techniques which are designed to prevent growth of round-off errors: and overflow problems are omitted despite the fact that these features are important in the utility

The code actually makes use of estimates of the vector flux $\varphi\left(z, \mu, u_{R}\right)$, where $\mu$ is a randomly selected final direction. However, these quantities do not appear in the final edit. 
of the code. An attempt to handle them explicitly would unduly complicate the analysis without adding anything essential. For this reason it was considered preferable merely to mention their effect on the theory briefly at the end of this section.

The problem, then, is to construct the proper estimator on historiesi of angles and energies under the assumption that unlimited accuracy is obtainable in the evaluation of all recursion formulas. In this respect the analysis is more pertinent to the test code EURIPUS-2 (see the discussion in Part I), but modified to apply to the flux rather than the slowing down density. The techniques used in this section are patterned after those of Ref。 1, where more complete detail and background material may be found.

It will be convenient to derive the integral transport equation for the vector collision density $\psi(z, \mu, E)$ rather than for the flux $\varphi(z, \mu, E)$. This equation has the form

$$
\psi(z, \mu, E)=\int_{0}^{\bar{E}} \int_{-1}^{1} \int_{\infty}^{\infty} \psi\left(z^{\gamma}, \mu^{\gamma}, E^{\gamma}\right) K\left(z, \mu, E_{\rho} z^{\gamma}, \mu^{\gamma}, E^{\gamma}\right) d z^{\gamma} d u^{\gamma} d E^{\gamma}+S(z, \mu, E),
$$

where $\mathrm{z}$ is the single space coordinate, $\mu$ is the cosine of the angle between the direction of the neutron and the $z$ axis, $E$ is the neutron energy, and $\bar{E}$ is the maximum source energy. In Eq. (I) the source term $S$ is the density of first collisions and is so normalized that its integral over phase space is unity。 The kernel $\mathrm{K}$ represents the probability density of transition from $\left(\mathrm{z}^{8}{ }_{2} \mu^{8}, \mathrm{E}^{\mathrm{V}}\right)$ to $(z, \mu, E)$ and is so normalized that

\footnotetext{
The collision density is the product of the flux and the total macroscopic cross section.

See, for example, Appendix 2 of Ref. 2 .
} 


$$
\int_{0}^{E^{p}} \int_{-1}^{1} \int_{-\infty}^{\infty} K\left(z, \mu, E ; z^{p}, \mu^{\gamma}, E^{\gamma}\right) d z d \mu d E=\frac{\Sigma^{S}\left(E^{p}\right)}{\Sigma^{T}\left(E^{p}\right)}
$$

where $\Sigma^{S}\left(E^{\prime}\right)$ and $\Sigma^{T}\left(E^{\gamma}\right)$ are the macroscopic scattering and total cross sections, at energy $E^{\eta}$. The function $K$ is factored into a product of two functions, one dealing only with energy and angular changes, and the other only with changes in position:

$$
K\left(z, \mu, E ; z^{p}, \mu^{p}, E^{p}\right)=C\left(\mu, E ; \mu^{\prime}, E^{p}\right) T\left(z ; z^{\prime}, \mu, E\right)
$$

where $T\left(z ; z^{i}, \mu, E\right)$ gives the density of next collisions at $z$ for a particle leaving a collision or source at $z^{\prime}$ with direction $\mu$ and energy $E_{9}$ and $C\left(\mu, E_{g} \mu^{\gamma}, E^{\prime}\right)$ gives the density of particles leaving a collision with direction $\mu$ and energy $\mathrm{E}$ after entering with direction $\mu^{\gamma}$ and energy $E^{\gamma}$. Explicitly, $T$ can be written

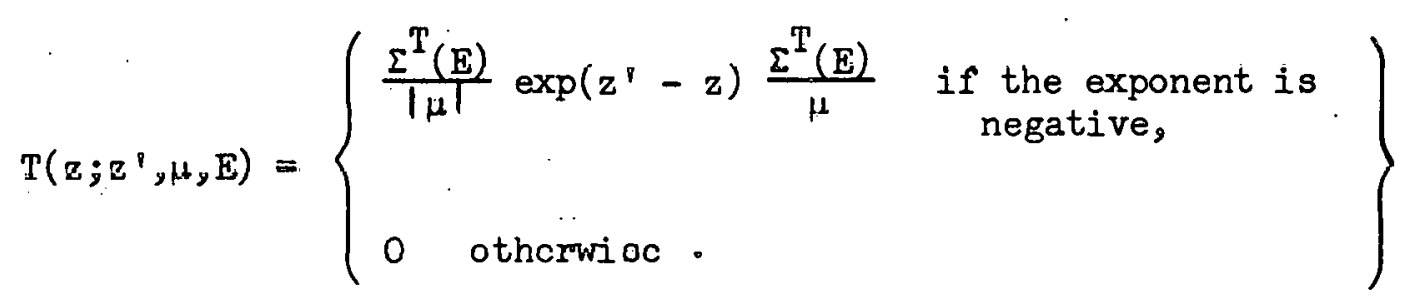

The scattering kernel $\mathrm{C}$ is somewhat more complicated to describe. The assumptions that hydrogen scattering is isotropic in the center of mass system and that the second isotope has arbitrary mass $A$ and arbitrary energy-dependent differential scattering cross sections leads to the form

$$
\begin{aligned}
& C\left(\mu, E ; \mu^{\prime}, E^{\prime}\right)=\frac{\Sigma^{S}\left(E^{1}\right)}{\Sigma^{T}\left(E^{\gamma}\right)}\left[\frac{\Sigma^{S H}\left(E^{\prime}\right)}{\Sigma^{S}\left(E^{\prime}\right)} \mid \frac{1}{E^{p}}\right) f_{H^{\prime}}\left(\mu ; \mu^{p}, E, E^{\gamma}\right) \\
& \left.+\frac{\Sigma^{S}\left(E^{\prime}\right)-\Sigma^{S H}\left(E^{\prime}\right)}{\Sigma^{S}\left(E^{\gamma}\right)} g_{A}\left(E ; E^{p}\right) f_{A}\left(\mu ; \mu^{p}, E_{,} E^{\gamma}\right)\right] \text {, }
\end{aligned}
$$


where $\Sigma^{S H}\left(E^{?}\right)$ is the macroscopic hydrogen scattering cross section at energy $E ?, f_{H}\left(\mu ; \mu^{\gamma}, E_{,} E^{\prime}\right)$ and $f_{A}\left(\mu^{\prime} \mu^{\gamma}, E_{,} E^{\prime}\right)$ are probability density functions for the new direction $\mu$ conditional upon the old direction $\mu^{8}$ and upon the selection of the energy $E$, and $g_{A}\left(E ; E^{\prime}\right)$ is the density of scattering into energy. $E$ with the second isotope. The density functions $f$ are not used explicitly by the code; the new direction $\mu$ is calculated by a random choice of a scattering angle (which determines.E), followed by the random selection of an azimuthal angle. The direction $\mu$ is then calculated by formula (see formula $D$ on page 21 of Part $I$ )。 The normalized probability density function defined by the scattering kernel is

$$
\begin{aligned}
C^{\#}\left(\mu, E ; \mu^{\gamma}, E^{\prime}\right) & =\frac{\Sigma^{T}\left(E^{\gamma}\right)}{\Sigma^{S}\left(E^{\gamma}\right)} C\left(\mu, E ; \mu^{\prime}, E^{\prime}\right) \\
& =C_{H}^{A}\left(\mu, E_{;} \mu^{\gamma}, E^{\prime}\right)+C_{A}^{*}\left(\mu, E ; \mu^{\prime}, E^{\prime}\right)
\end{aligned}
$$

where: $C_{H}^{A}$ is the density function for hydrogen scattering and $C_{A}$ is the corresponding function for scattering by isotope A.

The Neumann series for the collision density evaluated at the point $(z, \mu, E)$ is the infinite series of integrals.

$$
\begin{aligned}
& \psi(z, \mu, E)=S(z, \mu, E)+\int_{0}^{\frac{\pi}{E}} \int_{-1}^{T} \int_{-\infty}^{\infty} S\left(z_{0}, \mu_{0}, E_{0}\right) K\left(z_{1}, \mu_{1}, E_{1} ; z_{0}, \mu_{0}, E_{0}\right) d z_{0} d \mu_{0} d E_{0} \\
& +\int_{0}^{\mathrm{E}} \int_{-1}^{1} \int_{-\infty}^{\infty} \int_{0}^{\mathrm{E}} \int_{-1}^{1} \int_{-\infty}^{\infty} s\left(z_{0}, \mu_{0}, \mathrm{E}_{0}\right) K\left(z_{1}, \mu_{1}, \mathrm{E}_{1} ; \mathrm{z}_{0}, \mu_{0}, \mathrm{E}_{0}\right) \\
& K\left(z_{2}, \mu \mu_{2}, E_{2} ; z_{1}, \mu_{1}, E_{1}\right) d z_{0} d \mu_{0} d E_{0} d z_{1} d \mu_{1} d E_{1}+\cdots \cdots
\end{aligned}
$$


The $\mathrm{k}^{\text {th }}$ term of the series represents contributions to the collision density from neutrons which have collided $k-1$ times. The $(r+1)^{\text {st }}$ term of the series evaluated at energy $E_{\mathrm{R}^{2}}$

$$
\begin{gathered}
I_{r+1}\left(z_{2} \mu_{2} E_{R}\right)=\int_{0}^{\bar{E}} \cdots \int_{-\infty}^{\infty} S\left(z_{0}, \mu_{0}, E_{0}\right) \cdots \\
K\left(z_{1}, \mu_{1}, E_{7} ; z_{0}, \mu_{0}, E_{0}\right) \cdots K\left(z_{2}, \mu_{,} E_{R} ; z_{r-1}, \mu_{r-1}, E_{r-1}\right) d z_{0} \cdots d E_{r-1},
\end{gathered}
$$

will now be examined in greater detail.

First the assumption is made, as it is in the code, that for collisions which might slow a neutron to an energy below $E_{R^{\prime}}$ all scattering is isotropic in the center of mass system. This would imply for the isotope of mass A that

$$
g_{A^{i}}\left(E_{\xi} E^{\gamma}\right)=\left\{\begin{array}{ll}
\frac{1}{E^{\prime}-\alpha E^{\gamma}} & \text { if } \alpha E^{\prime} \leq E \leq E^{\gamma}, \\
0 & \text { if } E<\alpha E^{\gamma},
\end{array}\right\}
$$

where $\alpha=\left(\frac{A-1}{A+1}\right)^{2}$. It is now convenient to display the $(r+1)^{\text {st }}$ term of the Neumann series as a sum of two terms, one corresponding to collision with hydrogen, the other to collision with the second isotope. Thus,

$$
I_{r+1}\left(z, \mu, E_{R}\right)=I_{r+1}^{H}\left(z, \mu, E_{R}\right)+I_{r+1}^{A}\left(z, \mu, E_{R}\right)
$$

and, under the assumption that $E_{r} \leq E_{R^{2}}$ the term corresponding to hydrogen is 


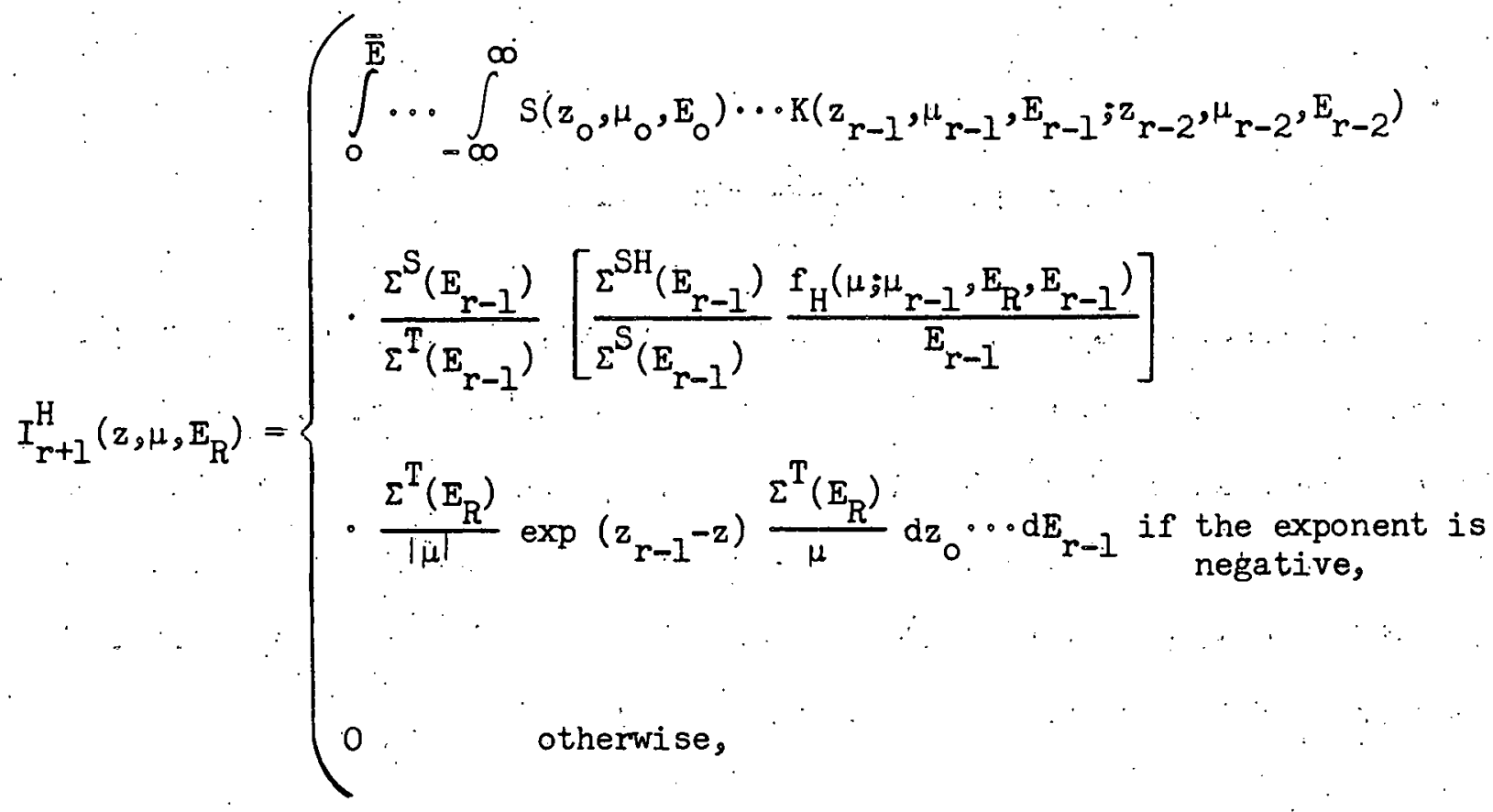

while the term corresponding to the second isotope is

$$
\begin{aligned}
& \int_{0}^{\overline{\mathrm{E}}} \cdots \int_{-\infty}^{\infty} S\left(z_{0}, \mu_{0}, \mathrm{E}_{0}\right) \cdots K\left(z_{r-1}, \mu_{r-1}, E_{r-1} ; z_{r-2}, \mu_{r-2}, E_{r-2}\right) \cdot \\
& \frac{\Sigma^{S}\left(E_{r-1}\right)}{\Sigma^{T}\left(E_{r-1}\right)}\left[\frac{\Sigma^{S A}\left(E_{r-1}\right)}{\Sigma^{S}\left(E_{r-1}\right)} \frac{f_{A}\left(\mu_{;} \mu_{r-1}, E_{R}, E_{r-1}\right)}{E_{r-1}-\alpha E_{r-1}}\right]
\end{aligned}
$$

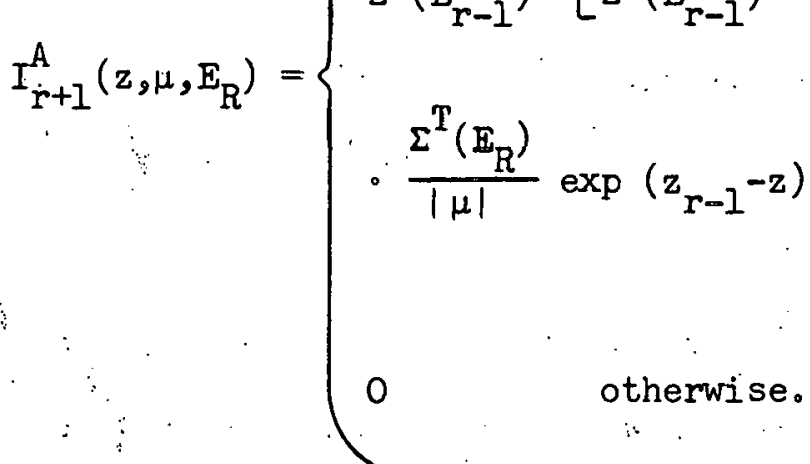

$$
\begin{aligned}
& \text { Now defining } \\
& Q\left(z ; z^{\gamma}, \mu, E\right)=\left\{\begin{array}{cc}
\frac{\Sigma^{S}(E)}{|\mu|} \exp \left(z^{1}-z\right) \frac{\Sigma^{T}(E)}{\mu} \text { if the exponent is negative, } \\
\quad \vdots \\
0 & \text { otherwlse, }
\end{array}\right.
\end{aligned}
$$


it follows that

$$
Q\left(z ; z^{\gamma}, \mu, E\right)=\frac{\Sigma^{S}(E)}{\Sigma^{T}(E)} T\left(z ; z^{\gamma}, \mu, E\right)
$$

where $\mathrm{T}$ is defined by Eq. (4). The function $Q\left(z ; z^{\prime}, \mu, E\right)$ is identical with the function $Q\left(z^{\prime}, z\right)$ defined by Eq. (1) of Part I; the notation is changed to display the dependences explicitly. Similarly, let $P_{i}\left(z ; \mu_{0}, E_{0}, \cdots, g \mu_{i}, E_{i}\right)$ be the probability density for an $(i+1)^{\text {st }}$ scattering collision at $z$ for particles furnished by the source at $z=0$ with direction $\mu_{n}$ and energy $E_{0}$ and with intermediate collision parameters $\mu_{1}, E_{1}, \cdots, \mu_{i}, E_{i}$. Then, as in Eq. (2) of Part I,

$$
P_{0}^{\prime}\left(z ; \mu_{0}, E_{0}\right)=Q\left(z ; O, \mu_{0}, E_{0}\right)
$$

and, as in Eq. (3) of Part $I_{9}$

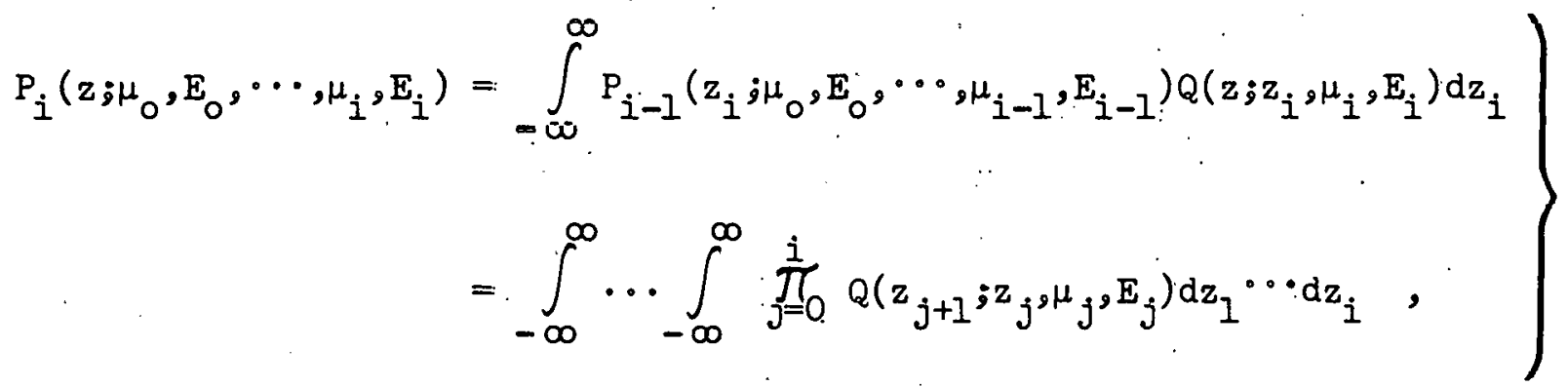

with the convention that $z_{0}=0, z_{i+1}=z$.

The two expressions (11) and (12) corresponding to hydrogen and the second isotope are now to be rewritten in such a way that the random variable used by DAEDALUS appears explicitly as part of the integrand, using. Eqs.' (14); (15), and (16). This will exhibit the relationship between the expected value of the random variable and the Neumann series. For the hydrogen term ( 11 ), using the fact that 


$$
S\left(z_{0} ; \mu_{0} ; E_{0}\right)=T\left(z_{0} ; O ; \mu_{0} ; E_{0}\right) S^{K}\left(\mu_{0}, E_{0}\right)
$$

where $S^{K}\left(\mu_{0}, E_{0}\right)$. is the probability density function for initial directions $\mu_{0}$ and initial energies $E_{o}$, one writes

$I_{r+1}^{H}\left(z, \mu, E_{R}\right)=\int_{0}^{\bar{E}} \cdots \int_{-\infty}^{\infty} S^{K}\left(\mu_{0}, E_{0}\right) T\left(z_{0}, 0, \mu_{0}, E_{0}\right) \frac{\Sigma^{S}\left(E_{0}\right)}{\Sigma^{T}\left(E_{0}\right)} \cdot c^{K}\left(\mu_{1}, E_{1}, j \mu_{0}, E_{0}\right) T\left(z_{1} ; z_{0}, \mu_{1}, E_{1}\right) \cdots$

$\cdot \frac{\Sigma^{S}\left(E_{r-2}\right)}{\Sigma^{T}\left(E_{r-2}\right)} c^{*}\left(\mu_{r-1}, E_{r-1} ; \mu_{r-2}, E_{r-2}\right) T\left(z_{r-1} ; z_{r-2}, \mu_{r-1}, E_{r-1}\right) \frac{\Sigma^{S}\left(E_{r-1}\right)}{\Sigma^{T}\left(E_{r-1}\right)}$

$\left[\frac{\Sigma^{S H}\left(E_{r-1}\right)}{\Sigma^{S}\left(E_{r-1}\right)} \frac{f_{H}\left(\mu_{q} \mu_{r-1}, E_{R}, E_{r-1}\right)}{E_{r-1}}\right] \cdot T\left(z_{;} z_{r-1}, \mu, E_{R}\right) d z_{0} \cdots d E_{r-1}$

where the order indicated in the integrand is the same as in the random walk in. phase space. By Eqs。(14) and (16)

$$
\begin{aligned}
& I_{r+1}^{H}\left(z, \mu, E_{R}\right)=\int_{0}^{\dot{\bar{E}}} \cdots \int_{-\infty}^{\infty} \cdot Q\left(z_{0} ; 0, \mu_{0}, E_{0}\right) \cdots Q\left(z_{r-1} ; z_{r-2}, \mu_{r-1}, E_{r-1}\right) Q\left(z_{j} ; z_{r-1}, \mu, E_{R}\right) \frac{\Sigma^{T}\left(E_{R}\right)}{\Sigma^{S}\left(E_{R}\right)} \\
& . S^{k}\left(\mu_{0}, E_{0}\right) c^{k}\left(\mu_{1}, E_{1} \beta \mu_{0}, E_{0}\right) \cdots c^{k}\left(\mu_{r-1}, E_{r-1} s \mu_{r-2}, E_{r-2}\right) c_{H}^{k}\left(\mu, E_{R} g \mu_{r-1}, E_{r-1}\right) d \varepsilon_{0} \cdots d E_{r-1} \\
& =\int_{0}^{\bar{E}} \cdots \int_{-1}^{I} P_{r}\left(z ; \mu_{0}, E_{0}, \cdots, \mu, E_{R}\right) \frac{\Sigma^{T}\left(E_{R}\right)}{\Sigma^{S}\left(E_{R}\right)} S^{k}\left(\mu_{0}, E_{0}\right) \cdots C_{H}^{k}\left(\mu, E_{R} ; \mu_{r-1}, E_{r-1}\right) d \mu_{0} \cdots d E_{r-1} .
\end{aligned}
$$

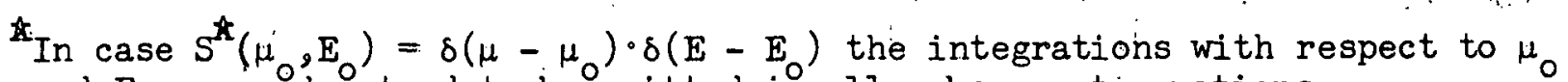
and $E_{0}$ are understood to be omitted in all subsequent equations. 
Notice that in the last integral all of the $z_{i}$ integrations have been performed explicitly. Finally, to reduce the integral to the precise form needed, it is

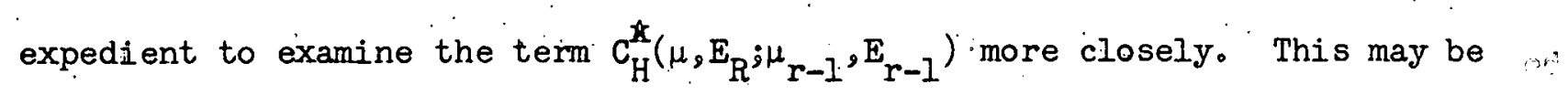
decomposed

$$
c_{H}^{K}\left(\mu, E_{R} ; \mu_{r-1}, E_{r-1}\right)=\left(\frac{\Sigma^{S H}\left(E_{r-1}\right)}{\Sigma^{S}\left(E_{r-1}\right)}\right) \cdot\left(\frac{E_{R}}{E_{r-1}}\right)\left(\frac{I}{E_{R}}\right) f_{H}\left(\mu ; \mu_{r-1}, E_{R}, E_{r-1}\right),
$$

the first factor representing the probability of scattering by hydrogen at energy. $E_{r-1}$, the second factor the probability that the energy drops from $\mathbb{E}_{\mathbf{r}-1}$ to a value below $E_{R^{\prime}}$ the third factor the conditional probability (density) of attaining energy $E_{R}$ provided the new energy is not greater than $E_{R}$, and the fourth factor the conditional probability density function for the direction $\mu$ given $\mu_{r-1}$, $E_{R^{\prime}}$ and $E_{r-I^{\circ}}$ An exactly similar analysis leads to the form

$$
\begin{gathered}
I_{r+1}^{A}\left(z_{2} \mu, E_{R}\right)=\int_{0}^{\bar{E}} \cdots \int_{-1}^{1} P_{r}\left(z ; \mu_{0}, E_{0}, \cdots, \mu, E_{R}\right) \cdot \frac{\Sigma_{:}^{T}\left(E_{R}\right)}{\Sigma^{S}\left(E_{R}\right)} \\
\cdot S^{\star}\left(\mu_{0}, E_{0}\right) \cdots C_{A}^{A}\left(\mu, E_{R} ; \mu_{r-1}, E_{r-1}\right) d \mu_{0} \cdots d E_{r-1}
\end{gathered}
$$

and a decomposition of $C_{A}^{\star k}\left(\mu, E_{R} ; \mu_{r-1}, E_{r-1}\right)$ as

$$
C_{A}^{A}\left(\mu, E_{R} ; \mu_{r-1}, E_{r-1}\right)=\left(\frac{\Sigma^{S A}\left(E_{r-1}\right)}{\Sigma^{S}\left(E_{r-1}\right)}\right)\left(\frac{E_{R}-\alpha E_{r-1}}{E_{r-1}-\alpha E_{r-1}}\right)\left(\frac{1}{E_{R}-\alpha E_{r-1}}\right) f_{A}\left(\mu ; \mu_{r-1}, E_{R}, E_{r-1}\right),
$$

with the same interpretation for the four factors. 
The Monte Carlo portion of DAEDALUS selects a source direction and energy $\mu_{0}, E_{0}$ from the density function $S^{k^{*}}\left(\mu_{0}, E_{0}\right)$ and then successive pairs $\mu_{i}, E_{i}$ from the densities $C^{k}\left(\mu_{i}, E_{i} ; \mu_{i-1}, E_{i-1}\right)$ until a specific collision results in an energy $E_{r}$ below $E_{R^{\circ}}$ At this point a final direction $\mu_{R}$ is chosen from either $f_{H}\left(\mu_{;} \mu_{r-1}, E_{R}, E_{r-1}\right)$ or $f_{A}\left(\mu_{;} \mu_{r-1}, E_{R^{2}} E_{r-1}\right)$ and the function

$$
\xi_{F}\left(z, \mu_{R}, E_{R}, \mu_{0}, E_{0}, \cdots, \mu_{r-1}, E_{r-1}\right)=\frac{P_{r}\left(z ; \mu_{0}, E_{O}, \cdots, \mu_{R}, E_{R}\right)}{\Sigma S_{R}\left(E_{R}\right)\left(E_{R}-\alpha E_{r-1}\right)}
$$

is evaluated, where $\alpha=0$ if the last collision is with hydrogen and otherwise $\alpha=\left(\frac{A-I}{A+I}\right)^{2}$. The formula (23) is to be used if flux per unit energy is desired. If flux per unit lethargy is needed, then the relation $d u_{R}=\frac{d E_{R}}{E_{R}}$ changes (23) to

$$
\xi_{F}\left(z_{,} \mu_{R}, u_{R}, \mu_{0}, E_{0}, \cdots, \mu_{r-1}, E_{r-1}\right)=\frac{P_{r}\left(z ; \mu_{0}, E_{0}, \cdots, \mu_{R}, E_{R}\right)}{\Sigma S\left(E_{R}\right)\left(1-\alpha \frac{E_{r}-1}{E_{R}}\right)}
$$

and the second factors of (20) and (22) are similarly altered. This function is the basic random variable of DAEDALUS, evaluated on sequences of directions and energies involving $r$ collisions before the energy falls below $E_{R^{\circ}}$ Now for each chain $C$ consisting of pairs of directions and energies $\mu_{i}, E_{i}$, $C=\left(\mu_{0}, E_{0}, \mu_{1}, E_{1}, \cdots, \mu_{r-1}, E_{r-1}, \cdots\right)$, define $n(C)$ to be the smallest integer such that $E_{n(C)} \leq E_{R^{\circ}}$. The expected value of the random variable defined by (23) or (24). is defined to be

$$
\mathrm{E}\left[\xi_{\mathrm{F}}\right]=\int_{\Omega} \xi(\mathrm{C}) \mathrm{dP}(\mathrm{C}),
$$

where $\Omega$ is the space of all possible chains $C$ and $\operatorname{dP}(C)$ denotes a specific probability measure on $\Omega$. A detailed discussion of these points may be found 
in Ref。 1, \$ 7. The expected value may be decomposed

$$
E\left[\xi_{F}\right]=\sum_{r=1}^{\infty} P\{n(C)=r\} E\left[\xi_{F} \mid n(C)=r\right],
$$

where $P\{n(c)=r\}$ is the probability that $n(C)=r$ and $E\left[\xi_{F} \ln (c)=r\right]$ is the conditional expected value of $\xi_{F}$ given that $n(C)=r$. From the definition of the measure $\mathrm{dP}(\mathrm{c})$ (see Ref。 $1, \$ 8, \mathrm{pp} .40-42$ for a similar argument) one can. write

$$
E\left[\xi_{F} \ln (C)=r\right]=\frac{1}{P\{n(C)=r\}}(A+B)
$$

where

$$
\begin{aligned}
& A=\int_{-1}^{1}\left[\int_{0}^{\bar{E}} \cdots \int_{-1}^{1} \xi_{F}\left(z, \mu_{R}, E_{R} ; \mu_{o}, E_{0}, \cdots, \mu_{r-1}, E_{r-1}\right)\right.
\end{aligned}
$$

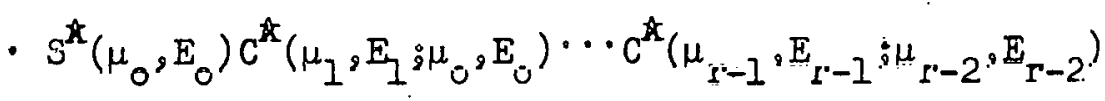

$$
\begin{aligned}
& \left.\cdot \frac{\Sigma^{S H}\left(E_{r-1}\right)}{\Sigma_{i}^{S}\left(E_{r-1}\right)} \cdot \frac{E_{R}}{E_{r-1}} \cdot f_{I I}\left(\mu_{R} ; \mu_{r-1}, E_{R}, E_{r-1}\right) d \mu_{j} \cdots d E_{r-1}\right] d \mu_{R}
\end{aligned}
$$

and

* The assumption is made in DAEDALUS that the minimum source energy is larger than $\mathrm{E}_{\mathrm{R}}$; hence $\mathrm{P}\{\mathrm{n}(\mathrm{C})=0\}=0$. 


$$
\begin{aligned}
& B=\int_{-1}^{1}\left[\int_{0}^{\mathrm{E}} \cdots \int_{-1}^{1} \xi_{F}\left(z ; \mu_{R^{2}}, E_{R} ; \mu_{0}, E_{0} ; \cdots, \mu_{r-1}, E_{r-1}\right)\right. \\
& \text { - } S^{\star}\left(\mu_{0}, E_{0}\right) C^{\star}\left(\mu_{1}, E_{1} ; \mu_{0}, E_{0}\right) \cdots C^{k}\left(\mu_{r-1}, E_{r-1} ; \mu_{r-2}, E_{r-2}\right) \\
& \left.\therefore \frac{\Sigma^{S A}\left(E_{r-1}\right)}{\Sigma^{S}\left(E_{r-1}\right)} \cdot \frac{E_{R}-\alpha E_{r-1}}{E_{r-1}-\alpha E_{r-1}} f_{A}\left(\mu_{R} ; \mu_{r-1}, E_{R}, E_{r-1}\right) d \mu_{\circ} \cdots d E_{r-1}\right] d \mu_{R} \text {. }
\end{aligned}
$$

Inserting the definition (23) of $\xi_{F}$ and comparing (28) and (29) with (19) and (2I), one finds that

$$
\Sigma^{T}\left(E_{R}\right) A=\int_{-1}^{1} I_{r+1}^{H}\left(z, \mu, E_{R}\right) d \mu
$$

and

$$
\Sigma^{T}\left(E_{R}\right) B=\int_{-1}^{1} I_{r+1}^{A}\left(z, \mu, E_{R}\right) d \mu
$$

Then

$$
\Sigma^{T}\left(E_{R}\right) \cdot B\left[\xi_{F} \ln (C)-1 \cdot-\frac{1}{P\{n(C)=r\}} \cdot \int_{-1}^{I} I_{r+1}\left(\alpha, \mu, E_{R}\right) d \mu\right.
$$

The integral on the right is the integral with respect to $\mu$ of the $(r+1)^{\text {st }}$ term of the Neumann series for the vector collision density (Eq: (7)) . Assuming the convergence of the series in question, which assumptions may be justified at least heuristically, one is led to 


$$
\begin{aligned}
& \Sigma^{T}\left(E_{R}\right) E\left[\dot{\xi}_{F}\right]=\sum_{r=1}^{\infty} \int_{-1}^{I} I_{r+1}\left(z, \mu, E_{R}\right) d \mu \\
& =\int_{-1}^{1} \psi\left(z, \mu, E_{R}\right) d \mu \\
& =\Psi\left(\mathrm{z}, \mathrm{E}_{\mathrm{R}}\right) \text {, }
\end{aligned}
$$

the scalar collision density. This establishes that

$$
E\left[\xi_{F}\right]=\Phi\left(z, E_{R}\right)
$$

the scalar flux, and completes the proof of the unbiased character of $\xi_{F^{\circ}}$ Now if $M(\mu)$ is any function, define a random variable $\xi_{M}$ by

$$
\xi_{M}\left(z_{2} \mu_{R}, E_{R}, \mu_{0}, E_{0}, \cdots, \mu_{r-1}, E_{r-1}\right)=M\left(\mu_{R}\right) \xi_{F}\left(z_{,} \mu_{R}, E_{R} ; \mu_{0}, E_{0}, \cdots, \mu_{r-1}, E_{r-1}\right)
$$

and the same analysis will show that

$$
\dot{E}\left[\xi_{M}\right]=\int_{-1}^{I} M(\mu) \varphi\left(z, \mu, E_{R}\right) d \mu
$$

so that $s_{M}$ yields an unbiased estimate of the integral on the right. In particular the choice $M(\mu)=|\mu|$ is automatically made by the code to yield the activation of a thin "black" foil and provision is made to input a table of values for an arbitrary $M(\mu)$, linear interpolation being used to evaluate $M\left(\mu_{R}\right)$. Finally, the low order momento of $\Phi\left(\mathrm{z}_{j} \mathrm{E}_{\mathrm{R}}\right)$ arc eotimated by explicitly

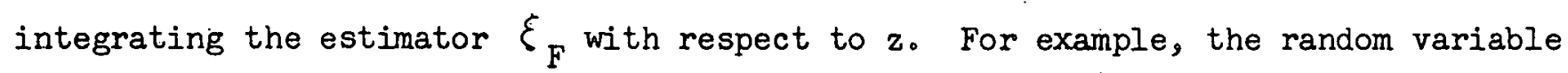




$$
\tau_{F}^{(k)}=\int_{-\infty}^{\infty} z^{k} \xi_{F} \mathrm{dz}
$$

has as its expected value the integral

$$
t_{F}^{(k)}=\int_{-\infty}^{\infty} z^{k} \Phi\left(z, E_{R}\right) d z \quad
$$

The same method is used to estimate moments of the other functions calculated by DAEDALUS。

As mentioned in the beginning of this section, the foregoing analysis has not taken into account the special techniques which DAEDALUS uses to avoid growth of round-off errors and overflow. For completeness these will now be discussed briefly.

As was mentioned in Part I, round-off errors may occur in evaluating the estimator $\xi_{F}$ by using Eq. (4) of Part I. For this reason, DAEDALUS discontinues the use of this formula after round-off is detected and calculates the last spatial positions by random choices: The estimator that actually is used by the code will then be a function of these spatial positions $z_{i}$. Its expected value, however, will be identical with the expected value of the $\xi_{F}$ defined in Eq. (23) since all of the intermediate positions $z_{i}$ are integrated out in taking the expected value. As a matter of fact, it is not even essential that the Monte Carlo positions be chosen at the end of the history; they can occur anywhere in the chain. The resulting estimator will simply omit the integrations corresponding to these points and will still have the correct expected value. Thus the round-off criterion does not introduce any bias. 
Furthermore, the splitting technique discussed in Part I is also unbiased for this merely splits off part of the history which would normally contribute to the $(r+1)^{\text {st }}$ term of the Neumann series and forces it to contribute to a later term in the series. As long as the correct weight is carried along with this segment of the history no bias is introduced.

\section{References}

1. J. Spanier, "Monte Carlo Methods and Their Application to Neutron Transport Problems," WAPD-195 (July 1959)。

2. G。 Goertzel and M. H. Kalos, "Monte Carlo Methods in Transport Problems," Progress in Nuclear Energy, Series I, Physics and Mathematics, Vol.2, D. H. Hughes, Jo E。 Sanders, and Jo Horowitz, eds, Pergamon Press (1958). 


\title{
PART V.O. CODE OPERATION
}

\author{
Heidi G. Kuehn
}

\section{INTRODUCTION}

The DAEDALUS and EURIPUS-3 codes are written for an IBM-704 computer: equipped with a $32 \mathrm{~K}$ memory and, in addition to the minimum standard equipment, five tape units and an IBM-7I7 (off-line) printer. Except where differences are specifically indicated, the following discussion applies equally well to both the DAEDALUS and EURIPUS-3 codes。

DAEDA:LUS and EURIPUS bo.th consist of three parts - the Main Portion, the Source Preparation Routine, and the Cross Section Library Routine. Included in the Main Portion are the input, the history generating (Part. I, Section VIII), the statistical, and the output routines. For the purpose of restarting a problem, the Main Portion records on tape 4 the statistical data accumulated from the history generating routine after every experiment, as well as pertinent input parameters of the problem. The Main Portion coordinates the three parts of the code and checks for consistency in input when starting or restarting a problem.

The Source and Cross Section Routines, described in Sections VI and VII of Part Is may be entered only at the beginning of a now problom. The data generated by these routines are stored in memory and on tapes 2 and 3, respeco tively, from where they are obtained by the Main Portion throughout the remainder of the problem。

\section{INPUT}

For initiating or restarting a problem, the input data for the three parts of DAEDALUS and EURIPUS are read either from cards or from both cards and tape. At the beginning of a new problem, the entire input deck of the Main 
Portion is prepared; input information which is necessary for a restart of the same problem is then written on tape 40 When a problem is restarted, the Main Portion input deck consists of only the title card, the "ID" card, and - for DAEDALUS only - possible "z deletion" cards; the remaining input and accumulated statistical data are obtained from the restart tape (tape 4) of the particular problem。

For the Source Preparation Routine, a control word (ID2) in the Main Portion input indicates whether the neutron source is monoenergetic, from the $D \times D$ (deuteron-deuteron) reaction (the energy table fon which is generated onto tape 2) or from the $D-D$ reaction where the data are read from a previously generated source tape (tape 2)。 When the source is monoenergetic, (1) tape 2 is not used (2) the source input deck immediately follows that of the Main Portion when the problem is initiated, and (3) no source input is necessary upon restarting. If a problem requires new D-D source data, the energy table is developed and is stored on tape 2 before the history generating routine is initiated. In this case the source input deck immediately follows that of the Main Portion at the start of the problem; when the problem is restarted, no source input deck is needed, but the appropriate tape 2 containing the source data must be mounted. Similarly, if the $D \circ D$ source for a particular problem is the same as that for another problem, the previously generated tape 2 may be used when starting and restarting the new problem, and no source input deck is necessary.

A second control word (ID3) in the Main Portion input determines whether the Cross Section Library Routine will be entered to produce new differential cross section data or whether the cross section data stored on tape 3 by another problem can be used. In the former case, if a new cross section tape (tape 3 ) is prepared before entering the history generating routine of a problem the input deck for this routine follows that of the source or that of the Main Portion, depending upon whether a source input deck is required. For a restart of the 
problem, the library of cross section data is then obtained entirely from tape 3. When the cross section data is the same as for another problem, the previously generated tape:3 is read when starting and restarting the new problem, and the cross section inpü deck is not needed."

The card input for the Main Portion, the Source Preparation Routine, and the Cross Section Library Routine is discussed below in detail。 After the input for all three parts has been read, the program tests for consistency in the energy groups: The energy at output, $E_{R^{\prime}}$ must be less than the minimum possible source energy; the highest energy group in the cross section library, $E_{0}$, must be greater than the maximum possible source energy: and the lowest energy group in the cross section library, $E_{K^{\prime}}$ must be equal to or less than the energy at output, $\mathbf{E}_{\mathrm{R}^{\circ}}$

Ali input cards for DAEDALUS and EURIPUS-3, except the title card, are read into the computer by the WHOOI subroutine and therefore must be punched according to WHOOL specifications (SHARE distribution \# 57)。 The DAEDALUS input sheets (see Appendix) and the following write-up indicate whether the input values (words) are represented as fixed-point ( $f$ ) or floating-point (F) numbers; the input sheets also show the length of each word. Two additional blank cards should always be placed at the end of the entire input deck regardless of whether this deck initiates or restarts a problem.

\section{MAIN PORTION}

Title Card: This card should contain information identifying the problem. This information may be punched. in any format desired in columns 1-72. The title card is printed at the beginning of each page of the on-line and off-line output.

ID Card: The first nine values on this card are fixed-point integers; the tenth, $f$, is a floating-point word. Since $f$ is required only in DAEDALUS, columns 53-72 of the EURIPUS-3 ID card must be punched with zeros. 
PROBID: the number used to identify the problem in print out as well as for restart identification. (Note: At Bettis, the problem number given on thé tally card should equal PROBID in order to facilitate tape reference.)

$\mathrm{N}_{\mathrm{H}}: \quad$ the number of histories per experiment to be mun $\mathrm{N}_{\mathrm{H}}>1$. $\mathrm{J}:$ the experiment number with which the run is to begin. For an initial run, $J$ equals one. When a problem is restarted, $J$ is one plus the number of experiments which have previously been run for the problem.

$\mathbb{N}_{E}:$ the number of the last experiment which is to be run uniess an ending is forced.

$\mathrm{N}$ : one less than the maximum number of analytic position calculations per history. $0 \leq \mathrm{N} \leq 100$.

ع.: the number of decimal places of accuracy in the evaluation of spatially dependent output quantitico. $0 \leq \varepsilon \leq 35$.

ID2: the source identification. If a D-D energy table is to be generated on logical tape 2, ID2 must equal PROBID. If the source is monoenergetic (and either isotropic or monodirectional), ID2 must equal zero. If a previously generated source tape can be used, ID2 equals the PROBID of the run which generated that specific source tape (tape 2)。

ID3: the library cross section tape (tape 3) identification. If a new tape is required, ID3 must equal PROBID. If a previously generated cross section tape is used, ID3 equals the PROBID which was used in the generation of the cross section tape. 
REC: a D-D source table occupies one record on the source tape (tape 2). REC specifies the number of the record to be read from or written onto this tape. $R E C=1$ unless the results of several $D-D$ table generations are stored consecutively on the same logical tape 2. If the source is monoenergetic, tape. 2 is not used and REC equals zero.

f: the splitting parameter (section VIII. (8), part I).

Parameter Card: Of the six parameters listed, the last three are specified only in DAEDALUS. The parameter card for EURIPUS-3 must therefore have zeros in columns 20-72. The values for $M_{2}, P$, and $M_{T}$ are in fixed-point, and the remaining are in floating-point.

M: the number of spatial positions at which the z-dependent quantities are evaluated. $I \leq M \leq 100$.

$\mathrm{P}: \quad$ the hijghest order moment to be evaluated. $0 \leq P \leq 10$.

$E_{R}$ : the energy at which output quantities are evaluated. $E_{R}>0$ 。

$l:$ the distance from the point source to the end of its containing cylinder。

$\rho:$ the distance from the point source to the sides of its containing cylinder.

$\mathrm{M}_{\mathrm{T}}: \quad$ the number of table values for $\mathrm{F}\left(\mu_{\dot{\mathrm{R}}}\right) \cdot .2 \leq \mathrm{M}_{\mathrm{T}} \leq 50$ 。

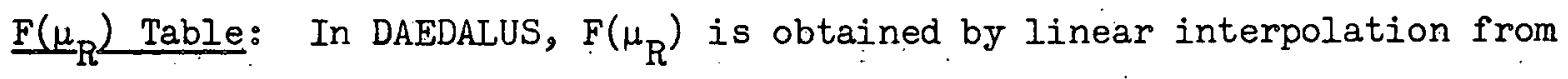
tabulated values of $F\left(\mu_{R}\right)$ al equal increments of $\mu_{R}$ from $\mu_{R}=-1$ to $\mu_{R}=+I_{0}$ Each of the $\left[\frac{M_{T}+7}{8}\right]$ input cards describing $F\left(\mu_{R}\right)$ contains. eight floating-point values, except the last card which may have less than eight numbers. The table gives consecutive values of $F\left(\mu_{R}\right)$ in 
increments of $2 / M_{T}-1$ from $\mu_{R}=-1$. to $\mu_{R}=+1$, inclusive. A blank card jos must follow the last card of the table.

The $F\left(\mu_{R}\right)$ table and blank card are not included in the EURIPUS-3 input deck。

Coordinate Cards: Two numbers appear on each card, the first being a fixed . point integer and the second a normalized floating point number。 One card must be in the deck for each $z$ input coordinate. The code requires that the origin (source plane) be one of the z. values. The M conritnate cards must be arranged in increasing order of $\mathrm{z}$ values, from minimum to maximum the code tests this monotonicity, and the program will stop if this test fails. A blank card follows the coordinate card of the maximum. $z$ value. OPT: option for on-line (in addition to off-line) output of statistical data at the $\mathrm{z}$ coordinate indicated on this card. $\mathrm{A}$ to is used If only an off-line print out is desired; $a+l$ is used if both an on-line and off-line print out is desired.

2: distarice from the source plane at which z-dependent tunctions are evaluated.

After the input for the Main Portion is read, the title card, PROBID $\mathrm{N}_{\mathrm{H}}$, $N, \varepsilon$, the parameter card values, and the minimum and maximum $z$ values are printed on the first page of on-line and off-line output.

\section{SOURCE PREPARATION}

\section{1,2. Monoenergetic Source}

In the case of a monoenergetic source, the angular diatribution of the source neutrons may be either isotropic or monodirectional. The input for this source consists of one card containing two normalized floating point numbers. 


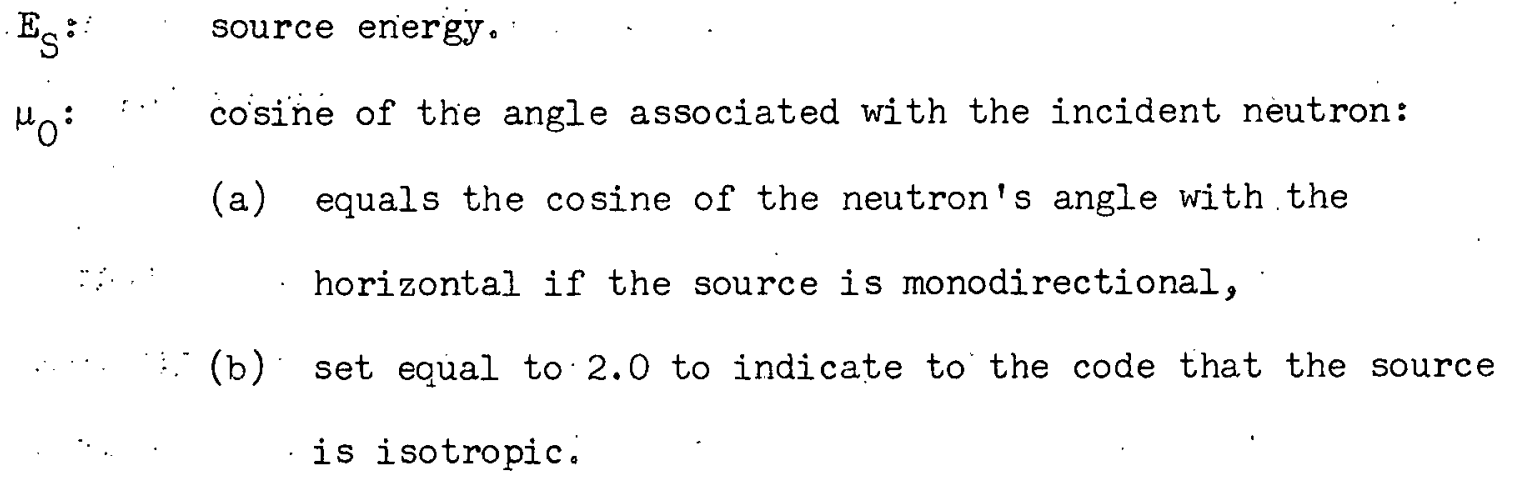

A blank card must follow this input card.

When the monoenergetic source has been read, the code prints the above information on-line and off-line after the input print out for the Main Portion.

\section{D-D Source}

The Source Preparation Routine generates onto logical tape 2 one record of information for each new D-D source. The records generated by various problems may be collected on one tape and must be numbered consecutively. The number of records that may be written on the same tape is limited only by the length of the tape. An option is provided in the Main Portion permitting utilization of a specified previously generated record and omission of the normal Source Preparation Routine for a D-D source. In either case, the record to be read or that to be written is adequately identified by the record number (REC) and problem numbers (PREID and ID2) specified on input cards.

Identification-Data Card: This, the first in the source input deck, contains seven numbers (only the last two of which are floating-point words) and inusl be pursched in the order described.

REC: number of the record to be generated on tape 2. This integer is the same as that for REC in the Main Portion input. PREID: problem number of the last generated record on tape 2 . If $\mathrm{REC}=1$, then a new tape 2 is being used and FREID is necessarily equal to zero. 
ID2: number of the current problem which will generate a new record on tape 2. This number must be identical to PROBID and ID2 in the Main Portion input。

$\mathrm{N}: \quad$ an integer $<16$. There are $\mathrm{N}+1$ coefficients for the Legendre polynomial expansion used to generate the angle-energy table.

$\mathrm{m}$ : an integer, $1 \leq \mathrm{m}<50$. There are $\mathrm{m}+1$ entries in the angle-energy table.

Q: the $Q$ of reaction.

$E_{D}: \quad$ deuteron energy. $E_{D} \neq 0$.

Coefficient Cards: These, the second through the $\mathrm{N}+2$ nd source input cards, contain the $\mathrm{N}+1$ coefficients, denoted by $S_{L}, L=0,1, \ldots, N$, for the Legendre polynomial expansion used to generate the angle-energy table for the source. Each of the $\mathrm{N}+1$ cards contains one normalized floating point coefficient. The $\mathrm{N}+\mathrm{l}$ coefficients must be designated even if they are zero. The cards of $S_{L}$ values are arranged consecutively as $L$ goes from 0 .to $N$; a blank card immediately follows the last coefficient card.

When the source tape has been generated or read, the values ID2, $Q$, $E_{D} ;$ and the Legendre coefficients are included with the input print out from the Main Portion at the beginning of a new problem.

\section{CROSS SECTION LIBRARY}

The Cross Section Library Routine generates onto logical tape 3 data used by the Main Portion of DAEDALUS and EURIPUS. The routine generates a variable number of records which may be described in this manner:

Record 1: a combination of constant data pertaining to the Cross Section Library Routine as a whole and blocks of data pero taining to each energy group. 
The remaining records: a maximum of 8 records, each containing a maximum of 50 angle-energy tables, $i_{.} e_{\circ}$, one table per energy group; the total number of energy groups is $K \leq 400$.

Data from only one problem may be written on a single tape. An option is provided in the Main Portion permitting utilization of a previously generated tape 3 and omission of the normal Cross Section Library Routine. If a previously generated tape is selected, the problem number written thereon is checked with ID 3 on the ID card of the Main Portion input deck to assure proper identification.

Identification-Constant Data Card: This, the first in the cross section input deck, contains six parameters; the first two are fixed-point words and the remaining are in floating-point.

ID3: number of the current problem which will generate tape 3. ID3 must correspond with PROBID and ID3 of the Main Portion input. $\mathrm{K}: \quad$ an integer $\leq 400$. Number of energy groups.

A: $\quad$ mass of moderator A.

$\mathrm{N}^{\mathrm{H}}$ : hydrogen number density.

$\mathrm{N}^{\mathrm{A}}$ : number density of moderator $A$.

$\mathrm{E}_{\mathrm{O}}: \quad$ maximum energy。

Energy Group Data Cards: Each of the $K$ energy groups may have a minimum of one card, a maximum of 3 cards, the use of cards 2 and 3 depending upon the size of each $N_{n}$ as described hereafter. Each of the constants is specified for $n=1, \ldots, K$.

Input energies, $E_{n}$, distinguishing end points of successive energy groups, decrease in magnitude from the first to the $K^{\text {th }}$. group. The Energy Group Data cards are inserted into the cross section library 
deck immediately following the Identification-Constant Data card ordered from the first to the $K^{\text {th }}$ energy groups. The data for each energy group, including the last, must be followed by a blank card.

Card 1 of each energy group contains a minimum of 8 numbers, a maximum of 12 and is punched in the following format:

$E_{n}: \quad$ the lowest energy of the $n^{\text {th }}$ energy group.

$\mathrm{N}_{\mathrm{n}}: \quad$ an integer $<2 l_{0}$. There are $\mathrm{N}_{\mathrm{n}}+\mathrm{l}$ coefficients for the Legendre polynomial oxpansion used to generate the differential cross section table for the $n^{\text {th }}$ energy group.

$m_{n}: \quad$ an integer, $1 \leq m_{n}<40$. There are $m_{n}+1$ mesh points in the differential cross section table.

$\delta_{n}: \quad \delta_{n}=0$ or 1 for every $n$.

$\delta_{n}=0$ if the differential cross sections of moderator A are in the C.M. system.

$\delta_{n}=I$ if the differential cross sections of moderator A are in the lab syetcm.

$\sigma_{n}^{T A}: \quad$ total microscopic cross section for moderator A.

$\sigma_{\mathrm{n}}^{\mathrm{TH}}$ : total microscopic cross section for hydrogen.

$\sigma_{n}^{\mathrm{SH}}$ : total microscopic scattering cross section for hydrogen.

$\mathrm{B}_{\mathrm{O}, \mathrm{n}}$ : the $\mathrm{N}_{\mathrm{n}}+1$ coefficients for the Legendre polynomial expansion, $:$

$\mathrm{B}_{4, \mathrm{n}}: \quad$ designated as $\mathrm{B}_{\mathrm{L}, \mathrm{n}}, \mathrm{L}=0,1, \ldots, \mathrm{N}_{\mathrm{n}} \cdot \mathrm{B}_{\mathrm{O}, \mathrm{n}} \neq 0$.

If $\mathrm{N}_{\mathrm{n}}>4$, card 2 of each energy group contains a maximum of ten $B_{\mathrm{L}, \mathrm{n}}$ values, $B_{5, n}, \ldots, B_{14, n}$, punched in the previously described $B_{L, n}$ form。 If $\mathrm{N}_{n}=14$, card 3 of each energy group contains a maximum of six $B_{i, n}$ values, $\mathrm{B}_{15, \mathrm{n}}, \ldots, \mathrm{B}_{\mathrm{N}_{\mathrm{n}}, \mathrm{n}}$, punched in the $\mathrm{B}_{\mathrm{L}, \mathrm{n}}$ form described above. 
The $\mathrm{N}_{\mathrm{n}}+\mathrm{I} \mathrm{B}_{\mathrm{L}, \mathrm{n}}$ values must be designated, even if they are zero。 Therefore, at least one $B_{L, n}$ value must always be designated, $i_{\circ} e_{\circ}$, one value when $N_{n}=0$. If the specified $B_{L, n}$ values do not completely fill any one of the cards for the energy group, the remaining words on the card should be zeros punched in the prescribed format.

When the cross section library tape has been generated or read, the values ID3, $K, A_{9} \mathrm{~N}^{\mathrm{H}}, \mathrm{N}^{\mathrm{A}}$, and the energy range from $\mathrm{E}_{\mathrm{K}}$ to $\mathrm{E}_{\mathrm{O}}$ are added to the input print out of the Main Portion and Source at the beginning of a new problem.

\section{RESTART}

For the restart of a problem, the cross section and source data are obtained entirely from tape 3 and tape 2 , if a D D source is specified, the same tapes as were used for the initial run of the problem. The problem restart tape (tape 4), containing the accumulated statistical data and problem parameters, is also required since it provides a significant part of the Main Portion restart input.

The only required card input for restarting DAEDALUS and EURIPUS -3 is the title card and ID card of the Main Portion. The values for PROBID, ID2, ID3, and REC must be the same on the restart ID card as when the problem was initiated. It should be noted that only the parameters $\mathrm{N}_{\mathrm{H}}, \mathrm{N}, \varepsilon$, and $f$ on the ID card may be changed within a given problem at the time of a restart.

An option provided in DAEDALUS makes it possible to delete the calculation of the flux, the foil activation, and the "extra. function" at specified $z$ points. This option is of particular value when sufficient accuracy in functional values is obtained much earlier (i.e., after fewer experiments) at certain spatial points than at others. By deleting these spatial points, the running time is reduced in subsequent experiments. 


\section{Deleted $z$ Values}

With the exception of the source plane coordinate, $z=0$, which is always required, as many $\mathrm{z}$ coordinates may be deleted as desired. The coordinates must be representied on the input cards of deleted $z$ values exactly as they were on the coordinate cards. If a discrepancy occurs or a non-existing coordinate value is specified, the program will stop after printing IS NOT RECOGNIZED AS A CURRENT $Z$ VALUE。 REMOVE PROBLEM. The corresponding print option, OPT, for the deleted coordinate will be removed by the program; thus there will be no on-line or off-line output for lhe deleted coordinate.

Each card, except the last, specifying deleted $\mathrm{z}$ values must consist of six floating point coordinate values. The last card may have less than six values in which case the remaining values on the card are set equal to zero. Ailthough the coordinate cards of the Main Portion must be arranged in ascending order, the non-zero values on the $\mathrm{z}$ deletion cards may be in any desired order. The $\mathrm{z}$ deletion cards go immediately after the restart ID card and are followed by a blank card. If no $\mathrm{z}$ coordinates are to be deleted when restarting a DAEDALUS problem, a blank card must be placed after the restart ID card.

After the restart input is read and the problem and tape identifications are successfully tested, the title card, the values PROBID, $\mathrm{N}_{\mathrm{H}^{\circ}} \mathrm{N}_{\text {, }} \varepsilon$, $f$ and RESTART INITIATED CORRECTLY are printed on the first page of on-line and offoline output. III. NITPIJT:

The DAEDALUS and EURIPUS output is from the IBM-716 (on-line) printer and from tape 5 (off-line). As the inpul fur each of the three parte of DAEDALUS and EURIPUS is read, the significant parameters are printed both on-line and. off-line to identify the total output of the problem more clearly. Should an input error be detected by one of the input routines, a brief explanation is 
printed on-line, the paper is restored, and the program stops. Similarly, when a problem is restarted, RESTART INITIATED CORRECTLY is printed if the tapes and input identification agree; if a discrepancy exists; however, it will be described in the print out, and the program will stop.

After each experiment of $\mathrm{N}_{\mathrm{H}}$ histories, the output on the printer and from tape consists of:

(1) the experiment number;

(2) the total number of histories generated;

(3) the total number of split histories. (DAEDALUS only);:

(4) the mean and confidence interval at the 0.50 level of the number of Monte: Carlo position calculations and collisions (in EURIPUS-3, the average number of collisions minus one is evaluated):

(5) for DAEDALUS, the mean and 0.50 confidence interval for the full- and half-moments of the flux, the black foil activation, and the "extra function" - a product of $F\left(\mu_{R}\right)$ and the flux where $F\left(\mu_{R}\right)$ is determined from an input table; for EURIPUS-3, the mean and 0.50 confidence interval for the full-and half-moments of the density function $q(z)$. The $\mathrm{P}^{\text {th }}$ moment is the highest order moment evaluated in both $\operatorname{codes}{ }^{*}$

(6) for DAEDALUS, the mean and 0.50 confidence interval of the flux, the black foil activation, and $F\left(\mu_{R}\right)$ times the flux at specified $z$ pointso for EURIPUS, the density function $q(z)$ at the $z$ points

The half-moments are written off-line only。 
and the corresponding 0.50 confidence intervals. These data are written on-line for only those points which are specified for on-line output.

The above statistics are based upon the accumulated data from all histories, beginning with the first experiment. After the completion of the last experiment $\left(\mathrm{N}_{\mathrm{E}}\right)$ or after an ending is forced, additional statistics are printed in the same format as above for those histories generated during the current machine run。 If a problem is initiated, these statistics are the same as those for the last experiment as described above. However, if a problem is restarted, these statistics are a function of the historles generated only in the restart run。

IV. COMPUTER OPERATING INSTRUCTIONS

A. Writing a program tape

The binary program deck of DAEDALUS and EURIPUS-3 consists of the binary deck for the Main Portion, the Source Preparation Routine, the Cross Section Library Routine, and WB CTB2 (which generates a one file binary tape from non-relocatable binary cards), combined with the appropriate control and sentinel cards. The routine WB CTB2 provides a tape loader, WB TSB?, stored as the first record on tape.

To write a program tape:

1. Mount a blank on tape 1 .

2. Place the binary program deck in the card hopper.

3. Press "load cards" on the 704 console.

4. End of program stop is at location $(0343) 8^{\circ}$

5. Error stops for WB CTB2 and WB TSB2 are listed in SHARE distribution \# 425 . 
.1. B Bunning a DAEDALUS or EURIPUS-3 problem

\section{Loading Instructions:}

1. Press "clear" on the 704 console.

2. Insert a GLOUT2 board into the 716 printer; "ready" the printer and the card punch.

3. Place the problem input deck in the card hopper and "ready" the card reader。

4. Adjust sense switch 1 according to the conditions described below. A:Il other sense switches are up.

5. Mount the program tape on tape $I$ and a blank tape for output on tape 5 .

6. The problem specifier must indicate whether no tape, a blank tape, or a specified tape is to be mounted on tape 2 for the source data; similarly he must indicate whether a blank or particular tape is to be mounted on tape 3 for the cross section library data. In the case of a restart, either tape 2 is not used or it contains the same tape as at the start of the problem; tape 3 always has the same tape as when the problem was initiated.

7. At the beginning of a new problem, tape 4 is blank. This tape is the restart tape for the problem and must always be mounted on tape unit 4 when muning the problem. 
8. Press "load tape". on the 704 console to start the program.

ch

Sense Switches:

Only sense switch $I$ is used for running the code. Sense switch $I$ is up except when a forced ending is desired. After sense switch 1 is put down, the program will stop when the experiment then running is completed and the output pertaining to this experiment has been written.

\section{End of Program:}

When the problem is completed or after a forced ending with sense switch 1, the computer stops on an HTR instruction with the location counter reading $74526 \mathrm{~g}$ for DAEDALUS and $11435_{8}$ for EURIPUS-3. The 704 console will be li't?:

All tapes except tape 5 are rewound by the program and should be saved, unless otherwise specified.

When the problem stops after the on-line print out of at least one experiment, tape 5 should be printed on the 717 printer with the carriage control set to "program".

\section{Program Error Stops:}

The error stops in the three parts of the DAEDALUS and EURIPUS -3 codes are listed in this section. The input routines have numerous checks 
for possible input errors, in which cases the type of error is printed on-line and the program stops. Subroutine and overflow error stops are also described by an on-line printout; the location of subroutine errors may be further identified by the address lights of the accumulator on the 704 console。

\section{Location}

DAEDALUS

$(00141)_{8}$

$(00175)_{8} \cdot(00175)_{8}$

$(00736)_{8} \quad(00732)_{8}$

$(01651)_{8} \quad(01645)_{8}$

$(01704)_{8} \quad(01700)_{8}$

$(01767)_{8} \cdot(01763)_{8}$

$(02202)_{8} \quad(02176)_{8}$

$(04317)_{8} \quad(04317)_{8}$

$(05257)_{8} \quad(0525.7)_{8}$

$(10327)_{8} \quad(07074)_{8}$
Explanation

UACSB2: Information card check sum verification error. The error bit(s) appear in the accumulator. Press start to continue loading.

UACSB2: The loader has encountered a relocatable card. There is no restart from this stop.

WBRWT5: End of tape test. Load appropriate tape and press "start" on 704 console to continue problem.

WHOOL: Copied card image does not match card image computed from $B C D$.

WHOOl: Original $B C D$ does not match BCD reconverted from binary.

WHOOl: Double punch, blank column.

WHOOl: Range error. Floating decimal number out of arange 。

For the above WHOOI stops, make the necessary corrections and restart problem.

General program-error stop in Source Preparation Routine. Check the on-line print out for further identification. Remove problem。

General program-error stop in Cross Section Library Routine. Check the on-line print out for further identification. Remove problem.

FDH on $\Sigma^{S} / \mu^{i}$ calculation。 Manually transfer to $(10222) \mathrm{g}$ in DAEDALUS or (07021) 8 in EURIPUS-3 to start a new history and continue with problem. 

$(10332)_{8} \quad\left(0^{\prime} 70^{\prime} 7\right)_{8} \quad$ FDH on $\Sigma^{T} / \mu^{i}$ calculation. Manually transter to $(10222)_{8}$ in DAEDALUS or $(07021)_{8}$ in EURIPUS- 3 to start a new history and continue with problem.

$(74653)_{8} \quad(11566)_{8} \quad$ General program-error stop in Main Portion... Check the on-line print out for further identification. Remove problem. 
DAEDALUS Input Sheets

A. Main Portion

B. Source Preparation Routine

C. Library Cross Section Routine

The expression ( $f$ ) after a symbol means that the symbol should be specified in fixed decimal notation.

The expression (F) after a symbol means that the symbol should be specified in floating decimal notation. 
TITLE CARD

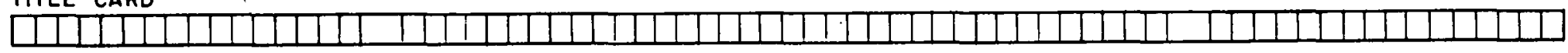
IO CARD

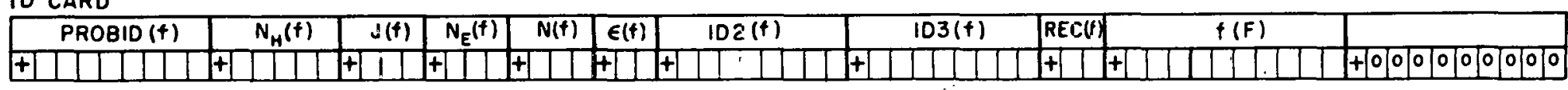

RESTART ONLY - DELETED \& VALUES (F)

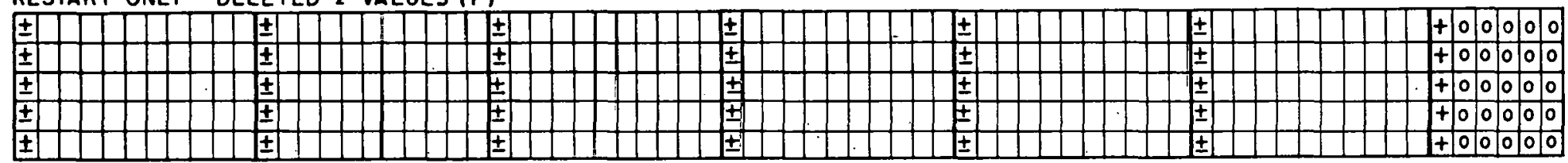

BLANK CARD AFTER LAST DELETION CARD

PARAMETER CARD

\begin{tabular}{|c|c|c|c|c|c|}
\hline$M(f)$ & $P(f)$ & $E_{R}(F)$ & $\rho(F)$ & $\rho(F)$ & $M_{T}(f)$ \\
\hline
\end{tabular}

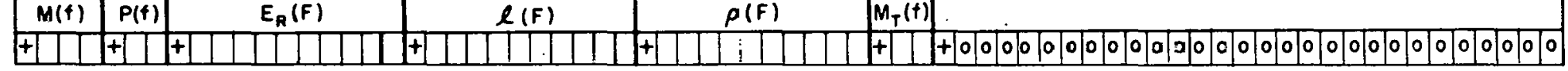

$F\left(\mu_{R}\right)$ TABLE $(F)$

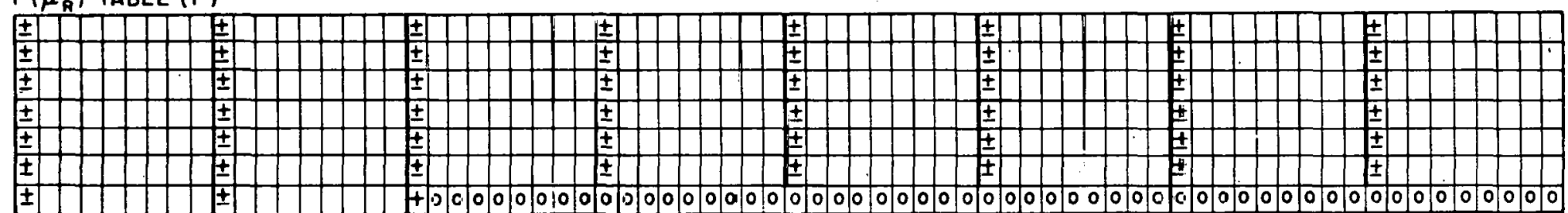

BLANK CARD

COORDINATE CARDS

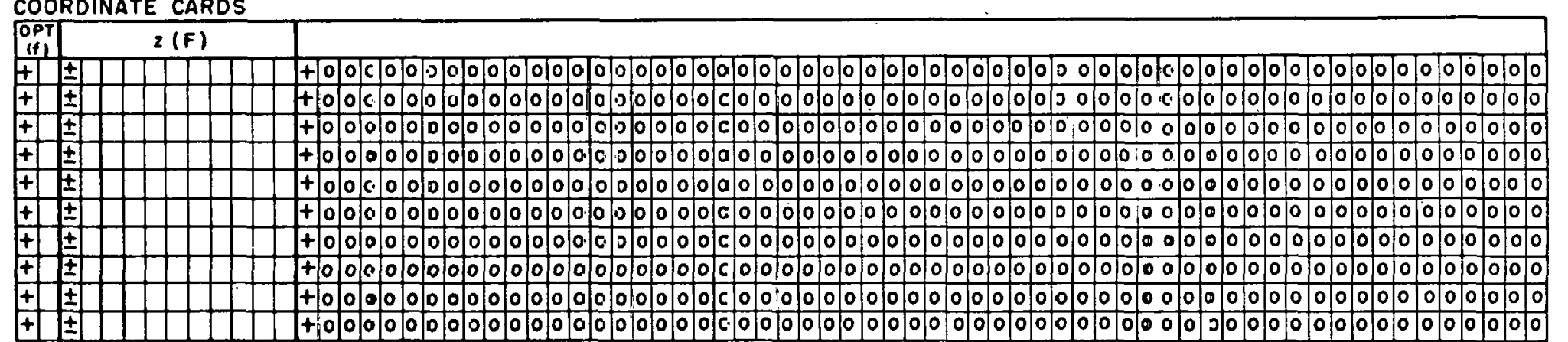

BLANK CARD AFTER LAST COQRDINATE CARO 
1. MONOENERGETIC - MONODIRECTIONAL SOURCE

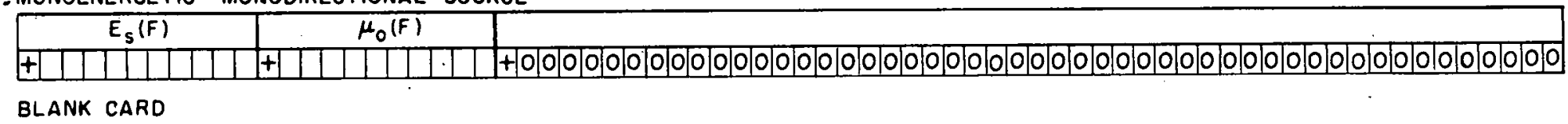

\section{MONOENERGETIC-ISOTROPIC SOURCE}

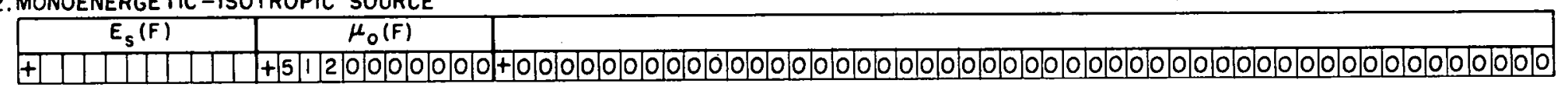

\section{BLANK CARD}

3. D-D SOURCE

Identificotion-Doto Card

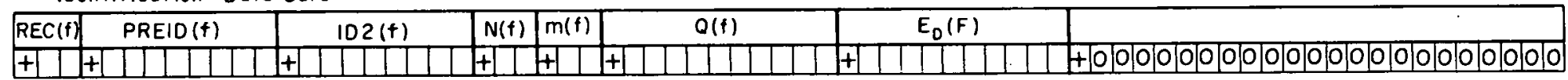

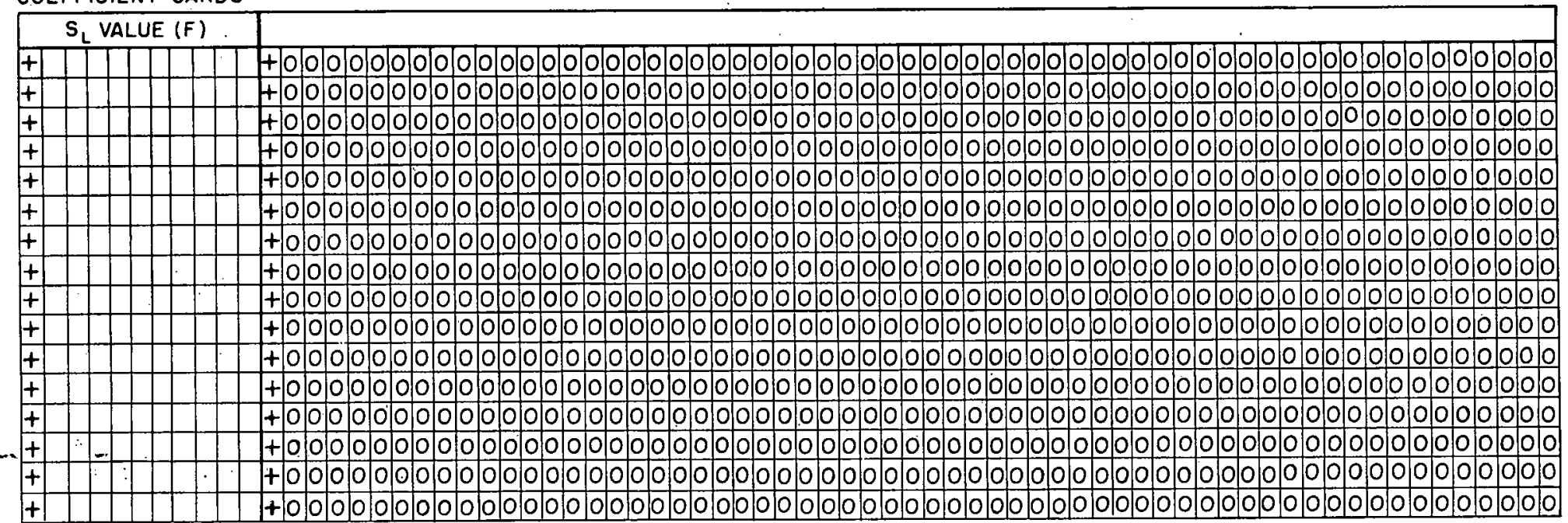

$$
\begin{array}{lll}
\ddots & \because & \ddots \\
\because & \ddots & \ddots
\end{array}
$$


IDENTIFICATION - CONSTANT DATA CARO

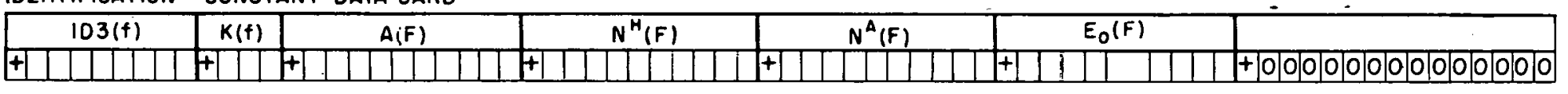

ENERGY GROUP GATA CARDS

Group

\begin{tabular}{|c|c|c|c|c|c|c|c|c|c|c|c|c|c|c|c|c|c|c|c|c|c|c|}
\hline & $E_{n}(F)$ & & $\mathrm{N}_{r}(\mathrm{t})$ & $n_{n}(t$ & 可 $\varepsilon_{n}(f)$ & & $\sigma_{n}^{T A}(F)$ & & $\sigma_{n}^{\text {TH }}(F)$ & & $\sigma_{n}^{S H}(F)$ & & $0, n$ & & & $B, n$ & & $B_{2, n}$ & & B...etc. & & \\
\hline+ & & & + & + & + & & & + & & + & & $1+1$ & & & + & & $H$ & & + & & + & 1 \\
\hline+ & & + & & & + & & + & & + & & + & & + & +1 & & $10+$ & +1 & 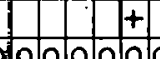 & & + & & +0 \\
\hline
\end{tabular}

Group

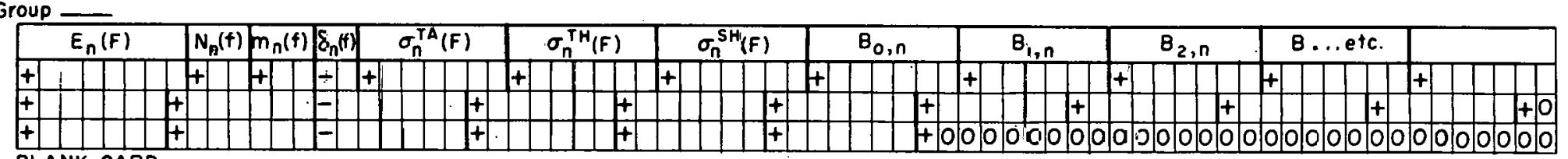
BLANK CARO

Group

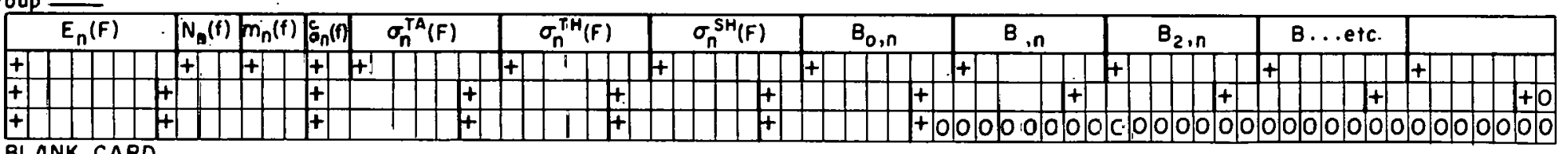
BLANK CARO

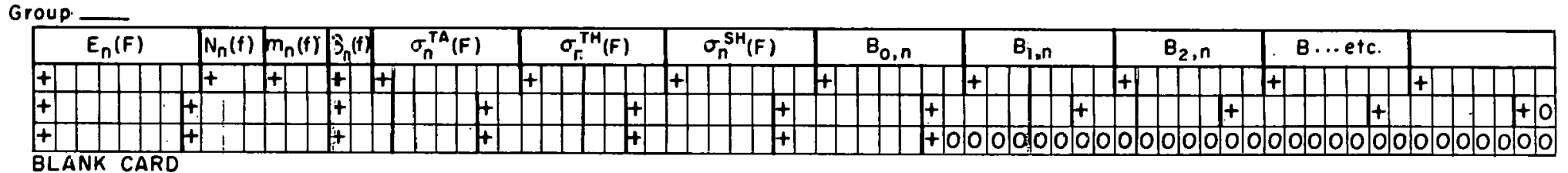

Group

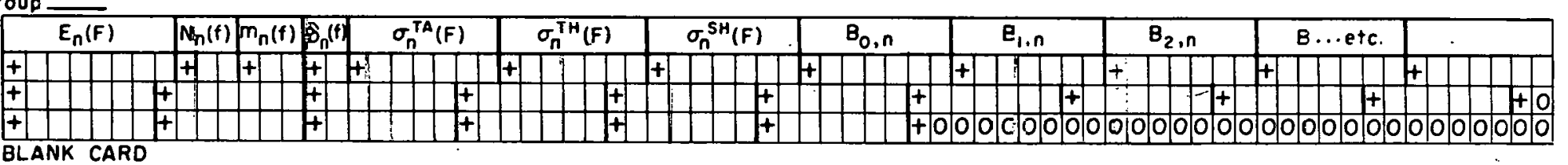




\section{ACKNOWLEDGMENT}

The authors would like to express their appreciation to Dr. Robert C. Gast for many suggestions adopted in the formulation of the code, to Mrs. Carolyn P. Ott for programming the input and recursion routines in EURIPUS -1 and -2 , and to Mrs. Nancy H. Maclay for programming the source preparation and cross section library routines. 